\title{
Constrained optimization framework for interface-aware sub-scale dynamics closure model for multimaterial cells in Lagrangian and arbitrary Lagrangian-Eulerian hydrodynamics
}

\author{
Andrew Barlow ${ }^{a}$, Ryan Hill ${ }^{\text {b,a }}$ Mikhail Shashkov ${ }^{\mathrm{b}, *}$ \\ ${ }^{a}$ Computational Physics Group, AWE Aldermaston \\ Reading, Berkshire, RG7 $4 \mathrm{PR}, \mathrm{UK}$ \\ ${ }^{\mathrm{b}} \mathrm{X}$-Computational Physics, XCP-4 \\ Los Alamos National Laboratory, Los Alamos, NM 87545, USA
}

\begin{abstract}
A systematic description of the new interface-aware sub-scale-dynamics (IA-SSD) closure model for the Lagrangian stage of multimaterial arbitrary Lagrangian-Eulerian methods is presented. The IA-SSD closure model consists of two stages. During the first, bulk, stage, the well known equal compressibility model is used. During the second stage, sub-scale interactions of the materials inside the multimaterial cell are taken into account. At this stage, information about the topology of the materials inside the multimaterial cell is utilized, allowing the orientations of internal interfaces to be included in the model. Each material interacts in a pair-wise fashion with the materials with which it has a common boundary. The interactions are based on the solution of the acoustic Riemann problem between each pair of materials and is limited using physically justified constraints: positivity of volume, positivity of internal energy and controlled rate of pressure relaxation. To determine the values of the limiter coefficients, a constrained-optimization framework is employed using a quadratic objective function with linear constraints. The algorithm guarantees the positivity of the material volume and internal energy as well as the smooth relaxation of the pressure - this allows a significant increase in the robustness of the overall algorithm.

The results of comprehensive testing of the new model have been presented for one- and two-dimensional multimaterial Lagrangian hydrodynamics along with representative results for 2D multimaterial arbitrary Lagrangian-Eulerian (ALE) calculations. The numerical tests have shown that in most cases the new IA-SSD closure model produces better results compared to the well known Tipton's closure model.
\end{abstract}

Key words: Lagrangian hydrodynamics, multimaterial cells, interface-aware sub-scale closure models 


\section{Introduction and rationale}

In the numerical simulations of fluid flows, the choice of computational mesh is crucial. Traditionally, there have been two viewpoints utilizing the Lagrangian or the Eulerian framework, each with its own advantages and disadvantages. In a pioneering paper [1], Hirt et al. developed the formalism for a mesh whose motion could be determined as an independent degree of freedom, and showed that this general framework could be used to combine the best properties of the Lagrangian and Eulerian methods. This class of methods has been termed arbitrary Lagrangian-Eulerian, or ALE.

There are two classes of ALE methods. In the first class, referred to as direct ALE methods, the hydrodynamics equations are solved in an arbitrarily moving coordinate frame, which leads to the appearance of advective terms in the governing equations, [2]. In this paper, only the second class of methods, [1], are considered. In this class the solution process is separated into three distinct stages. These are: 1) a Lagrangian stage, in which the solution and the computational mesh are updated; 2) a rezoning stage, in which the nodes of the computational mesh are moved to more optimal positions; and 3) a remapping stage, in which the Lagrangian solution is conservatively transferred to the rezoned mesh.

Multimaterial cells, which may contain several materials, can appear in Lagrangian calculations. This may occur if the initial configuration consists of several materials, and these materials have complicated geometric shapes, such that it is hard, or impossible, for a given mesh resolution to create an initial mesh which conforms to the material interfaces.

In many multimaterial problems, the initial mesh can be aligned with a material interface. That is, each cell of the mesh contains only one material. For simple flows, it is possible to keep the mesh aligned with the material interfaces so that the nodes on the interfaces do not move, or only move along the interface during the ALE rezoning stage. However, for high-speed multimaterial flows with strong shear deformations it is impossible to keep material interfaces aligned with the mesh. ALE methods are therefore currently the only proven technology to solve such problems. In ALE methods, the mesh does not move with the fluid, and so it is unavoidable that multimaterial cells containing two-or-more materials will appear.

\footnotetext{
* Corresponding author

Email address: shashkov@lanl.gov (Mikhail Shashkov).
} 
Multimaterial cells in ALE methods represent material interfaces that can undergo high deformation. The main research problems concern accurately updating the thermodynamic states of the individual material components in the multimaterial cell, and determining the nodal forces that such a zone generates - despite the lack of information about the velocity distribution within multimaterial cells. A separate set of material properties is normally maintained for all the materials in each multimaterial cell along with the volume fractions that define the proportion of the cell's volume occupied by each material. A closure model is then required to close the governing equations, which are otherwise underdetermined, that is, to define how the volume fractions and states of the individual materials evolve during the Lagrangian step, [3-21]. Most of the traditional closure models do not require knowledge of the positions of the interfaces inside the multimaterial cells, however, more modern methods, like those presented in [18-20,22], do require interface locations and orientations, and therefore require some representation of the interface, [23-25].

The main goal of this paper is to describe the new interface-aware constrainedoptimization based closure model for multimaterial cells during the Lagrangian stage of ALE calculations.

The discretization of Lagrangian hydrodynamics, which is used in this paper, is based on a staggered discretization and principles of compatible discretizations [26]. It is assumed that all materials have the same velocity, but each material has its own density, internal energy and pressure. In the staggered discretization, velocity is defined at the nodes of the mesh. In the single material case, mass, density, internal energy and pressure are defined in the cells of the mesh. In the case of multimaterial flows, there may be pure cells which contain only one material, and multimaterial cells containing several materials. In either case, each cell must produce forces to its nodes. In the case of a pure cell, this force is computed from the pressure of the material in the cell. Forces for multimaterial cells are calculated from an average of all the material component pressures. This average cell pressure is supplied by the closure model and draws upon information from all the materials in the cell.

The Lagrangian stage starts with an update of the nodal velocities as a result of applying zonal forces computed from the material pressure in pure cells, or common (averaged) pressure in multimaterial cells. The updated velocities allow the update of the volumes of all computational cells. For pure cells, the density of the material may then be updated (during the Lagrangian stage, the mass of the material does not change) along with the internal energy of the material. For multimaterial cells, the situation is more complicated. Firstly, the change in the total volume of the multimaterial cell needs to be distributed between materials according to some chosen scheme (which then allows material densities to be updated). Secondly, the update of internal energy for each 
material must be determined. And finally, the common pressure is defined, which is then used in the computation of the forces participating in the momentum equation on the next time step. All these issues are resolved by an appropriate closure model.

The discretization at the Lagrangian stage is required to conserve total momentum and total energy. This imposes additional requirements on the closure model.

The simplest closure model available is the equal compressibility model, [3](Section 3.11), or constant volume fraction model in the case of fluids, where volume fractions are constant in time (during the Lagrangian stage). In this case, the total change in volume of the multimaterial cell is distributed between materials according to a constant in time volume fraction. This model does not take into account any sub-scale interaction between materials inside a multimaterial cell. This model plays an important role in the IA-SSD closure model as the initial, bulk, stage - see Section 3.

One of the important classes of closure models is based on the assumption of pressure equilibrium (PE), or on introducing some mechanism for pressure relaxation $(\mathrm{PR})$ (see, e.g. $[27,4,7,17,13])$. The pressure at a material interface should be continuous; however, the pressure within a computational cell represents an average pressure integrated over the cell volume. This means that there is no physical requirement for absolute pressure equilibration within a multimaterial cell. In fact, for the entire computational cell to come to pressure equilibration, a shock wave would need to cross the cell many times, while the CFL stability condition prohibits a shock wave from crossing any cell in a single time step. However, pressure continuity at material interfaces does suggest that the pressure within a multimaterial cell should move towards equilibration, rather than diverge from it. This can be achieved by introducing a relaxation mechanism like viscosity into the model (see, e.g. [4,13]). In addition to $\mathrm{PE}$ or PR, this class of method invokes conservation of volume and some form of conservation of total internal energy, which is still insufficient to close the model. There are several possibilities for this, e.g. methods like [4] assume that the flow is isentropic. Another model, in which the change in entropy of each material is assumed equal, is proposed in [17] and has the important property that it leads to a hyperbolic system of equations that satisfy an entropy inequality under CFL-like restrictions. Methods in this class also differ with respect to how the equations are approximated, ranging from fully implicit as in $[17,13]$, to fully explicit as in $[4]$. It is important to note that in this class of methods, no knowledge of the actual configuration of materials in the cell is assumed.

One of the PR closure models, which is widely used in production multimaterial arbitrary Lagrangian-Eulerian codes, is the so-called Tipton's model, [4]. 
Due to the importance of this model, and lack of description of the model in the readily accessible literature, a description of how this model is understood and implemented is given in [28]. The results obtained from this model are used as reference solutions in Section 8 where numerical results are presented.

Recently, a new class of closure models that attempt to emulate the behavior of separate Lagrangian sub-cells has been developed - $[16,15,14]$. To the best of our knowledge, the main conceptual ideas of this approach have been presented in [16]. The ideas presented include the pairwise interactions between materials, use of the interface geometry obtained by some form of interface reconstruction, and a simplified acoustic Riemann solver to determine how the interface will move. In schemes $[15,14]$, the position and orientation of the material interface (e.g. from interface reconstruction) is not known. Internal energy is updated separately for each material from its own internal energy equation. A common (averaged) pressure for a multimaterial cell, which is used in the momentum equation, is computed using the principle of conservation of total energy. An exchange of internal energy between the materials inside a multimaterial cell may also be introduced, which allows more freedom in the definition of the common pressure. In [16] a simplified interface reconstruction scheme is used and volume fractions are updated during the Lagrangian step due to sub-cell dynamics (only either expansion or compression is allowed for all the materials in any given time step). The internal energy of each material is then evolved via its own discrete internal energy equation, however this equation differs from the form used in current paper and constraints are not applied to guarantee the positivity of the internal energy and smooth relaxation of material pressures.

Ideas presented in $[16,15,14]$, and also analysis of [4], naturally leads to the idea of a two stage closure model. During the first, bulk, stage, the total change of the volume of the multimaterial cell is distributed to materials using bulk properties of the materials, for example, volume fractions. During the second, sub-scale stage, sub-scale interactions between the materials inside the multimaterial cell are taken into account. The evolution of the material states during the Lagrangian time step is then a combination of the contributions from the bulk and sub-scale stages. For example, a multimaterial cell as a whole may be expanding, therefore during the bulk stage all material volumes in the cell will expand, however due to differences in the material pressures, some materials may compress during the sub-scale stage. The combined update may then lead to an overall compression or expansion of materials.

Following ideas presented in [16], the first step in the development of our new interface-aware sub-scale dynamics (IA-SSD) closure model presented in this paper was performed in [22], where the authors developed a new model which uses information about interfaces between different materials inside a multimaterial cell. The interface geometry is defined by the interface recon- 
struction algorithm. Knowledge of this interface geometry enables the accurate computation the interactions of the materials in a pairwise fashion. In [22], the authors use an acoustic Riemann solution to compute the relative normal velocity for a pair of materials, which allows the computation of the maximum allowable sub-scale volume exchange between each pair of materials. It is clear that there are situations when this maximum allowable volume exchange will need to be limited. For example, consider a two-material case where the first material has a very high pressure and a very large volume fraction ${ }^{1}$. In this situation the maximum allowable sub-scale volume change will lead to a negative volume of the second material, and the volume of the first material will be larger than the volume of the entire multimaterial cell. In [22], in order to prevent a nonphysically large change in material volume, the authors have used a flux-corrected transport (FCT),[29], approach to limit the sub-scale driven part of the volume exchange between materials. The goal was to find limiters which are as close as possible to unity, but still ensure material volumes remain in physically justified bounds. The FCT approach is an approximate way to solve the constrained optimization problem. This model was implemented in the production code 'FLAG' [30].

The next step in the development of our IA-SSD closure model was commenced in reports and conference papers [18-20]. First, it was recognized that the use of FCT to compute the limiters can lead to locking situations ${ }^{2}$ when more than two materials are present in a cell. To avoid this issue, the FCT approach was replaced by a fully quadratic optimization problem with linear constraints, [31,32]. Secondly, two additional constraints were introduced: one related to the positivity of internal energy of each material component, and another related to controlling pressure equilibration. Both of these constraints can be formulated in an approximate form as linear constraints with respect to the volume exchange. It may be noted that the pressure relaxation constraint appears to be the most significant constraint, especially in the case where very small volume fractions are present, and in most cases observed to date, drives the definition of the limiter.

In this paper, a systematic and comprehensive description of the new IA-SSD closure model introduced in [18-20] is given. A new procedure for updating material centroid positions, which is needed for the moment-of-fluid interface reconstruction algorithm, [23-25] is also presented. The new material centroid update algorithm is closely connected to the IA-SSD closure model.

Results of comprehensive testing of the new model for one- and two-dimensional

$\overline{1}$ Here it is necessary to emphasize that the volume exchange is proportional to the time step, which is defined using averaged values in multimaterial cells and does not depend on volume fractions, that is, the time step can be quite large.

2 That is, pressure in the multimaterial cell will not equilibrate, because in the FCT approach, the most cautious case is adopted. 
multimaterial Lagrangian hydrodynamics are presented along with two-dimensional multimaterial arbitrary Lagrangian-Eulerian calculations.

Readers who are interested in other possible approaches for closure models are referred to the following journal papers, Section 3.11 of [3], Sections 6.2 and 6.3 of [33] and [34,6,7,35-43]. Also of note is [36], where the author considers a thermodynamic and dynamic subgrid closure model for a two-material cell, and the recent paper by B. Rider and co-authors, which discusses the use of limiters to combine a Tipton-like model and the equal compressibility model, [44].

The remainder of the paper is organized as follows. Section 2 presents a discussion covering different forms of the internal energy equation, which will be needed for the derivation of the IA-SSD closure model. In Section 3 the simplest model of equal compressibility is described. This model represents the bulk stage of the IA-SSD algorithm. The sub-scale stage of the new closure model is described in Section 4. In Section 5 we explain how our new closure model is incorporated in compatible staggered discretization for multimaterial Lagrangian hydrodynamics. The summary of the closure model algorithm is given in Section 6. The time evolution algorithm of the material centroids is described in Section 7. Numerical examples are given in Section 8. In this section results obtained by the new IA-SSD model are presented and compared with those obtained by Tipton's model. Conclusions and a description of future research is provided in Section 9.

\section{Lagrangian hydrodynamics. Different forms of the internal en- ergy equation}

The basic assumption of all Lagrangian algorithms is that there exists a discrete volume element, $V$, that may deform in shape but through whose boundary no mass flows. Thus the original mass, $m$, present in the volume at some starting time is constant; $d m / d t=0$, here $d / d t$ is the total time derivative following the fluid element. At any later time, $t$, the density, $\rho$, inside the given element is found from $\rho=m / V(t)$. Substituting this expression into the usual continuity equation for density results in the statement that

$$
\frac{1}{V} \frac{d V}{d t}=\operatorname{div} \mathbf{u}
$$

where $\mathbf{u}$ denotes the velocity.

Next, consider the equation of motion with the force given as the gradient of a scalar pressure $p$, and also, the associated equation for the evolution of the 
specific internal energy $\varepsilon$. Written in semi-Lagrangian form these equations are

$$
\begin{aligned}
& \rho \frac{d u}{d t}=-\operatorname{grad} p \\
& \rho \frac{d \varepsilon}{d t}=-p \operatorname{div} \mathbf{u} .
\end{aligned}
$$

Then, conservation of total energy can be obtained from the above equations simply by multiplying Eq. (2) by the velocity u, adding the result to Eq. (3), and integrating over some domain $D$ in which these equations are defined. This gives the result

$$
\int_{D} \rho\left(\frac{d\left(|\mathbf{u}|^{2} / 2\right)}{d t}+\frac{d \varepsilon}{d t}\right) d V=-\int_{D}(\mathbf{u} \cdot \operatorname{grad} p+p \operatorname{divu}) d V=-\oint_{\partial D} p \mathbf{u} \cdot \mathbf{d} \mathbf{S} .(4)
$$

That is, conservation of total energy follows from this property of the divergence and gradient operators

$$
\int_{D}(\mathbf{u} \cdot \operatorname{grad} p+p \operatorname{div} \mathbf{u}) d V=\oint_{\partial D} p \mathbf{u} \cdot \mathbf{d} \mathbf{S} .
$$

This shows the importance of consistent and compatible discretizations of the momentum and internal energy equations such that the discrete analog of identity (5) has to be satisfied in the discrete case.

Due to (1), the internal energy equation can be expressed in the isentropic form (which we will sometimes call $p d V$ form of the internal energy equation),

$$
\rho \frac{d \varepsilon}{d t}=-p \frac{1}{V} \frac{d V}{d t}
$$

which is more physical as it demonstrates that entropy cannot be reduced by the scheme.

Taking into account the definition of the Lagrangian mass, equation (6) can also be written as

$$
m d \varepsilon=-p d V .
$$

In the continuum case both forms of energy equation $((7)$ and (3)) are equivalent. However, in the discrete case they are not. In this paper, both forms of the energy equation are required. Equation (7) is used in the derivation of the closure model for multimaterial cells. Equation (3) is used in the derivation of the compatible discretization, which conserves total energy, 


\section{Equal compressibility closure model for the multimaterial case}

\subsection{Motivation}

In the multimaterial case, multimaterial zones are identified by the presence of more than one material. The index $i$ is used to identify specific materials. The set of materials in zone $z$ is denoted by $M(z)$. The volume of material $i$ in zone $z$ at time $t^{n}$ is denoted by $V_{z, i}^{n}$. Each material has its own mass, $m_{z, i}$; density $\rho_{z, i}^{n}$; pressure, $p_{z, i}^{n} ;$ and internal energy, $\varepsilon_{z, i}^{n}$. It is useful to introduce the volume fraction for each material, $0<\alpha_{z, i}^{n}<1$. Note that the Lagrangian calculation is considered here, therefore if a material is present in the multimaterial cell at time $t^{n}$, it will remain in the cell upon the completion of the Lagrangian step. When dealing with only one zone, the zonal subscript $z$ is dropped from the notation.

The simplest closure model is the equal compressibility model, whereby it is assumed that the volume fraction does not change in time, i.e. $\alpha_{z, i}^{n+1}=\alpha_{z, i}^{n}$. When applied to solids, this model is called the equal volumetric strain model.

For any discretization considered in this paper, the time step starts with the computation of the new nodal velocities. To discretize the momentum equation, a single pressure per zone is required - even in the case of multimaterial zones. This pressure is referred to as the average pressure. The computation of this pressure is performed by the closure model and will be described later in this section. For now, it may be assumed that this average pressure is given. The new nodal velocities enable the new cell volumes $V_{z}^{n+1}$ at $t^{n+1}$. Therefore, the total change in the volume of any zone, $\Delta V_{z}^{n+1}=V_{z}^{n+1}-V_{z}^{n}$ is also known.

If a zone contains multiple materials, the equal compressibility closure model distributes $\Delta V_{z}^{n+1}$ between materials as follows

$$
\Delta V_{z, i}^{n+1}=\alpha_{z, i}^{n} \Delta V_{z}^{n+1}
$$

that is, the total change in the volume of the multimaterial cell is distributed between materials proportionally to the material volume fraction from the previous time step.

The corresponding material volumes at $t^{n+1}$ are

$$
V_{z, i}^{n+1}=V_{z, i}^{n}+\Delta V_{z, i}^{n+1}=\alpha_{z, i}^{n}\left(V_{z}^{n}+\Delta V_{z}^{n+1}\right)=\alpha_{z, i}^{n} V_{z}^{n+1} .
$$

Assuming that the mesh at time $t^{n+1}$ is not tangled, $\left(V_{z}^{n+1}>0\right)$, then the material volumes at $t^{n+1}$ will also be positive, $V_{z, i}^{n+1}>0$. This fact is crucial 
in the construction of the sub-scale closure model.

The material densities may then be updated as

$$
\rho_{z, i}^{n+1}=m_{z, i} / V_{z, i}^{n+1}
$$

The update of internal energies for each material is done using individual $p d V$ equations and expression (8) for change in the material volume

$$
m_{z, i}\left(\varepsilon_{z, i}^{n+1}-\varepsilon_{z, i}^{n}\right)=-p_{z, i}^{n} \Delta V_{z, i}^{n+1}=-p_{z, i}^{n} \alpha_{z, i}^{n} \Delta V_{z}^{n+1} .
$$

The total internal energy for material $i$ in zone $z$ is

$$
\mathcal{E}_{z, i}^{n}=m_{z, i} \varepsilon_{z, i}^{n},
$$

and the total internal energy in the zone $z$ is

$$
\mathcal{E}_{z}^{n}=\sum_{i \in M(z)} \mathcal{E}_{z, i}^{n}
$$

The specific internal energy in the multimaterial cell can be defined as

$$
\varepsilon_{z}^{n}=\frac{\mathcal{E}_{z}^{n}}{m_{z}}=\frac{\sum_{i \in M(z)} \mathcal{E}_{z, i}^{n}}{\sum_{i \in M(z)} m_{z, i}} .
$$

Summing equations (11) in zone $z$ over all materials, the total change of internal energy in the multimaterial cell is obtained as

$$
\begin{aligned}
& m_{z}\left(\varepsilon_{z}^{n+1}-\varepsilon_{z}^{n}\right)=\sum_{i \in M(z)}\left[m_{z, i}\left(\varepsilon_{z, i}^{n+1}-\varepsilon_{z, i}^{n}\right)\right]= \\
& -\sum_{i \in M(z)}\left[p_{z, i}^{n} \Delta V_{z, i}^{n+1}\right]=-\sum_{i \in M(z)}\left[p_{z, i}^{n} \alpha_{z, i}^{n} \Delta V_{z}^{n+1}\right] \\
& =-\left\{\sum_{i \in M(z)}\left[p_{z, i}^{n} \alpha_{z, i}^{n}\right]\right\} \Delta V_{z}^{n+1} .
\end{aligned}
$$

If the average zonal pressure $\tilde{p}_{z}^{n}$ is defined as

$$
\tilde{p}_{z}^{n}=\sum_{i \in M(z)}\left[\alpha_{z, i}^{n} p_{z, i}^{n}\right]
$$


then equation (15) may be written in a form resembling the internal energy equation, (7), for the single material case if $p_{z}^{n}$ is replaced by $\tilde{p}_{z}^{n}$

$$
m_{z}\left(\varepsilon_{z}^{n+1}-\varepsilon_{z}^{n}\right)=-\tilde{p}_{z}^{n} \Delta V_{z}^{n+1}
$$

This observation and logic of compatible discretization, [26] (see [28] for details), suggests that for a multimaterial zone, the average pressure in the discretization of the momentum equation needs to be taken as $\tilde{p}_{z}^{n}$.

\subsection{Artificial viscosity}

There are two approaches to deal with artificial viscosity for multimaterial zones.

Distribution approach. This approach is based on first computing viscosity forces for the entire multimaterial zone by using averaged quantities. For example, averaged density as total mass divided by total volume, and other formulas for averaged speed of sound, etc. Total heat from artificial viscosity forces may then be calculated as described in [45] for the single material case (see also equation (97). The total heat from artificial viscosity forces may then be distributed between materials in the multimaterial zone. Many schemes are available to do this. The simplest method is to distribute it to all materials proportionally by mass fraction as done in this paper. Another option is to distribute it only to the materials which are under compression, and again use mass fraction but only with respect to materials under compression (i.e. the ratio of mass of the material to the sum of masses of the materials which are under compression). This approach works for any type of viscosity which is used for pure zones.

Gathering approach. In this case, an artificial viscosity is computed for each material. The material viscosities are then used to produce a zonal viscosity force which participates in the momentum equation. Each material viscosity produces heating which is used in the individual internal energy equations. The use of this approach has been trialled in the case of bulk material viscosity, which only uses change in material volume. This approach requires more investigation, and results are not reported in this paper. Furthermore, it is not clear how to use this approach for more complicated formulations of artificial viscosity.

The question regarding the best approach to deal with artificial viscosity for multimaterial cells is an area of active research. Reporting of the findings of this research is planned for future papers. 
3.3 Time step control: multimaterial considerations - equal compressibility model

There are two main constraints on the time step. The first constraint is related to the Courant stability condition, usually written as

$$
\Delta t \leq \Delta t_{\text {courant }}=\min _{z} \frac{L_{z}}{\tilde{c}_{z}}
$$

where $L_{z}$ is some characteristic length associated with zone $z$ and $\tilde{c}_{z}$ is some effective zonal speed of sound, which also includes information about artificial viscosity - see, for example, Section 4 in [26].

For the equal compressibility model used at the bulk stage, the expression for the adiabatic speed of sound is

$$
\rho_{z} c_{z}^{2}=\sum_{i \in M(z)}\left(\alpha_{z, i} \rho_{z, i} c_{z, i}^{2}\right)
$$

This, in particular, means that the speed of sound for the multimaterial cell lies between the maximum and minimum values of the speed of sound for all materials in the multimaterial cell.

Another constraint on time step relates to the positivity of internal energy. In the equal compressibility closure model, the internal energy equation for each material is

$$
m_{i}\left(\varepsilon_{i}^{n+1}-\varepsilon_{i}^{n}\right)=-p_{i}^{n} \Delta V_{i}^{n+1}+Q_{i}^{n} .
$$

The heating $Q_{i}^{n}$ from artificial viscosity is positive by construction, therefore, for $\varepsilon_{i}^{n+1}$ defined from this equation to be positive, it is enough to choose $\Delta t$ such that

$$
m_{i} \varepsilon_{i}^{n}-p_{i}^{n} \Delta V_{i}^{n+1}>0
$$

It is assumed that the internal energy on the previous time step is positive, $\varepsilon_{i}^{n}>0$.

If $\Delta V^{n+1} \leq 0$, so that the entire cell is under compression, then $\Delta V_{i}^{n+1}=$ $\alpha_{i}^{n} \Delta V^{n+1}$ is negative, and therefore there is no additional requirement on $\Delta t$ to satisfy (21), because the summation involves the addition of two positive numbers. 
If $\Delta V^{n+1}>0$ then inequality (21) means that

$$
m_{i} \varepsilon_{i}^{n}>p_{i}^{n} \Delta V_{i}^{n+1}
$$

or

$$
\Delta V^{n+1}<\frac{m_{i} \varepsilon_{i}^{n}}{p_{i}^{n} \alpha_{i}^{n}}
$$

Because $m_{i}=\rho_{i}^{n} V_{i}^{n}=\rho_{i}^{n} \alpha_{i}^{n} V^{n}$ inequality (23) leads to the following inequality

$$
\Delta V^{n+1}<\frac{\rho_{i}^{n} V^{n} \varepsilon_{i}^{n}}{p_{i}^{n}}
$$

or

$$
\frac{\Delta V^{n+1}}{V^{n}}<\frac{\rho_{i}^{n} \varepsilon_{i}^{n}}{p_{i}^{n}}
$$

Because DIVu $=\frac{1}{V} \frac{d V}{d t}$, inequality (25) can be rewritten as

$$
\Delta t<\frac{\rho_{i}^{n} \varepsilon_{i}^{n}}{p_{i}^{n}} \cdot \frac{1}{\mathbf{D I V u}}
$$

The most important consequence of this inequality is that this constraint on $\Delta t$ does not depend on volume fractions and indeed the corresponding constraint is true for pure cell containing only material $i$.

The inequality (26) is true for any equation of state, for ideal gas $p_{i}^{n}=\left(\gamma_{i}-\right.$ 1) $\rho_{i}^{n} \varepsilon_{i}^{n}$, and the constraints are

$$
\Delta t<\frac{1}{\left(\gamma_{i}-1\right) \mathbf{D I V u}}
$$

\section{Sub-scale model}

\subsection{Motivation}

In this section, where it does not lead to misunderstanding, the zonal index $z$ is dropped because only a single zone is considered. Additionally, in most cases the time index is also dropped. 


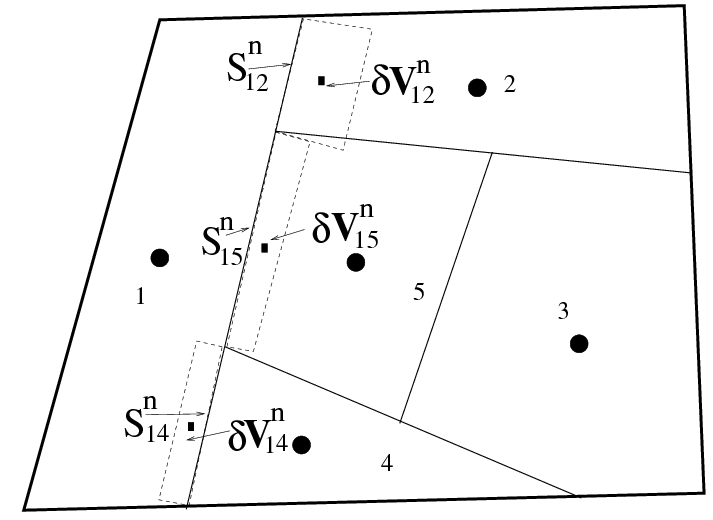

Fig. 1. Multimaterial cell. Representation of the materials inside the multimaterial cell with the definition of the relative volume exchange between materials and time advance of centroids.

It is assumed that the total change of volume for the multimaterial zone, $\Delta V$, is given. It is also assumed that the topology of the materials inside multimaterial zone is known from the interface reconstruction algorithm. Each material is represented by a pure sub-polygon, and for each material $i$ the set of the materials, $k \in M(i)$, which have a common edge with material $i$ is known. The area of the common edge is denoted by $S_{i, k}$, and the unit normal to the common edge pointing from material $i$ to material $k$ is denoted by $\mathbf{n}_{i, k}$. The graphical illustration is given in Fig. 1.

In deriving the sub-scale model, the logic used in the previous section is followed, i.e. starting with the model for material volume change, followed by the derivation of the equation for material internal energy.

\section{Volume change model}

The volume change of material $i$ described in the previous Section is now denoted

$$
\Delta V_{i}^{\text {bulk }}=\alpha_{i} \Delta V
$$

with the corresponding value of the material volume denoted as

$$
V_{i}^{\text {bulk }}=\alpha_{i} V^{n+1}
$$

This change of volume is referred to as bulk because it is the result of the distribution of the change of volume of the entire multimaterial cell, which does not take into account sub-scale dynamics.

To take into account the difference in pressure of the materials inside the multimaterial zone, the volume exchange between neighboring materials in 
flux form is introduced as

$$
\Delta V_{i}=\Delta V_{i}^{\text {bulk }}+\sum_{k \in M(i)} \delta V_{i, k}, \quad \delta V_{i, k}=-\delta V_{k, i},
$$

where the explicit form of $\delta V_{i, k}$ will be described later.

This form conserves the total change of the zonal volume

$$
\sum_{i \in M(z)} \Delta V_{i}=\Delta V
$$

because $\sum_{i \in M(z)} \Delta V_{i}^{\text {bulk }}=\Delta V$ and because the sub-scale exchange term is in "flux" form.

The volume exchange term is constructed in the form

$$
\delta V_{i, k}=\Psi_{i, k} \delta V_{i, k}^{\max }
$$

where $\delta V_{i, k}^{\max }$ is defined from some physical considerations taking into account material interaction, and $0 \leq \Psi_{i, k} \leq 1$ are limiters which are chosen in such a way that the overall model does not violate physically justified constraints - for example, the positivity of material volumes. The obvious requirement $0 \leq \Psi_{i, k}$ ensures that the limiter does not reverse the direction of the volume change acting against the pressure difference between the materials, which would be non-physical. The definition of the upper bound $\Psi_{i, k} \leq 1$ is more questionable, however, it is sensible to not increase the magnitude of the sub-scale fluxes.

The value of $\delta V_{i, k}^{\max }$ is estimated using the acoustic Riemann solver

$$
\delta V_{i, k}^{\max }=\frac{p_{i}-p_{k}}{\rho_{i} c_{i}+\rho_{k} c_{k}} S_{i, k} \Delta t
$$

where $c_{i}$ is the adiabatic speed of sound of material $i$, and $S_{i, k}$ is the area of interface between materials $i$ and $k$, see Fig. ${ }^{3}$.

In Fig.1 a multimaterial cell with five materials is presented with a graphical representation of $\delta V_{i, k}$ (the same notation is used for its volume and the corresponding rectangle). For material \#1, $(i=1)$; there are three neighbors:

3 For the sake of brevity, the time discretization for quantities in equation (33) are not specified. For example, values at $t^{n}$, or estimated values after the bulk update may be used. The time discretization is presented in the description of the complete algorithm. 
$\{2,4,5\}$. In the situation presented in Fig. $1 p_{1}$ is larger than $p_{2}$ and $p_{5}$, and smaller than $p_{4}$.

It can be noted that in principle, a more accurate expression for $\delta V_{i, k}^{\max }$ may be derived compared to the form given by Eq. (33). For example, in a recently published paper [36], the author derives an implicit analytical solution for the two material case - p. 137 in [36]. Future work is planned to use the results presented in [36] and extend it to the case of three or more materials.

\section{Bounds for material volume}

It is necessary that the volume of each material is larger than zero and less than the zonal volume

$$
V^{n+1}>V_{i}^{n+1}>0
$$

However, there are some considerations here. Clearly, volume may not reduce exactly to zero because the mass of the material cannot disappear in the Lagrangian step, and if the volume of any material is zero, then its density will be infinity. Also, constraints need to be imposed which can be achieved for the chosen form of volume change for each material for some value of limiters.

Therefore, the following constraints are imposed:

$$
V_{i}^{n+1} \geq \kappa_{\text {bot }} V_{i}^{n+1, \text { bulk }}, 1 \geq \kappa_{\text {bot }}>0 .
$$

Here, the parameter $\kappa_{b o t}$ controls how close to zero the volume of the material may reach. In principle, $\kappa_{\text {bot }}$ may be different for each zone, material in the zone, or be dependent upon time. The constraint on volume is intended to ensure the volume stays positive, finite and the density of the material does not exceed the maximum density that the material can be compressed to. In results presented in this paper constant in time value of $\kappa_{b o t}$ is used for all materials.

The proof that constraint (35) is sufficient to guarantee that requirements (34) are satisfied and that constraint (35) is in the feasible set is shown below. Specifically, the constraint may be satisfied by choosing all $\Psi_{z ; i, k}$ equal to zero.

Because it is assumed that $\alpha_{i}^{n}>0, V^{n+1}>0$ and $\kappa_{\text {bot }}>0$ inequality (35) implies that $V_{i}^{n+1}>0$. Therefore the inequality on the right of (34) is satisfied.

Now, because for each zone

$$
\sum_{i} V_{i}^{n+1}=V^{n+1}
$$


then for an arbitrary $i_{0}$,

$$
V_{i_{0}}^{n+1}=V^{n+1}-\sum_{i ; i \neq i_{0}} V_{i}^{n+1}
$$

Now, because from (35) $V_{i}^{n+1}>0$, equation (37) implies that $V_{i_{0}}^{n+1}<V^{n+1}$. Therefore the inequality on the left of (34) is satisfied. Inequality (35) is therefore sufficient to satisfy inequality (34).

Indeed, a more precise estimate is available. Using (37) and (35) it can be shown that

$$
\begin{aligned}
V_{i_{0}}^{n+1} \leq V^{n+1}-\sum_{i \neq i_{0}}\left(\kappa_{b o t} V^{n+1} \alpha_{i}^{n}\right) & =V^{n+1}\left(1-\kappa_{b o t} \sum_{i \neq i_{0}} \alpha_{i}^{n}\right) \\
& =V^{n+1}\left[1-\kappa_{b o t}\left(1-\alpha_{i_{0}}^{n}\right)\right]
\end{aligned}
$$

where $\left[1-\kappa_{b o t}\left(1-\alpha_{i_{0}}^{n}\right)\right]$ is a positive number.

Choosing the model (30),(32), and (33) for the material volume update, the constraint (35) represents a system of linear inequalities with respect to the limiters $\Psi_{i, k}$

$$
V_{i}^{n+1, b u l k}+\sum_{k \in M(i)} \Psi_{i, k} \delta V_{i, k}^{\max } \geq \kappa_{b o t} V_{i}^{n+1, b u l k} .
$$

Because $1 \geq \kappa_{b o t}$, it is obvious that if all $\Psi_{i, k}$ are zero, then requirements (35) are satisfied. There is therefore at least one solution of this inequality.

\section{Positivity of internal energy}

In the IA-SSD closure model, each material has a separate approximate $p d V$ equation.

The most natural way to write the $p d V$ equation for material $i$ is ${ }^{4}$

$$
m_{i}\left(\varepsilon_{i}^{n+1}-\varepsilon_{i}^{n}\right) \approx-p_{i}^{n} \Delta V_{i}^{n+1}+Q_{z, i}^{n}
$$

Taking into account the above model for the change of the material volume, equation (40) can be rewritten as

$$
m_{i}\left(\varepsilon_{i}^{n+1}-\varepsilon_{i}^{n}\right) \approx-p_{i}^{n} \alpha_{i}^{n} \Delta V^{n+1}-p_{i}^{n} \sum_{k \in M(i)} \Psi_{i, k} \delta V_{i, k}^{\max }+Q_{z, i}^{n} .
$$

$\overline{4}$ To explain the idea, the predictor stage is considered. 
The first term on the RHS, $p_{i}^{n} \alpha_{i}^{n} \Delta V^{n+1, p r}$, corresponds to the equal compressibility model as described in the previous Section, The second term on the RHS, $p_{i}^{n} \sum_{i} \Psi_{i, k} \delta V_{i, k}^{\max }$, is not in flux form, and therefore will lead to a violation of the conservation of total energy. As suggested in, [8], $p_{i}^{n}$ is replaced by the interfacial pressure $p_{i, k}^{*}$ and it is moved under the summation to form a flux exchange of internal energy between materials $i$ and $k$.

$$
m_{i}\left(\varepsilon_{i}^{n+1}-\varepsilon_{i}^{n}\right)=-p_{i}^{n} \alpha_{i}^{n} \Delta V^{n+1}-\sum_{k \in M(i)} p_{i, k}^{*} \Psi_{i, k} \delta V_{i, k}^{\max }+Q_{z, i}^{n} .
$$

The pressure $p_{i, k}^{*}$ is obtained from the one-dimensional acoustic Riemann problem between materials $i$ and $k^{5}$ :

$$
p_{i, k}^{*}=\frac{\left(\rho_{k} c_{k}\right) p_{i}+\left(\rho_{i} c_{i}\right) p_{k}-\left(\rho_{k} c_{k}\right)\left(\rho_{i} c_{i}\right)\left(\mathbf{u}_{k}-\mathbf{u}_{i}\right) \cdot \mathbf{n}_{i, k}}{\rho_{k} c_{k}+\rho_{i} c_{i}} .
$$

It is assumed that $\Delta t$ is chosen in such a way that if all $\Psi_{i, k}$ are zero, then the internal energies of all materials at the new time step are positive, i.e. the time step guarantees positivity of internal energy for each material obtained by the equal compressibility model.

If all $\Psi_{i, k}$ are zero then

$$
m_{i}\left(\varepsilon_{i}^{n+1}-\varepsilon_{i}^{n}\right)=-p_{i}^{n} \Delta V_{i}^{n+1, b u l k}+Q_{z, i}^{n} .
$$

The equal compressibility closure model is then recovered, and it is known that $\Delta t$ can be chosen such that the internal energy of the material is positive.

In what follows it is assumed that $\Delta t$ is chosen such that

$$
\Delta \mathcal{E}_{i}^{\text {bulk }}=m_{i} \varepsilon_{i}^{n}-p_{i}^{n} \Delta V_{i}^{n+1, \text { bulk }}+Q_{i}^{n}>0
$$

Then,

$$
m_{i} \varepsilon_{i}^{n+1}=\Delta \mathcal{E}_{i}^{b u l k}-\sum_{k \in M(i)} \Psi_{i, k} p_{i, k}^{*} \Delta V_{i, k}^{\max }
$$

and the inequality required for the positivity of internal energy on the new

5 The time levels used for the quantities in equation (43) have been intentionally omitted. The choice for the predictor stage is obvious because only data from $t=t^{n}$ is available. However, many possibilities exist for the corrector stage. The chosen time level is presented in the summary of the complete discrete algorithm. 
time step can be written as

$$
\Delta \mathcal{E}_{i}^{b u l k} \geq \sum_{k \in M(i)} \Psi_{i, k} p_{i, k}^{*} \Delta V_{i, k}^{\max }
$$

By assumption, the left-hand side (LHS) of this inequality is positive, therefore if all $\Psi_{i, k}$ are zero this inequality is satisfied.

In general, (46) is a system of linear inequalities with respect to $\Psi_{i, k}$ and it has been shown that there exists at least one solution.

\section{Controlling pressure equilibration}

Another important property of closure models is the manner in which pressure equilibration is achieved in time ${ }^{6}$.

Pressure equilibration should be achieved without oscillations, and in such a way that the pressures of different materials approach equilibrium in a smooth fashion. This is especially important for Lagrangian calculations featuring very small volume fractions for some materials. This is a very common situation in multimaterial calculations if the initial mesh is not aligned with material interfaces, which is usually the case when material interfaces have a complicated geometric shape. Numerical experiments show that smooth pressure relaxation is critical for the stability of the method. For multimaterial ALE calculations, small volume fractions frequently appear because materials are moving through the mesh.

Consider the evolution of material pressure during a single time step. Using an equation of state with the form $p=\mathcal{P}(\rho, S)$, where $\rho$ is density and $S$ is entropy, it follows that

$$
\begin{aligned}
& p^{n+1} \approx p\left(t^{n+1}\right)=\mathcal{P}\left(\rho\left(t^{n+1}\right), S\left(t^{n+1}\right)\right) \approx \\
& p^{n}+\left\{\left.\left.\frac{\partial \mathcal{P}}{\partial \rho}\right|_{t=t^{n}} \frac{\partial \rho}{\partial t}\right|_{t=t^{n}} \Delta t+\left.\left.\frac{\partial \mathcal{P}}{\partial S}\right|_{t=t^{n}} \frac{\partial S}{\partial t}\right|_{t=t^{n}} \Delta t\right\}+(\Delta t)^{2} .
\end{aligned}
$$

First, $\frac{\partial \mathcal{P}}{\partial \rho}=c^{2}$. Next,

$$
\frac{\partial \rho}{\partial t}=\frac{\partial(m / V)}{\partial t}=m \frac{\partial(1 / V)}{\partial t}=-m \frac{1}{V^{2}} \frac{d V}{/ d t}=-\rho V \frac{1}{V^{2}} \frac{d V}{/ d t}=-\rho \frac{1}{V} \frac{d V}{d t},
$$

Taking into account this expression, the first term in braces in (47) can be

\footnotetext{
$\overline{6}$ Here, the situation is considered where there is some transition time after which
} no new forces are applied to the multimaterial cell and contact is established. 
estimated as

$$
\left.\left.\frac{\partial \mathcal{P}}{\partial \rho}\right|_{t=t^{n}} \frac{\partial \rho}{\partial t}\right|_{t=t^{n}} \Delta t \approx-\left.\frac{c^{2} \rho}{V}\right|_{t^{n}} \Delta V^{n+1},
$$

where $\Delta V^{n+1}=V^{n+1}-V^{n}$, that is, the first term in braces in equation (47) is proportional to $\Delta V^{n+1}$.

Now, consider the second term inside the braces in (47). For weak shocks there is a well known result, [50] (p. 64), that the change of entropy is proportional to $(\Delta V)^{3}$, therefore in this case, the second term inside the braces is smaller than the first term and can be ignored ${ }^{7}$. In general, ignoring this term can be considered a modeling assumption, the validity of such assumption can be checked by numerical examples.

Taking into account these considerations, the following approximation for material pressure evolution may be obtained

$$
\begin{aligned}
& \tilde{p}_{i}^{n+1}\left(\Psi_{i, k}\right)=p_{i}^{n}-\rho_{i}^{n}\left(c_{i}^{n}\right)^{2} \Delta V_{i}^{n+1} / V_{i}^{n}= \\
& \tilde{p}_{i}^{b u l k, n+1}-\frac{\rho_{i}^{n}\left(c_{i}^{n}\right)^{2}}{V_{i}^{n}} \sum_{k \in M(i)} \Psi_{i, k} \delta V_{i, k}^{\max }
\end{aligned}
$$

where pressures

$$
\tilde{p}_{i}^{b u l k, n+1}=p_{i}^{n}-\frac{\rho_{i}^{n}\left(c_{i}^{n}\right)^{2}}{V_{i}^{n}} \Delta V_{i}^{b u l k, n+1}
$$

are given, and the second term on the RHS of (50) is again a linear function of $\Psi_{i, k}$.

To achieve smooth equilibration, a temporarily targeted equilibrated pressure, $\bar{p}$, is computed from known quantities as the pressure towards which the material pressures are intended to relax toward at this time moment. The simplest choice is to set $\bar{p}=\sum_{i} \alpha_{i}^{n} \tilde{p}_{i}^{b u l k, n+1}$. Material pressures $p_{i}^{b u l k, n+1}$ may be higher or lower than $\bar{p}$, however, $\max _{i} \tilde{p}_{i}^{b u l k, n+1} \geq \bar{p} \geq \min _{i} \tilde{p}_{i}^{b u l k, n+1}$. The limiters are chosen in such a way that $\tilde{p}_{i}^{n+1}$ defined by equation (50) will relax toward (if possible, or at least not diverge from) $\bar{p}$.

Consider the case when $\tilde{p}_{i}^{b u l k, n+1} \geq \bar{p}$. In this case, it is required that

$$
\kappa_{i} \bar{p}+\left(1-\kappa_{i}\right) \tilde{p}_{i}^{b u l k, n+1} \leq \tilde{p}_{i}^{n+1} \leq \tilde{p}_{i}^{b u l k, n+1} .
$$

Here $0<\kappa_{i}<1$ is a parameter which controls the rate of relaxation. In numerical results presented in this paper $\kappa_{i}=0.5$.

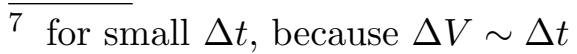


Using (50), inequality (51) transfers to following requirement for $\Psi_{i, k}$

$$
\frac{\kappa_{i} V_{i}^{n}}{\rho_{i}^{n}\left(c_{i}^{n}\right)^{2}}\left(\tilde{p}_{i}^{b u l k, n+1}-\bar{p}\right) \geq \sum_{k \in M(i)} \Psi_{i, k} \delta V_{i, k}^{\max } \geq 0,
$$

which can be satisfied if all $\Psi_{i, k}$ are zero, because by assumption $\left(\tilde{p}_{i}^{b u l k, n+1}-\right.$ $\bar{p}) \geq 0$ and the coefficient in front of this expression on the LHS of the inequality is positive. Therefore, inequality (52) is an additional linear inequality for $\Psi_{i, k}$, which has at least one solution.

Now, consider the case when $\tilde{p}_{i}^{b u l k, n+1} \leq \bar{p}$; then it is required that

$$
\kappa_{i} \bar{p}+\left(1-\kappa_{i}\right) \tilde{p}_{i}^{b u l k, n+1} \geq \tilde{p}_{i}^{n+1} \geq \tilde{p}_{i}^{b u l k, n+1} .
$$

This leads to the following inequality

$$
\frac{\kappa_{i} V_{i}^{n}}{\rho_{i}^{n}\left(c_{i}^{n}\right)^{2}}\left(\tilde{p}_{i}^{b u l k, n+1}-\bar{p}\right) \leq \sum_{k \in M(i)} \Psi_{i, k} \delta V_{i, k}^{\max } \leq 0,
$$

which is satisfied if all $\Psi_{i, k}$ are zero, because by assumption $\left(\tilde{p}_{i}^{b u l k, n+1}-\bar{p}\right) \leq 0$ and the coefficient in front of this expression on the LHS of the inequality is positive. Therefore, inequality (54) is also an additional linear inequality for $\Psi_{i, k}$, which has at least one solution.

\subsection{Constrained optimization framework}

For each multimaterial zone $z$, the following minimization problem is solved

$$
\min _{\Psi_{i, k}}\left\{\sum_{i}\left[\sum_{k \in M(i)}\left(1-\Psi_{i, k}\right)^{2}\right]\right\} .
$$

The linear constraints come from the volume bounds, positivity of internal energy and pressure equilibration conditions described in the previous Section. For each material $i$, the constraints for the limiters $\Psi_{i, k}$ are:

The material volume related constraint, (39);

$$
\sum_{k \in M(i)} \Psi_{i, k} \delta V_{i, k}^{\max } \geq\left(\kappa_{b o t}-1\right) V_{i}^{n+1, b u l k}
$$


The constraint related to positivity of material internal energy, (46);

$$
\left(m_{i} \varepsilon_{i}^{n}-p_{i}^{n} \Delta V_{i}^{n+1, \text { bulk }}+Q_{i}^{n}\right) \geq \sum_{k \in M(i)} \Psi_{i, k} p_{i, k}^{*} \Delta V_{i, k}^{\max }
$$

The constraint related to controlling pressure equilibration; (52), (54)

$$
\begin{aligned}
& \frac{\kappa_{i} V_{i}^{n}}{\rho_{i}^{n}\left(c_{i}^{n}\right)^{2}}\left(\tilde{p}_{i}^{b u l k, n+1}-\bar{p}\right) \geq \sum_{k \in M(i)} \Psi_{i, k} \delta V_{i, k}^{\max } \geq 0, \quad \text { if } \tilde{p}_{i}^{\text {bulk,n+1}} \geq \bar{p} \\
& \frac{\kappa_{i} V_{i}^{n}}{\rho_{i}^{n}\left(c_{i}^{n}\right)^{2}}\left(\tilde{p}_{i}^{b u l k, n+1}-\bar{p}\right) \leq \sum_{k \in M(i)} \Psi_{i, k} \delta V_{i, k}^{\max } \leq 0, \quad \text { if } \tilde{p}_{i}^{b u l k, n+1} \leq \bar{p} .
\end{aligned}
$$

The model parameters are $\kappa_{b o t}$, which controls how close to zero the material volumes may be, and $\kappa_{i}$, which controls the rate of pressure relaxation. In the subsequent numerical section, the effect of these parameters upon the performance of the closure model is shown. Currently, the theory for the optimal choice of these parameters is not developed. The choice of the parameters is problem dependent, and the use of additional information about physics of the process may be utilized. For example, the rate of equilibration may be controlled based upon experimental data for a particular type of material. However, the important fact is that for the suggested range of values, the parameters guarantee minimal requirements which allows a problem to run to completion.

A summary of the input and output data for the IA-SSD closure model is now provided.

The input data for the closure model consists of the following quantities

- $\Delta V_{z}$ - change in the total volume of the multimaterial zone;

- $\alpha_{i}$-volume fraction for material $i \in M(z)$;

- $\delta V_{i, k}^{\max }$ - maximum allowable sub-scale volume exchange between materials $i$ and $k$;

- $p_{i, k}^{*}$ - the pressure which participates in the internal energy exchange term in the equation for material internal energy;

- parameters $\kappa_{b o t}$ and $\kappa_{i}$, which in this paper are taken to be global constants, are specified for each particular calculation in the numerical results section.

The constrained-optimization problem for finding the limiters $\Psi_{i, k}$ is evaluated as a quadratic optimization problem with linear inequality constraints, [31]. In the current implementation, the 'QL' software, [32], is used to solve this problem. 
4.3 Explicit solution of the constrained optimization problem in the case of two materials

It is educational to present an explicit solution of the constrained optimization problem in the case of a two-material zone. In this case, there is only one interface between materials \#1 and \#2, and only one unknown in the optimization problem: $\Psi_{1,2}$; for the sake of brevity, subscripts will be dropped and the limiter referred to as $\Psi$. For the same reason, the maximum volume exchange will be denoted by $\delta V^{\max }$ and the internal energy flux pressure $p^{*}$. The minimization problem, (55), in this case is

$$
\min _{\Psi}(1-\Psi)^{2}
$$

Note that the objective is a limiter in the range $0 \leq \Psi \leq 1$ which is monotonically decreasing on this interval. Therefore, the goal is to find the maximum value of $\Psi$ which satisfies the constraints.

The set of material volume related constraints, (56), is

$$
\begin{aligned}
& \Psi \delta V^{\max } \geq\left(\kappa_{\text {bot }}-1\right) V_{1}^{n+1, \text { bulk }}, \\
& -\Psi \delta V^{\max } \geq\left(\kappa_{\text {bot }}-1\right) V_{2}^{n+1, \text { bulk }} .
\end{aligned}
$$

Only one of these constraints can be active, for example, if $\delta V^{\max }>0$ then the first inequality is automatically satisfied because it is assumed that $\Psi \geq 0$ and the RHS is negative. Therefore only the second inequality is active and it gives following condition for $\Psi$

$$
\Psi \leq \Psi^{V}=\frac{\left(1-\kappa_{\text {bot }}\right) V_{2}^{n+1, \text { bulk }}}{\delta V^{\max }}, \quad \text { if } \delta V^{\max }>0 .
$$

In case when $\delta V^{\max }$ is negative, the first inequality is active

$$
\Psi \leq \Psi^{V}=\frac{\left(1-\kappa_{\text {bot }}\right) V_{1}^{n+1, \text { bulk }}}{\left|\delta V^{\max }\right|}, \quad \text { if } \delta V^{\max }<0 .
$$

If $\delta V^{\max }=0$ the limiter is set to unity, $\Psi^{V}=1$.

The set of the constraints related to the positivity of material internal energy, (57), is

$$
\begin{aligned}
& \mathcal{E}_{1}^{\text {bulk }} \geq \Psi p^{*} \Delta V^{\text {max }} \\
& \mathcal{E}_{2}^{\text {bulk }} \geq-\Psi p^{*} \Delta V^{\text {max }}
\end{aligned}
$$


As in the case of the volume related constraints only one of these constraints is active depending on sign of the energy flux $p^{*} \delta V^{\max }$ :

$$
\Psi \leq \Psi^{\varepsilon}=\frac{\mathcal{E}_{1}^{\text {bulk }}}{p^{*} \delta V^{\max }}, \quad \text { if } p^{*} \delta V^{\max }>0
$$

or

$$
\Psi \leq \Psi^{\varepsilon}=\frac{\mathcal{E}_{2}^{\text {bulk }}}{\left|p^{*} \delta V^{\max }\right|}, \quad \text { if } p^{*} \delta V^{\max }<0 .
$$

If $p^{*} \delta V^{\max }=0$ then the limiter is set to unity, $\Psi^{\varepsilon}=1$.

The constraints related to controlling pressure equilibration (58),(59) can be analyzed as follows.

There are two possible situations. In the first case $\tilde{p}_{1}^{b u l k, n+1} \geq \tilde{p}_{2}^{b u l k, n+1}$, so that

$$
\tilde{p}_{1}^{b u l k, n+1} \geq \bar{p} \geq \tilde{p}_{2}^{b u l k, n+1},
$$

and therefore the pressure equilibration constraints are

$$
\begin{aligned}
& \frac{\kappa_{1} V_{1}^{n}}{\rho_{1}^{n}\left(c_{1}^{n}\right)^{2}}\left(\tilde{p}_{1}^{b u l k, n+1}-\bar{p}\right) \geq \Psi \delta V^{\max } \geq 0, \\
& \frac{\kappa_{2} V_{2}^{n}}{\rho_{2}^{n}\left(c_{2}^{n}\right)^{2}}\left(\tilde{p}_{2}^{b u l k, n+1}-\bar{p}\right) \leq-\Psi \delta V^{\max } \leq 0 .
\end{aligned}
$$

If $\delta V^{\max }>0$ the solution for this system can be written as

$$
\Psi \leq \Psi^{p}=\min \left\{\frac{\kappa_{1} V_{1}^{n}}{\rho_{1}^{n}\left(c_{1}^{n}\right)^{2}} \frac{\left(\tilde{p}_{1}^{\text {bulk,n+1}}-\bar{p}\right)}{\delta V^{\max }}, \frac{\kappa_{2} V_{2}^{n}}{\rho_{2}^{n}\left(c_{2}^{n}\right)^{2}} \frac{\left(\bar{p}-\tilde{p}_{2}^{\text {bulk,n+1}}\right)}{\delta V^{\max }}\right\} \quad \text { if } \delta V^{\max }>0 .
$$

A graphical illustration of this expression is presented in Fig. 2. In this figure, the horizontal dotted lines represent the values of $\tilde{p}_{1}^{n+1, b u l k}, \tilde{p}_{2}^{n+1, b u l k}$ and $\bar{p}$. The top horizontal dashed line represents the value of $\kappa_{1} \bar{p}+\left(1-\kappa_{1}\right) \tilde{p}_{1}^{n+1, b u l k}$ and $\Psi$ needs to be chosen such that the value of $\tilde{p}_{1}^{n+1}(\Psi)$ lies between the upper horizontal dotted and dashed lines. A similar situation applies for $\tilde{p}_{2}^{n+1}(\Psi), \Psi$ must be chosen such that the value of $\tilde{p}_{2}^{n+1}(\Psi)$ lies between the lower dotted and dashed lines. In the situation presented in Fig. $2, \tilde{p}_{2}^{n+1}(\Psi)$ reaches its maximum allowable value faster than $\tilde{p}_{1}^{n+1}(\Psi)$ and so defines the value of $\Psi^{p}$. That is

$$
\tilde{p}_{2}^{n+1}\left(\Psi^{p}\right)=\kappa_{2} \bar{p}+\left(1-\kappa_{2}\right) \tilde{p}_{2}^{n+1, b u l k},
$$




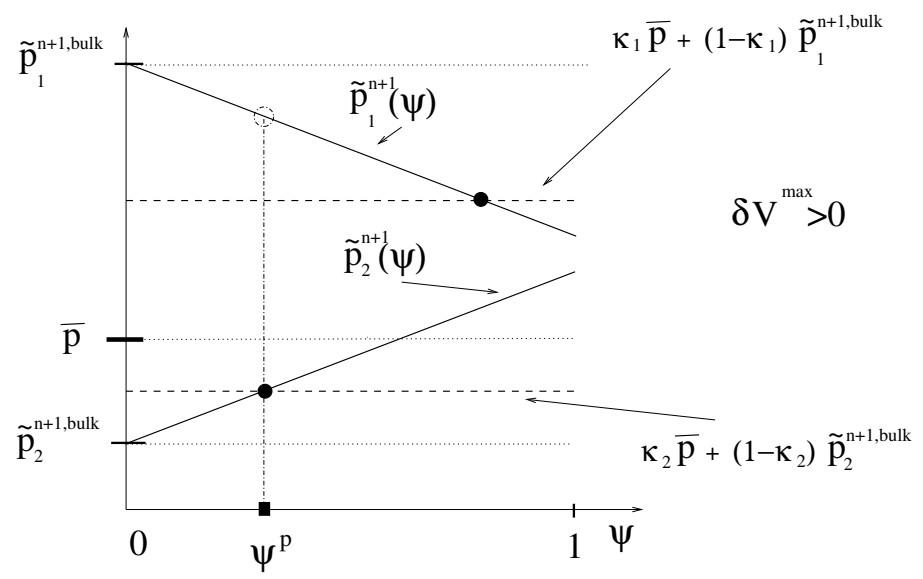

Fig. 2. Illustration of the determination of the limiter related to pressure equilibration, $\Psi^{p}$.

which gives the following expression for $\Psi^{p}$ :

$$
\Psi_{p}=\frac{\kappa_{2} V_{2}^{n}}{\rho_{2}^{n}\left(c_{2}^{n}\right)^{2}} \frac{\left(\bar{p}-\tilde{p}_{2}^{\text {bulk,n+1}}\right)}{\delta V^{\max }}
$$

The corresponding value of $\tilde{p}_{1}^{n+1}\left(\Psi^{p}\right)$ is marked by an empty circle in Fig. 2 .

If $\delta V^{\max }$ is negative, then the pressures $\tilde{p}_{1}^{n+1}(\Psi), \tilde{p}_{2}^{n+1}(\Psi)$ are diverging away from each other with increasing $\Psi$ and the only solution is

$$
\Psi^{p}=0 .
$$

Therefore for the case $\tilde{p}_{1}^{b u l k, n+1} \geq \bar{p} \geq \tilde{p}_{2}^{b u l k, n+1}$ the limiter related to pressure equilibration is defined by equations (72) and (75).

Now, consider the case when $\tilde{p}_{1}^{b u l k, n+1} \leq \tilde{p}_{2}^{\text {bulk,n+1}}$. Following similar logic, the pressure constraint is derived as

$$
\Psi \leq \Psi^{p}=\min \left\{\frac{\kappa_{1} V_{1}^{n}}{\rho_{1}^{n}\left(c_{1}^{n}\right)^{2}} \frac{\left(\bar{p}-\tilde{p}_{1}^{\text {bulk,n+1}}\right)}{\left|\delta V^{\max }\right|}, \frac{\kappa_{2} V_{2}^{n}}{\rho_{2}^{n}\left(c_{2}^{n}\right)^{2}} \frac{\left(\tilde{p}_{2}^{b u l k, n+1}-\bar{p}\right)}{\left|\delta V^{\max }\right|}\right\} \quad \text { if } \delta V^{\max }<0 .(76)
$$

and $\Psi^{p}=0$ if $\delta V^{\max }>0$. 


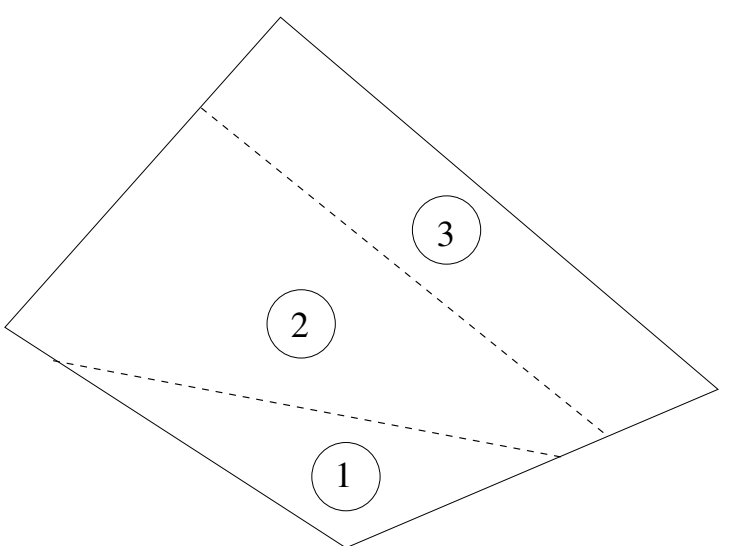

a)

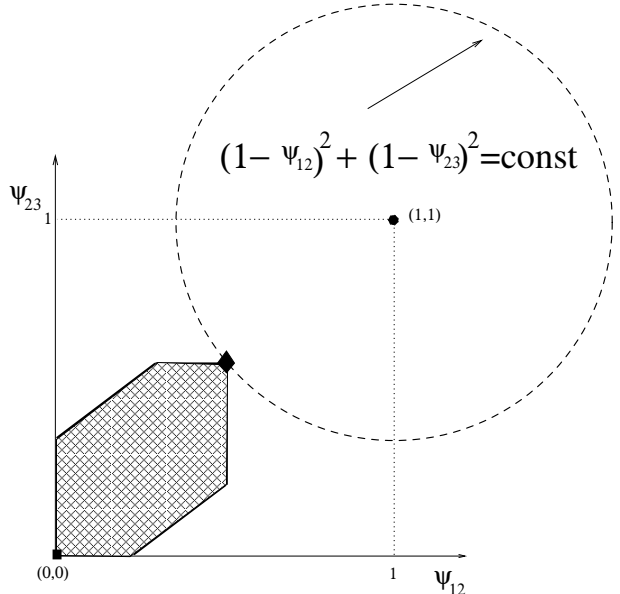

b)

Fig. 3. Illustration of the determination of values of limiters $\Psi_{1,2}, \Psi_{2,3}$ in the case of a layered configuration of three materials: a) the layered configuration; b) illustration of the constrained optimization problem.

The definition of $\Psi^{p}$ can be written in a more compact form

$$
\Psi^{p}=\left\{\begin{array}{l}
\left\{\begin{array}{l}
\text { if } p_{1}^{n+1, b u l k}>p_{2}^{n+1, b u l k} \text { and } \delta V^{\text {max }}<0 \\
\text { or } \\
\text { if } p_{1}^{n+1, b u l k}<p_{2}^{n+1, b u l k} \text { and } \delta V^{\text {max }}>0
\end{array}\right. \\
1 \quad \text { if } \delta V^{\text {max }}=0, \\
\min \left\{\frac{\kappa_{1} V_{1}^{n}}{\rho_{1}^{n}\left(c_{1}^{n}\right)^{2}} \frac{\left|\bar{p}-\tilde{p}_{1}^{\text {bulk,n+1}}\right|}{\left|\delta V^{\max }\right|}, \frac{\kappa_{2} V_{2}^{n}}{\rho_{2}^{n}\left(c_{2}^{n}\right)^{2}} \frac{\left|\tilde{p}_{2}^{\text {bulk,n+1}}-\bar{p}\right|}{\left|\delta V^{\max }\right|}\right\}, \text { otherwise }
\end{array}\right.
$$

The final value of the limiter $\Psi$ in the case of two materials is

$$
\Psi=\min \left\{\Psi^{V}, \Psi^{\varepsilon}, \Psi^{p}, 1\right\}
$$

\subsection{Constrained optimization problem: three materials, layered configuration - graphical illustration}

The simplest non-trivial example, for which a graphical illustration may be given, features three materials in a layered configuration, see Fig. 3 a). In this case there are only two interfaces, $1-2$ and $2-3$, therefore there are only two unknown limiters in the optimization problem: $\Psi_{1,2}$ and $\Psi_{2,3}$. The axis in Fig. 3 b) corresponds to these unknowns. The feasible set is a convex polygon, which is the cross-hatched region in this figure. Point $\Psi_{1,2}=\Psi_{2,3}=0$ belongs to this polygon. 
The aim of the constrained optimization problem is to find a point in the feasible set which is closest to $\Psi_{12}=\Psi_{23}=1$, with the objective function equal to zero. This point in the general case will lie on the boundary of the feasible set. This problem always has a unique solution.

\subsection{Time step control for a two-stage closure model}

The Courant stability condition (18) requires an expression for speed of sound for multimaterial cells.

In Section 3.3 the expression for speed of sound for the equal compressibility model - Equation (19) has been described. This expression is appropriate for the single-stage equal compressibility model. However, in the IA-SSD closure model, a second, sub-scale, stage is also applied where pressures are relaxed, and in the absence of external forces should equilibrate.

For models with equal pressures, and equal velocity in the case of two materials, [27] - Section 4.1, the speed of sound, $c_{z}$ for a mixture of two materials is defined by the equation

$$
\frac{1}{\rho_{z} c_{z}^{2}}=\frac{\alpha_{z, 1}}{\rho_{z, 1} c_{z, 1}^{2}}+\frac{\alpha_{z, 2}}{\rho_{z, 2} c_{z, 2}^{2}}
$$

where $\rho_{z}=\left(m_{1, z}+m_{2, z}\right) / V_{z}$ is the average density in the multimaterial zone. As mentioned in [27], this formula has an interesting and very important practical consequence: the speed of sound of the mixture is less than the sound speed of both components.

Analysis of Tipton's closure model (see, [28] and [4]) leads to the conclusion that equation (79) can be extended to an arbitrary number of materials

$$
\frac{1}{\rho_{z} c_{z}^{2}}=\sum_{i \in M(z)} \frac{\alpha_{z, i}}{\rho_{z, i} c_{z, i}^{2}}
$$

Therefore, two expressions for the speed of sound in a multimaterial cell are available. The first is (19), which is appropriate for the equal compressibility model, in which pressures are different and do not equilibrate. The second is (80), which is appropriate for the situation when the pressures of the materials are equal. It is natural to assume that depending on the level of pressure equilibration, the speed of sound in the IA-SSD model can be estimated by some interpolation between the expressions given by (19) and (80). The following 
heuristic formula is suggested,

$$
c_{z}=\beta_{z} c_{z}^{e v s}+\left(1-\beta_{z}\right) c_{z}^{\text {peq }}, \quad 1 \geq \beta_{z} \geq 0
$$

where $\beta_{z}$ should be equal to zero when all pressures are the same, and $\beta_{z}$ should approach unity when the material pressures are very different; in equation (81) $c_{z}^{\text {evs }}$ corresponds to expression (19) and $c_{z}^{p e q}$ corresponds to expression (80).

For two materials, the following ad-hoc expression for $\beta_{z}$ is used

$$
\beta_{z}=\frac{1}{2} \cdot \frac{\left(p_{z, 1}-p_{z, 2}\right)^{2}}{p_{z, 1}^{2}+p_{z, 2}^{2}}
$$

Defining $\beta_{z}$ in this way results in zero if the two pressures are the same, and one if one pressure is $+\infty$ and another pressure is $-\infty$. Therefore this form satisfies the limiting cases and allows a smooth transition between them. It can also be noted that for the two-material case, an expression for the speed of sound which takes into account the level of pressure equilibration similar to (80) has been derived in [36] (Eq. 50). The expression in [36] is similar to (80), however the formula for $\beta_{z}$ is different, and it also features a parameter which controls the level of relaxation. Future work may compare calculations for different choices of $\beta_{z}$, including the form derived in [36].

In the case of $\mathcal{M}=|M(z)|>2$ materials $^{8}$ in the multimaterial zone $z$, the previous expression for $\beta_{z}$ can be extended as follows

$$
\beta_{z}=\frac{\mathcal{M}}{4 N} \frac{\sum_{(i, k)}\left(p_{z, i}-p_{z, k}\right)^{2}}{\sum_{i \in M(z)} p_{z, i}^{2}}
$$

where $(i, k)$ belongs to the unordered subsets containing 2 elements from $M(z)$, the number of such elements is $N=\frac{\mathcal{M}(\mathcal{M}-1)}{2}$.

An alternate approach to determine the average sound speed for a multimaterial cell is to estimate the transit time for a wave to cross such a zone. Two good approximations, which bound the solution, can be obtained by considering the materials in the zone to be arranged in layers. In this case, the materials may be in either parallel or orthogonal orientations. The lower limit for the transit time will correspond to the parallel case, with the wave traveling all the way across the zone through the fastest shock speed material. The transit time will therefore be determined by the highest sound speed of the material components. The upper limit will be the orthogonal case, and will be best represented by a mass fraction average, where the total transit time will

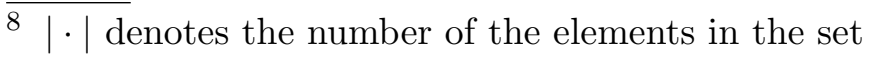




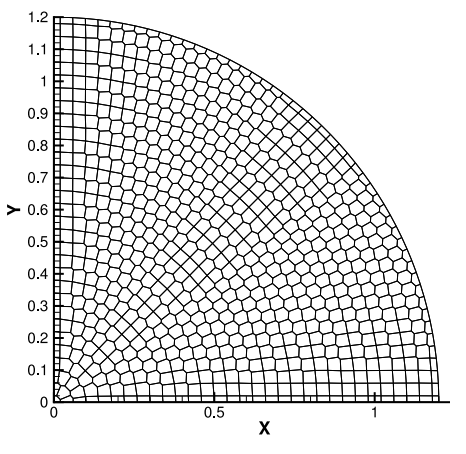

a)

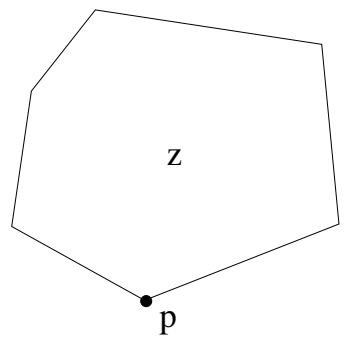

b)

Fig. 4. General polygonal mesh: a) Entire grid, b) Zone and corresponding notations.

be the sum of the times to cross the individual layers in turn. The average sound speed should only be outside these bounds if the materials are mixed. The sensitivity of the closure model to the type of average used for the sound speed of the multimaterial cell is a topic for future research.

\section{Compatible staggered discretization for multimaterial Lagrangian hydrodynamics}

A description is now given detailing how the new interface aware subscale closure model is incorporated into the framework of the compatible total energy conserving discretization of multimaterial Lagrangian hydrodynamics with multimaterial cells.

5.1 Compatible staggered discretization for Lagrangian hydrodynamics - single material

First, the single material case is considered in order to aid the explanation of the multimaterial cell case.

\subsubsection{Semi-discrete equations}

For the simplicity of presentation, the semi-discrete case is first considered; that is, time remains continuous, and only the spatial discretization is considered. In this paper, a general polygonal mesh is considered, see Fig. 4. 
Note that in the staggered discretization, point quantities include the spatial coordinates, $\left(x_{p}, y_{p}\right)$, velocity components, $\left(u_{p}, v_{p}\right)$, and point mass $m_{p}$. Zonal quantities are volume, $V_{z}$, density, $\rho_{z}$, mass, $m_{z}$, internal energy, $\varepsilon_{z}$, and pressure $p_{z}$.

For Lagrangian methods $d\left(m_{z}\right) / d t=0$ with $m_{z}=\left.\left.\rho_{z}\right|_{t=0} \cdot V_{z}\right|_{t=0}$. Therefore, for any time moment, $t$, density can be defined as follows $\rho_{z}(t)=m_{z} / V_{z}(t)$, which plays the role of a discrete continuity equation. Also, for Lagrangian methods each point moves with the fluid velocity, that is, $d x_{p} / d t=u_{p}, d y_{p} / d t=v_{p}$.

The generic compatible form of the discrete momentum and internal energy equations for case of single material is (see [26])

$$
\begin{aligned}
& m_{p} \frac{d \mathbf{u}_{p}}{d t}=\sum_{z \in \mathcal{Z}(p)} \mathbf{f}_{z p} \\
& m_{z} \frac{d \varepsilon_{z}}{d t}=-\sum_{p \in \mathcal{P}(z)} \mathbf{f}_{z p} \cdot \mathbf{u}_{p}
\end{aligned}
$$

where $\mathbf{f}_{z p}$ denotes so-called sub-zonal forces; $\mathcal{Z}(p)$ is set of zones which share node $p$, and $\mathcal{P}(z)$ is set of vertices of zone $z$.

Therefore the spatial discretization is completely defined with the definition of the sub-zonal forces $\mathbf{f}_{z p}$. In the simplest case, which is considered in this paper, sub-zonal forces depend on the geometry of the zone and the zonal pressure.

Total kinetic energy is defined as

$$
K=\sum_{p} K_{p}, \quad K_{p}=m_{p} \frac{\left|\mathbf{u}_{p}\right|^{2}}{2}
$$

where the summation is performed over all points (vertices) of the mesh.

The total internal energy is defined as

$$
\mathcal{E}=\sum_{z} \mathcal{E}_{z}, \quad \mathcal{E}_{z}=m_{z} \varepsilon_{z}
$$

where the summation is performed over all zones (cells) of the mesh.

Now, the definition of the sub-zonal force $\mathbf{f}_{z p}$ is required. The discrete equation (84) must approximate the momentum equation, (2), which implies that the 


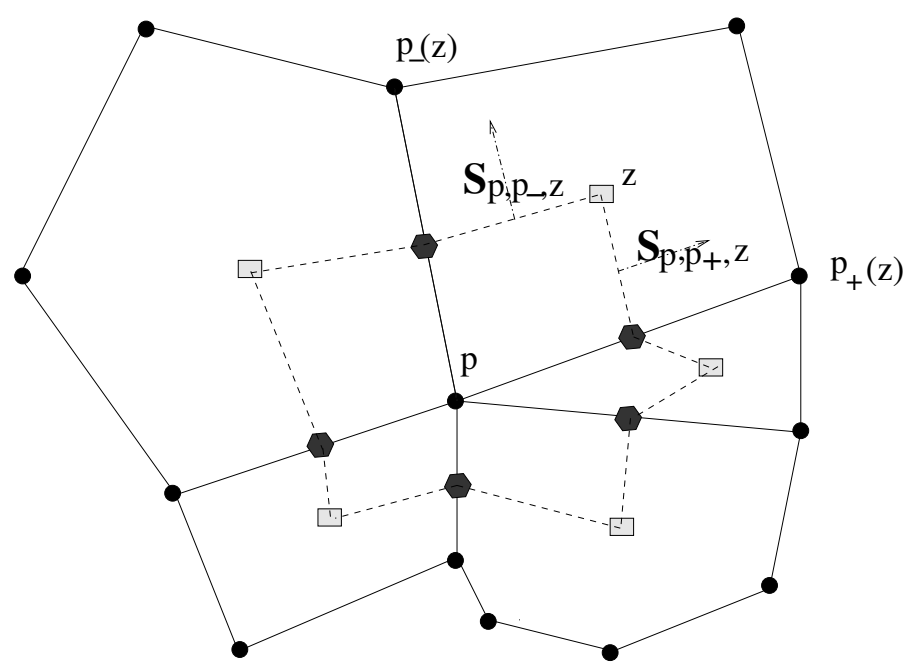

Fig. 5. Dual volume $V_{p}$ and definition of $S_{z p}$.

sub-zonal forces are constructed such that

$$
\sum_{z \in \mathcal{Z}(p)} \mathbf{f}_{z p} \approx \oint_{\partial V_{p}} p \mathbf{d} \mathbf{S}
$$

where $V_{p}$ is the volume surrounding the point $p$ which is constructed by connecting the midpoints of the sides having point $p$ as one vertex to the geometric centers of the zones which share point $p$ - see Fig. 5 .

The explicit expression for $\mathbf{f}_{z p}$ is

$$
\mathbf{f}_{z p}=\mathbf{S}_{z p} p_{z}, \quad \mathbf{S}_{z p}=\mathbf{S}_{p, p^{+}, z}+\mathbf{S}_{p, p^{-}, z}
$$

where $\mathbf{S}_{p, p^{+}, z}, \mathbf{S}_{p, p^{-}, z}$ are vectors which are directed in the direction of the outward normals to the sides of $V_{p}$ with magnitude equal to the length of the corresponding sides - Fig. 5.

Taking into account equation (89) for $\mathbf{f}_{z p}$, the discrete equation (85) can be rewritten for internal energy as

$$
m_{z} \frac{d \varepsilon_{z}}{d t}=-p_{z} \sum_{p \in \mathcal{P}(z)} \mathbf{S}_{z p} \cdot \mathbf{u}_{p}
$$

As it is shown in $[26,46]^{9}$

$$
\sum_{p \in \mathcal{P}(z)} \mathbf{S}_{z p} \cdot \mathbf{u}_{p}=\frac{d V_{z}}{d t} .
$$

9 Note that discretization in time has not yet been performed. 
Therefore, the discrete equation (85) is indeed an approximation of the continuum internal energy equation in the form (6).

\subsection{Fully discrete equation - single material}

In this Section, the complete generic predictor-corrector time integration method for the compatible spatial discretization for a single material is described. This description is needed in the future presentation when the time integration with the presence of multimaterial cells is explained.

\section{Predictor stage}

The momentum equation is discretized as follows:

$$
m_{p} \frac{\mathbf{u}_{p}^{n+1, p r}-\mathbf{u}_{p}^{n}}{\Delta t}=\sum_{z \in \mathcal{Z}(p)}\left(\mathbf{f}^{p}\right)_{z p}^{n}+\sum_{z \in \mathcal{Z}(p)}\left(\mathbf{f}^{a}\right)_{z p}^{n}
$$

where the first term on the RHS corresponds to pressure forces and the second term contains all artificial forces such as artificial viscosity and anti-hourglass. The superscript $n$ in the notation for sub-zonal forces indicates that all expressions (including geometric quantities) used in its definition are computed at $t^{n}$

The new predicted velocity, $\mathbf{u}_{p}^{n+1, p r}$, allows the computation of new spatial coordinates, volumes and densities

$$
\begin{aligned}
& \mathbf{x}_{p}^{n+1, p r}=\mathbf{x}_{p}^{n}+\mathbf{u}_{p}^{n+1 / 2, p r} \Delta t, \quad \mathbf{u}_{p}^{n+1 / 2, p r}=\frac{1}{2}\left(\mathbf{u}_{p}^{n+1, p r}+\mathbf{u}_{p}^{n}\right) \\
& V_{z}^{n+1, p r}=\mathcal{V}\left(\mathbf{x}_{p}^{n+1, p r} ; p \in \mathcal{P}(z)\right) \\
& \rho_{z}^{n+1, p r}=m_{z} / V_{z}^{n+1, p r}
\end{aligned}
$$

Next the internal energy equation is approximated as

$$
m_{z} \frac{\varepsilon_{z}^{n+1, p r}-\varepsilon_{z}^{n}}{\Delta t}=-\sum_{p \in \mathcal{P}(z)}\left(\left(\mathbf{f}^{p}\right)_{z p}^{n} \cdot \mathbf{u}_{p}^{n+1 / 2, p r}\right)+Q_{z}^{a, n}
$$

where $Q_{z}^{a, n}$ is the total heat from artificial forces

$$
Q_{z}^{a, n}=-\sum_{p \in \mathcal{P}(z)}\left(\left(\mathbf{f}^{a}\right)_{z p}^{n} \cdot \mathbf{u}_{p}^{n+1 / 2, p r}\right)
$$


Finally, the material pressures are updated as

$$
p_{z}^{n+1, p r}=\operatorname{EOS}\left(\rho_{z}^{n+1, p r}, \varepsilon_{z}^{n+1, p r}\right) .
$$

\section{Corrector stage}

The corrector stage is similar to the predictor stage. The main difference is that for the computation of sub-zonal forces, information obtained at the predictor stage is used. Introducing the generic notation

$$
\theta_{b}^{n+1 / 2}=0.5\left(\theta_{b}^{n}+\theta_{b}^{n+1}\right)
$$

where $b$ is some generic index, the formulas comprising the corrector stage can be written as follows:

$$
\begin{aligned}
& m_{p} \frac{\mathbf{u}_{p}^{n+1}-\mathbf{u}_{p}^{n}}{\Delta t}=\sum_{z \in \mathcal{Z}(p)}\left(\mathbf{f}^{p}\right)_{z p}^{n+1 / 2}+\sum_{z \in \mathcal{Z}(p)}\left(\mathbf{f}^{a}\right)_{z p}^{n+1 / 2}, \\
& \mathbf{x}_{p}^{n+1}=\mathbf{x}_{p}^{n}+\mathbf{u}_{p}^{n+1 / 2} \Delta t, \quad \mathbf{u}_{p}^{n+1 / 2}=\frac{1}{2}\left(\mathbf{u}_{p}^{n+1}+\mathbf{u}_{p}^{n}\right), \\
& V_{z}^{n+1}=\mathcal{V}\left(\mathbf{x}_{p}^{n+1} ; p \in \mathcal{P}(z)\right), \\
& \rho_{z}^{n+1}=m_{z} / V_{z}^{n+1}, \\
& m_{z} \frac{\varepsilon_{z}^{n+1}-\varepsilon_{z}^{n}}{\Delta t}=-\sum_{p \in \mathcal{P}(z)}\left(\left(\mathbf{f}^{p}\right)_{z p}^{n+1 / 2} \cdot \mathbf{u}_{p}^{n+1 / 2}\right)+Q_{z}^{a, n+1 / 2}, \\
& p_{z}^{n+1}=\operatorname{EOS}\left(\rho_{z}^{n+1}, \varepsilon_{z}^{n+1}\right),
\end{aligned}
$$

with

$$
Q_{z}^{a, n+1 / 2}=-\sum_{p \in \mathcal{P}(z)}\left(\left(\mathbf{f}^{a}\right)_{z p}^{n+1 / 2} \cdot \mathbf{u}_{p}^{n+1 / 2}\right) .
$$

5.3 Compatible equations for momentum and material internal energies in presence of the multimaterial zones

For multimaterial zones, the averaged pressure is defined by equation (16), therefore the sub-zonal forces in the compatible formulation of the momentum equation must then be defined as

$$
\left(\mathbf{f}_{z p}^{\tilde{p}}\right)^{n}=\left\{\sum_{i \in M(z)}\left[\alpha_{z, i}^{n} p_{z, i}^{n}\right]\right\} \mathbf{S}_{z p}^{n} .
$$


This expression may be rewritten as

$$
\left(\mathbf{f}^{\tilde{p}}\right)_{z p}^{n}=\tilde{p}_{z}^{n} \mathbf{S}_{z p}^{n}=\sum_{i \in M(z)}\left(\alpha_{z, i}^{n} p_{z, i}^{n}\right) \mathbf{S}_{z p}^{n}=\sum_{i \in M(z)}\left(\alpha_{z, i}^{n} p_{z, i}^{n} \mathbf{S}_{z p}^{n}\right)=\sum_{i \in M(z)}\left(\mathbf{f}^{p_{i}}\right)_{z p}^{n}(108)
$$

where the sub-zonal material force $\left(\mathbf{f}^{p_{i}}\right)_{z p}^{n}$ is introduced as

$$
\left(\mathbf{f}^{p_{i}}\right)_{z p}^{n}=\alpha_{z, i}^{n} p_{z, i}^{n} \mathbf{S}_{z p}^{n} .
$$

Therefore, the total pressure force in the momentum equation can be written as

$$
\sum_{z \in \mathcal{Z}(p)}\left(\mathbf{f}^{\tilde{p}}\right)_{z p}^{n}=\sum_{z \in \mathcal{Z}(p)}\left[\sum_{i \in M(z)}\left(\mathbf{f}^{p_{i}}\right)_{z p}^{n}\right]=\sum_{i \in M(z)}\left[\sum_{z \in \mathcal{Z}(p)}\left(\mathbf{f}^{p_{i}}\right)_{z p}^{n}\right]
$$

and so the momentum equation, for example, for the predictor stage, can be written as

$$
m_{p} \frac{\mathbf{u}_{p}^{n+1, p r}-\mathbf{u}_{p}^{n}}{\Delta t}=\sum_{i \in M(z)}\left[\sum_{z \in \mathcal{Z}(p)}\left(\mathbf{f}^{p_{i}}\right)_{z p}^{n}\right] .
$$

The compatible internal energy equation for material $i$, which is consistent with (42) is

$$
m_{z, i}\left(\varepsilon_{z, i}^{n+1, p r}-\varepsilon_{z, i}^{n}\right)=-\Delta t \sum_{p \in \mathcal{P}(z)}\left(\left(\mathbf{f}^{p_{i}}\right)_{z p}^{n} \cdot \mathbf{u}_{p}^{n+1 / 2, p r}\right)+\sum_{k \in M_{z}(i)} \Psi_{i, k} p_{i, k}^{*} \delta V_{i, k}^{\max , n}+Q_{z, i}^{n},(11
$$

The details of derivation of compatible total energy conserving equations for momentum equation and material energies can be found in [28].

\section{Summary of algorithm}

In this section a description is given of one possible algorithm which uses the simplest choice of input data in the optimization procedure.

\section{Predictor stage}

The predictor stage starts with the computation of the average pressure

$$
\tilde{p}_{z}^{n}=\sum_{i \in M(z)} \alpha_{z, i}^{n} p_{z, i}^{n} .
$$


This is done exactly as for the equal compressibility model.

The momentum equation is discretized in a similar manner to the case of the equal compressibility model, however, the artificial viscosity forces may be computed differently,

$$
m_{p} \frac{\mathbf{u}_{p}^{n+1, p r}-\mathbf{u}_{p}^{n}}{\Delta t}=\sum_{z \in \mathcal{Z}(p)}\left(\mathbf{f}^{\tilde{p}}\right)_{z p}^{n}+\sum_{z \in \mathcal{Z}(p)}\left(\mathbf{f}^{a}\right)_{z p}^{n} .
$$

The new predicted velocity, $\mathbf{u}_{p}^{n+1, p r}$, allows the computation of new spatial coordinates and the volumes of all zones

$$
\begin{aligned}
& \mathbf{x}_{p}^{n+1, p r}=\mathbf{x}_{p}^{n}+\mathbf{u}_{p}^{n+1 / 2, p r} \Delta t, \quad \mathbf{u}_{p}^{n+1 / 2, p r}=\frac{1}{2}\left(\mathbf{u}_{p}^{n+1, p r}+\mathbf{u}_{p}^{n}\right) \\
& V_{z}^{n+1, p r}=\mathcal{V}\left(\mathbf{x}_{p}^{n+1, p r} ; p \in \mathcal{P}(z)\right)
\end{aligned}
$$

Now, the total change in zonal volume may be defined as

$$
\Delta V_{z}^{n+1, p r}=V_{z}^{n+1, p r}-V_{z}^{n} .
$$

Next, $\delta V_{i, k}^{\max }$ may be calculated, in this case, using data from the previous time step

$$
\delta V_{i, k}^{\max , n}=\frac{p_{i}^{n}-p_{k}^{n}}{\rho_{i}^{n} c_{i}^{n}+\rho_{k}^{n} c_{k}^{n}} S_{i, k}^{n} \Delta t
$$

In particular, this means that $S_{i, k}^{n}$ is computed using the interface reconstruction data from $t^{n}$.

The pressure $p_{i, k}^{*}$ is also computed using data from the previous time step

$$
p_{i, k}^{*, n}=\mathcal{P}^{*}\left(\rho^{n}, c^{n}, p^{n}, \mathbf{u}^{n}\right)=\frac{\left(\rho_{k}^{n} c_{k}^{n}\right) p_{i}^{n}+\left(\rho_{i}^{n} c_{i}^{n}\right) p_{k}^{n}-\left(\rho_{k}^{n} c_{k}^{n}\right)\left(\rho_{i}^{n} c_{i}^{n}\right)\left(\mathbf{u}_{k}^{n}-\mathbf{u}_{i}^{n}\right) \cdot \mathbf{n}_{i, k}^{n}}{\rho_{k}^{n} c_{k}^{n}+\rho_{i}^{n} c_{i}^{n}}
$$

Parameters $\kappa_{\text {bot }}$ and $\left\{\kappa_{i}\right\}$ may then be specified.

At this stage, the closure model may be applied,

$$
\Psi_{i, k} \leftarrow \text { CON_OPT }\left(\Delta V_{z}^{n+1, p r}, \delta V_{i, k}^{\max , n}, p_{i, k}^{*, n}, \kappa_{b o t}, \kappa_{i}\right),
$$

where CON_OPT indicates the application of the constrained optimization procedure. 
The new volumes for each material are then defined as

$$
V_{z, i}^{n+1, p r}=\alpha_{z, i}^{n} V_{z}^{n+1, p r}+\sum_{k \in M_{z}(i)} \Psi_{i, k} \delta V_{i, k}^{\max , n}
$$

Using the updated material volumes, the material densities are updated,

$$
\rho_{z, i}^{n+1, p r}=m_{z, i} / V_{z, i}^{n+1, p r} .
$$

Next, an approximation of the internal energy equation

$$
m_{z, i} \frac{\varepsilon_{z, i}^{n+1, p r}-\varepsilon_{z, i}^{n}}{\Delta t}=-\sum_{p \in \mathcal{P}(z)}\left(\left(\mathbf{f}^{p_{i}}\right)_{z p}^{n} \cdot \mathbf{u}_{p}^{n+1 / 2, p r}\right)+\sum_{k \in M_{z}(i)} \Psi_{i, k} p_{i, k}^{*, n} \frac{\delta V_{i, k}^{\max , n}}{\Delta t}+Q_{z, i}^{n}
$$

and finally the material pressures are updated,

$$
p_{z, i}^{n+1, p r}=\operatorname{EOS}\left(\rho_{z, i}^{n+1, p r}, \varepsilon_{z, i}^{n+1, p r}\right) .
$$

\section{Corrector stage}

$$
\begin{aligned}
& \tilde{p}_{z}^{n+1}=\sum_{i \in M(z)} \alpha_{z, i}^{n} p_{z, i}^{n+1, p r}, \\
& m_{p} \frac{\mathbf{u}_{p}^{n+1}-\mathbf{u}_{p}^{n}}{\Delta t}=\sum_{z \in \mathcal{Z}(p)}\left(\mathbf{f}^{\tilde{p}}\right)_{z p}^{n+1 / 2}+\sum_{z \in \mathcal{Z}(p)}\left(\mathbf{f}^{a}\right)_{z p}^{n} \\
& \mathbf{x}_{p}^{n+1}=\mathbf{x}_{p}^{n}+\mathbf{u}_{p}^{n+1 / 2} \Delta t, \quad \mathbf{u}_{p}^{n+1 / 2}=\frac{1}{2}\left(\mathbf{u}_{p}^{n+1}+\mathbf{u}_{p}^{n}\right) \\
& V_{z}^{n+1}=\mathcal{V}\left(\mathbf{x}_{p}^{n+1} ; p \in \mathcal{P}(z)\right) . \\
& \Delta V_{z}^{n+1}=V_{z}^{n+1}-V_{z}^{n}, \\
& \delta V_{i, k}^{\text {max }}, n+1=\frac{p_{i}^{n+1 / 2, p r}-p_{k}^{n+1 / 2, p r}}{\rho_{i}^{n+1 / 2, p r}} c_{i}^{n+1 / 2, p r}+\rho_{k}^{n+1 / 2, p r} c_{k}^{n+1 / 2, p r} S_{i, k}^{n+1} \Delta t . \\
& p_{i, k}^{*, n+1}=\mathcal{P}^{*}\left(\rho^{n+1 / 2, p r}, c^{n+1 / 2, p r}, p^{n+1 / 2, p r}, \mathbf{u}^{n+1 / 2, p r}\right) \\
& \Psi_{i, k} \leftarrow \operatorname{CON} \mathrm{OPT}\left(\Delta V_{z}^{n+1}, \delta V_{i, k}^{\max , n+1}, p_{i, k}^{*, n+1}, \kappa_{b o t}, \kappa_{i}\right) \\
& V_{z, i}^{n+1}=\alpha_{z, i}^{n} V_{z}^{n+1}+\sum_{k \in M_{z}(i)} \Psi_{i, k} \delta V_{i, k}^{\max , n+1},
\end{aligned}
$$




$$
\begin{aligned}
& \rho_{z, i}^{n+1}=m_{z, i} / V_{z, i}^{n+1} \\
& m_{z, i} \frac{\varepsilon_{z, i}^{n+1}-\varepsilon_{z, i}^{n}}{\Delta t}=-\sum_{p \in \mathcal{P}(z)}\left(\left(\mathbf{f}^{p_{i}}\right)_{z p}^{n+1 / 2} \cdot \mathbf{u}_{p}^{n+1 / 2}\right)+\sum_{k \in M_{z}(i)} \Psi_{i, k} p_{i, k}^{*, n+1} \delta V_{i, k}^{\text {max }, n+1}+Q_{z, i}^{n+1 / 2},(13) \\
& p_{z, i}^{n+1}=\operatorname{EOS}\left(\rho_{z, i}^{n+1}, \varepsilon_{z, i}^{n+1}\right)
\end{aligned}
$$

\section{Time evolution of the centroids of the materials in Lagrangian gas dynamics and closure models}

IA-SSD closure models require knowledge of the interfaces between materials in the multimaterial cell, Fig.1. This information comes from the interface reconstruction algorithm. One of the most promising interface reconstruction algorithms is the moment-of-fluid (MOF) method [24,23]. This method requires knowledge of the volume fraction of each material along with a reference centroid of the material. The MOF method is optimization-based and it finds the material configuration such that the areas of pure sub-polygons representing materials match specified volume fractions exactly and the discrepancy between the reference and actual centroids is minimized.

Therefore in the framework of Lagrangian methods which use IA-SSD closure models, and for multimaterial ALE methods, a procedure for advancing centroids in time during Lagrangian step is required. In previous publications [47], [23], the positions of the centroids at a new time moment, $t^{n+1}$, were obtained using a velocity interpolated to the centroid from the nodal velocities of the multimaterial cell and transported as a passive scalar from the position at $t^{n}$. These positions are presented in Fig.1 as solid circles and approximately correspond to the centroids of the deformed material polygons at time $t^{n+1}$. It is obvious that these positions do not take into account updates from sub-scale interactions, and only capture the bulk movement of the entire multimaterial cell. If these centroids are used as reference centroids for the MOF interface reconstruction, some inconsistency with the closure model may arise, see [25].

A new method to update material centroid positions is suggested which is consistent with the IA-SSD closure model. The preliminary centroid positions corresponding to the bulk movement are denoted by $\mathbf{x}_{c, i}^{b u l k, n+1}$. It is assumed that

the bulk movement gives the volumes of the materials, $V_{i}^{b u l k, n+1}$, as described by the IA-SSD closure model. Centroids of the rectangles corresponding to the relative volume exchanges, $\delta V_{i, k}^{n}$, are denoted by $\mathbf{x}_{c ;(i, k)}^{n}$. These centroids are marked by solid rectangles in Fig.1. 
Using this notation, the final reference centroid for material $i$ is defined as

$$
\mathbf{x}_{c, i}^{n+1}=\frac{V_{i}^{b u l k, n+1} \mathbf{x}_{c, i}^{b u l k, n+1}+\sum_{k \in M(i)} \delta V_{i, k}^{n} \mathbf{x}_{c ;(i, k)}^{n}}{V_{i}^{n+1}} .
$$

In the examples presented in Section 8, the recently developed symmetric version of the MOF method, [25], is used which improves the geometric fidelity of the original MOF method.

\section{Numerical examples}

First, in order to evaluate the closure models, a series of simple and easy to reproduce one-dimensional Lagrangian test cases are performed which isolate and replicate key situations that may occur in multimaterial cells. Lagrangian simulations are initially performed due to their increased sensitivity to the choice of closure model.

The first class of test cases simulates the case where the initial mesh is not aligned with the material interface so that a material contact exists in a multimaterial cell from the beginning of the simulation. At some later time, some wave will then interact with the contact. The performance of the available closure models in such cases is examined, with reference also made to equivalent pure cell calculations.

A second class of test cases replicates situations which arise following an ALE rezone stage. The rezoning stage can introduce a multimaterial cell containing two materials with very different properties. This situation corresponds to the general Riemann problem. The rezone stage may also introduce multimaterial cells which feature a small volume fraction for one of the materials. The treatment of sub-scale interactions between materials will be of particular influence in these cases.

Next, the modified Sod problem, and the water-air problem are considered featuring a two-material cell in which the materials have different equations of state. These tests are more representative of multimaterial simulations, and allow a wider examination of the closure models.

One-dimensional examples are concluded with an analysis of pressure equilibration in a three-material cell.

Two-dimensional Lagrangian test cases are then examined. These simulations allow analysis of multidimensional aspects, such as misaligned contacts with 
respect to the computational mesh, and preservation of symmetry with multiple (more than two) materials, to be evaluated with respect to the choice of closure model.

Finally, classical ALE simulations are performed which feature many of the situations previously considered in isolation to provide a view of the overall performance of the new IA-SSD closure model with respect to benchmark results.

\subsection{D examples - Lagrangian hydrodynamics}

In all calculations, an initially uniform mesh is used. For most problems, pure cell calculations are presented as a reference solution. The basic Lagrangian scheme uses a combination of linear and quadratic artificial viscosity as described in [26], the coefficients of linear and quadratic viscosities are $q_{1}=1$, $q_{2}=0.1$ respectively. The default values of the parameters in IA-SSD constraints are $\kappa_{i}=0.99$ and $\kappa_{\text {bot }}=0.5$.

\subsubsection{Sanity checks - single material calculations}

Here, results are presented for several tests where a 'fake' two material cell is created - both of the materials have the same equation of state, but may have initially different state variables. These types of tests are termed "sanity checks" in the sense that the solution should not be disturbed significantly by the introduction of a multimaterial cell. At first glance this may look somewhat artificial, however, in real multidimensional calculations, it is not always possible to create a computational mesh which is aligned with discontinuities even in a single material. In this case multimaterial cells may be used to more accurately represent initial conditions.

In order to evaluate the performance of the closure models, a reference solution is obtained for particular time and spatial discretizations (including artificial viscosity) via Lagrangian calculations with pure cells. The computational mesh is uniform such that discontinuity coincides with a vertex of the cell, that is, all cells are pure and contain only one material. In order to obtain a comparable multimaterial simulation, the underlying mesh remains the same, but the position of the initial discontinuity is shifted by some percentage of the mesh size so that it lies within the first cell to the right of the discontinuity.

Interaction of the shock wave with fake contact

In [28] a series of test cases have been presented where different types of wave interact with an artificial material interface between identical materials. 


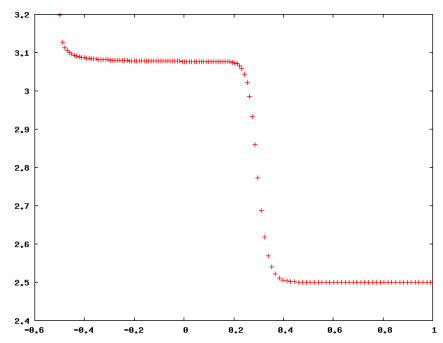

a)

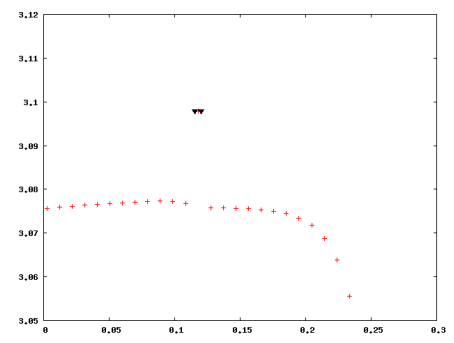

b)

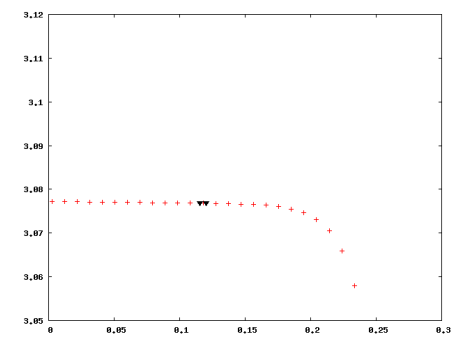

c)

Fig. 6. Shock wave, 128 cells: a)Pure cell calculation - internal energy; b), c) Interaction of the shock wave with a fake contact between identical materials in the multimaterial cell - internal energy: b) Tipton's model - magnified view, c) IA-SSD model - magnified view.

Such waves include a stationary shock wave, a Sedov-like blast wave and a rarefaction wave. All these examples exhibit similar features and for this reason only results for the interaction of the shock with a fake contact are presented in this paper.

The initial computational domain is $[-1: 1]$. The left boundary is a piston with velocity $u=0.620174$ which generates a shock. The right boundary is a wall. The initial conditions are $\rho=1, p=1$ and the material is an ideal gas with $\gamma=1.4$; the final time is $t=0.8$. The simulations use 128 cells. Multimaterial simulations feature a two-material cell immediately to the right of the origin (cell number 65) $-\alpha_{l}=\alpha_{r}=0.5$.

Figure 6 compares profiles of internal energy for the pure cell, Tipton and IASSD simulations. For multimaterial calculations, magnified views around the multimaterial cell are presented, mass averaged internal energy is presented, $\varepsilon=\left(m_{1} / m\right) \varepsilon_{1}+\left(m_{2} / m\right) \varepsilon_{2}$, where $m_{i}=V_{i} \rho_{i}, i=1,2$ is the mass of material $i$ in the multimaterial cell, and $m=m_{1}+m_{2}$ is the total mass. The material internal energies are also shown in figures by black solid triangles. It can be seen that Tipton's model features an overshoot in the material internal energies and the cell-averaged internal energy. The IA-SSD model does not introduce any artifacts in the numerical solution.

\section{Artificial interface (single material) Sod problem as an example Rie- mann problems "inside" a multimaterial cell}

Similarly to the previous section, a series of example Riemann problems "inside" the multimaterial cell: isolated shock, isolated rarefaction, isolated contact, Sod problem have been presented in [28]. All these examples have similar features, and for this reason are omitted from this paper, with a concentration on the Sod problem provided instead. The computational domain is a segment with bounds $\left[x_{0}: x_{1}\right]=[0: 1]$. The material is an ideal gas with $p=(\gamma-1) \rho \varepsilon, \gamma=1.4$. The boundary conditions are $u(0)=u(1)=0$. The 


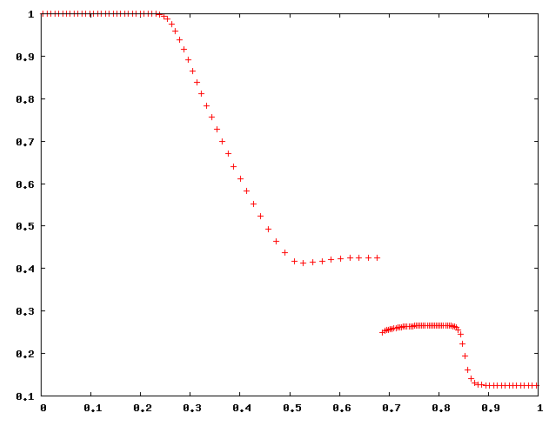

a)

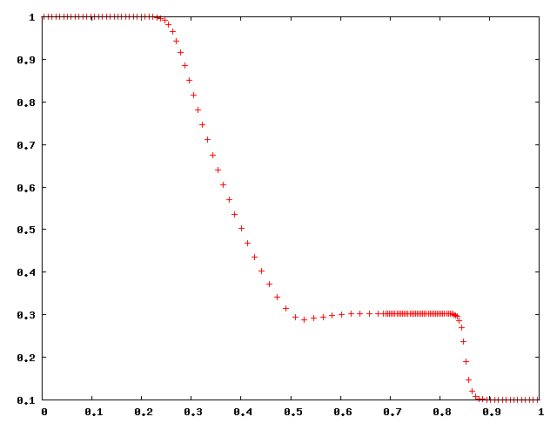

c)

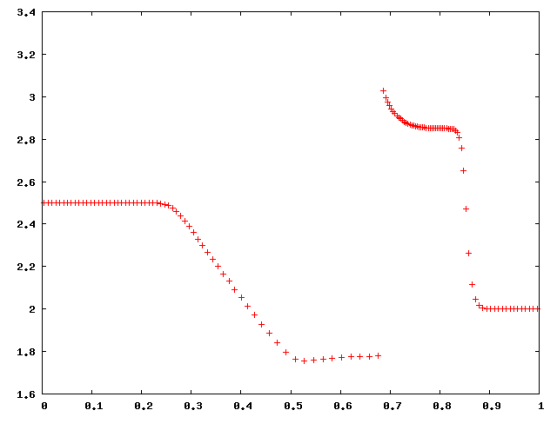

b)

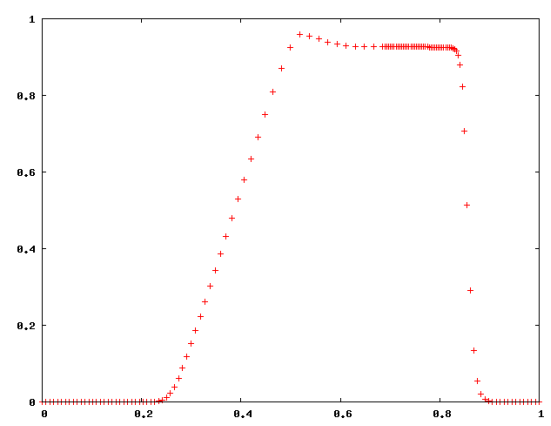

d)

Fig. 7. Pure cell single material Sod problem: 128 cells; a) - density, b) internal energy, c) pressure, d) velocity.

initial conditions are $u(x)=0, \rho(x)=1, \varepsilon(x)=2.5$ for $0 \geq x \geq 0.5$ and $\rho(x)=0.125, \varepsilon(x)=2.0$ for $0.5>x \geq 1$. The final time is $t=0.2$.

To obtain a reference solution for particular time and spatial discretizations (including artificial viscosity), numerical results are presented for Lagrangian calculations with pure cells. The computational mesh is comprised of $2^{k}$ cells (where $k$ is a parameter), with an equal number of cells on each side of the discontinuity.

In Fig. 7, plots for density, internal energy, pressure and velocity are presented for the $2^{7}=128$ pure cells calculation. Typical Lagrangian results obtained using pure cells are observed - in particular, an overshoot in internal energy near the contact is observed. It should not be expected that this feature, as well as other features of Lagrangian calculations with pure cells, will not be present in calculations with multimaterial cells.

Other important characteristics of the Lagrangian calculation with pure cells include the time evolution of parameters in the cells immediately to the left and right of the discontinuity, as shown in Fig. 8.

Several features can be seen in Fig. 8. First, in the history plot for pressure, it can be seen that the pressure in the left and right cells equilibrate after 


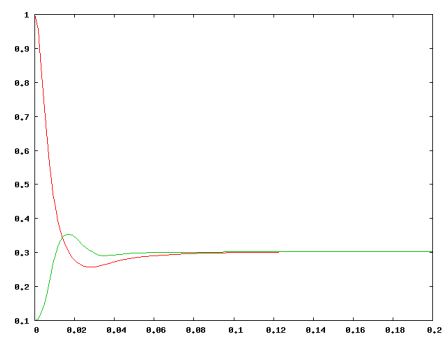

a)

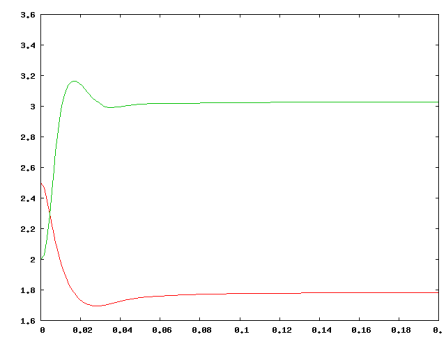

b)

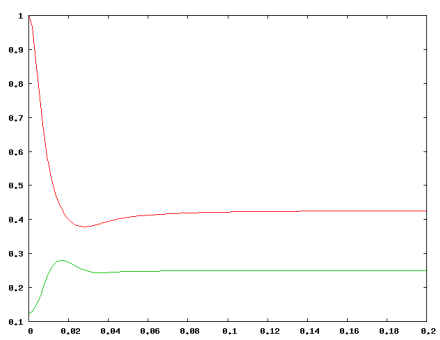

c)

Fig. 8. Pure cell single material Sod problem, 128 cells. Time history in the cells immediately to the right (green line) and left (red line) of the initial discontinuity: a) - pressure b) internal energy, c) density.

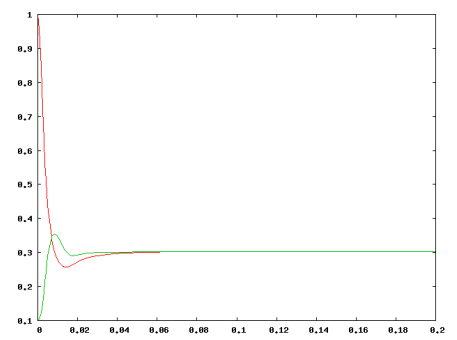

Fig. 9. Pure cell single material Sod problem, 256 cells: Time history of pressure in the cells on the right and left of initial discontinuity.

some transition time, which in this case is about $t=0.12$. The equilibration is expected, because the solution for this Riemann problem contains a contact: the contact will move with the fluid velocity, and therefore, it should form and remain between the same cells where the initial discontinuity was located. Because the feature is a contact, the pressure should be the same on left and right after some transition time when the solution structure is established. Another interesting observation is that the pressures in the left and right cells do not equilibrate in a smooth fashion: graphs of the pressures intersect each other before eventually equilibrating. It is also important to note that during the transition time, the internal energy reaches values that are significantly higher than the stationary value, which is itself higher than the exact value.

For 256 cells, the results are qualitatively similar. Only the time history of pressure equilibration is presented, see Fig. 9. It can be seen that the pressures equilibrate near $t=0.06$, that is, roughly twice as fast as the 128 cell case. Taking into account that the cells are half the size in this calculation indicates that the rate of equilibration is a characteristic of the test case, numerical method and the time step.

In order to obtain a comparable multimaterial simulation, the underlying mesh remains the same, but the position of the initial discontinuity is shifted by $0.5 \mathrm{~h}$ $\left(h=\left(x_{1}-x_{0}\right) / 2^{k}\right)$ with respect to the pure cell calculation so that it lies within cell $2^{k-1}+1$ (the first cell to the right of $x=0.5$ ) with $\alpha_{l}=\alpha_{r}=0.5$. 


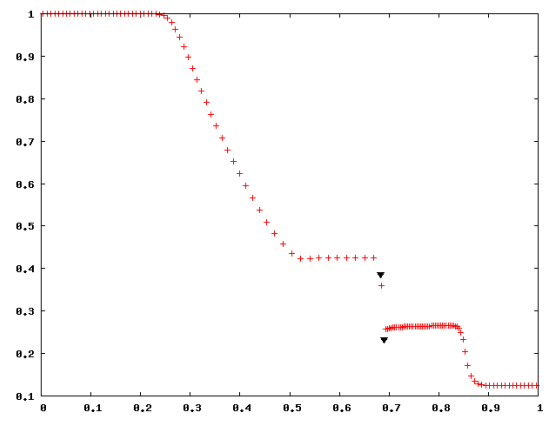

a)

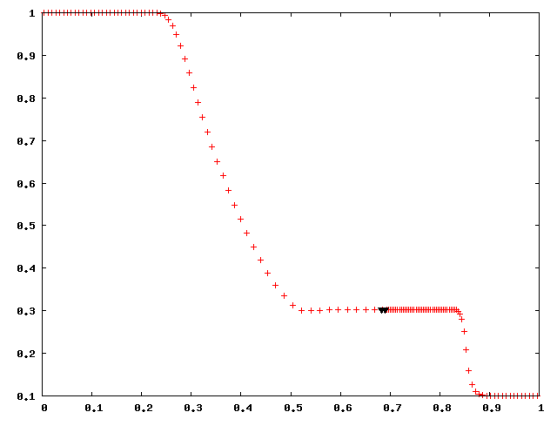

c)

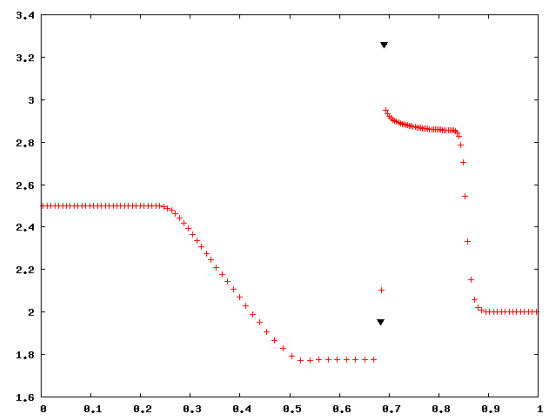

b)

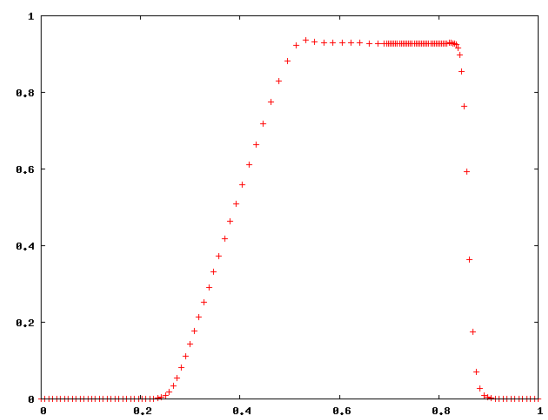

d)

Fig. 10. Pseudo two-material Sod problem, 128 cells. Calculation with one multimaterial cell with two identical materials. Tipton's closure model: red + cell averaged values, black solid triangles - material values in the multimaterial cell - a) - density, b) internal energy, c) pressure, d) velocity.

The material on the left of the interface is referred to as material \# 1 . The material on the right of the interface is referred to as material \# 2. Despite the two materials having the same equation of state, they are considered different materials (pseudo two-material) from the point of view of the closure model. In principle, this is a slightly different initial condition in comparison with the pure cell calculation because the discontinuity is in a different location. However, it corresponds to the same Riemann problem, therefore for qualitative analysis, the results may be compared with pure cell calculations.

To compare the Tipton and IA-SSD multimaterial calculations with the cellbased quantities presented for the pure cell calculation shown in Fig. 7, the volume averaged density, $\rho=\rho_{1} \alpha_{1}+\rho_{2} \alpha_{2}$, and mass averaged internal energy, $\varepsilon=\left(m_{1} / m\right) \varepsilon_{1}+\left(m_{2} / m\right) \varepsilon_{2}$, are presented in Figures 10 and 11 , where $m_{i}=$ $V_{i} \rho_{i}, i=1,2$ is the mass of material $i$ in the multimaterial cell, and $m=$ $m_{1}+m_{2}$ is the total mass. The material component densities, internal energies, and pressures computed by the equation of state are also shown in figures by black solid triangles. For Tipton's model, the common pressure used in the momentum equation is plotted, whereas for the IA-SSD model, the averaged pressure used in the momentum equation at the corrector stage is plotted. 


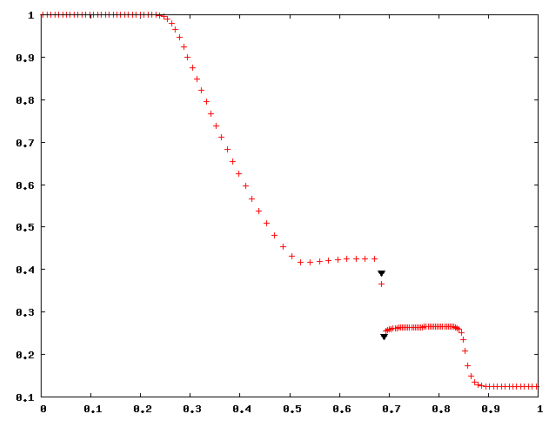

a)

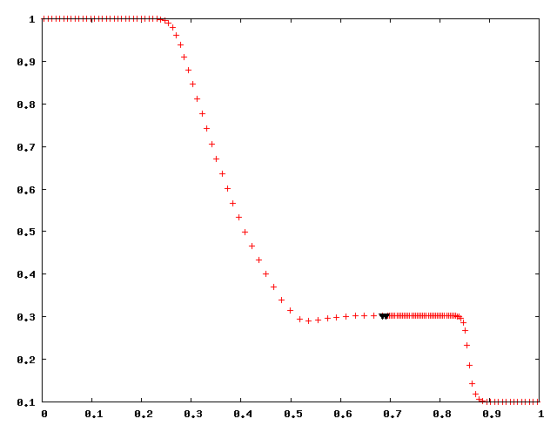

c)

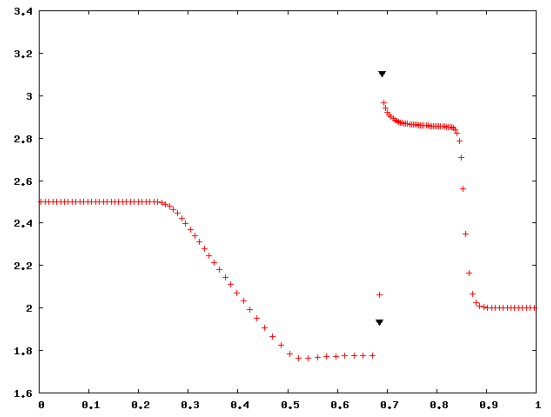

b)

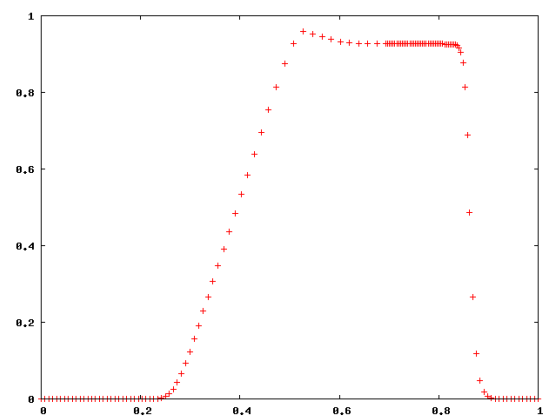

d)

Fig. 11. Pseudo two-material Sod problem, 128 cells. Calculation with one multimaterial cell with two identical materials. IA-SSD closure model: a) - density, b) internal energy, c) pressure, d) velocity.

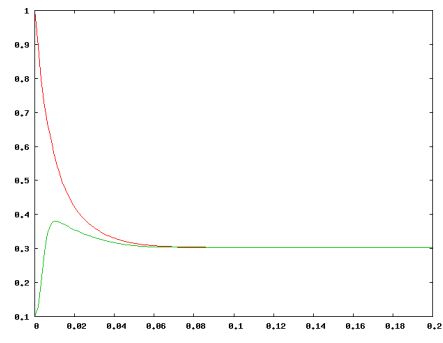

a)

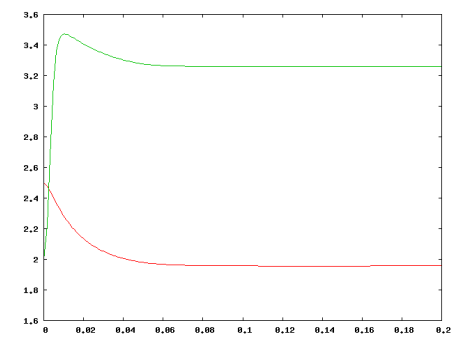

b)

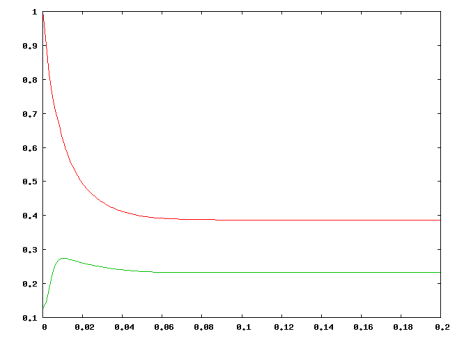

c)

Fig. 12. Pseudo two-material Sod problem, 128 cells. Calculation with one multimaterial cell with two identical materials, time history - material on the left of the discontinuity in the multimaterial cell (red line), material on the right of the discontinuity (green line). Tipton's closure model: a) - pressure b) internal energy, c) density.

From Figures 10 and 11 it is seen that the Tipton and IA-SSD solutions are qualitatively similar to the pure cell calculation. The most distinct feature is the significantly higher overshoot in material internal energy produced by the Tipton model on the right of the discontinuity - Fig. $10 \mathrm{~b}$ ). The overshoot in the IA-SSD calculation, $11 \mathrm{~b}$ ), is seen to be significantly smaller than the one observed in the Tipton solution. 


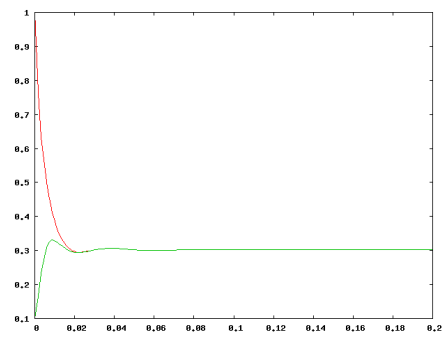

a)

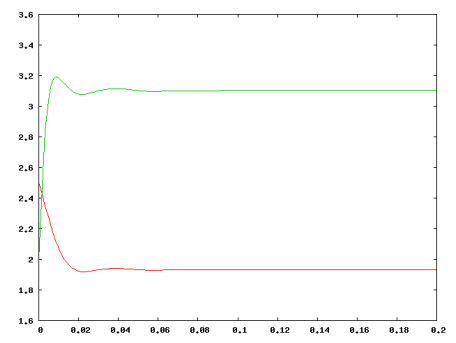

b)

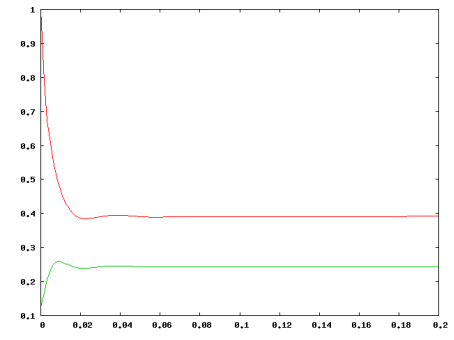

c)

Fig. 13. Pseudo two-material Sod problem, 128 cells. Calculation with one multimaterial cell with two identical materials, time history. IA-SSD closure model: a) pressure b) internal energy, c) density

Time history plots of material values in the multimaterial cell are shown in Figures 12 and 13 for the Tipton and IA-SSD calculations respectively. It can be noted that the time at which the Tipton material pressures equilibrate is roughly the same as for the pure cell calculation despite the effective mesh size near the discontinuity being halved. Also, the pressure graphs do not intersect each other as in the case of the pure cell calculation. The IA-SSD pressure equilibration process differs from Tipton's model. Pressures equilibrate at about $t=0.02$. After this time, material pressures are equal in both materials but keep changing and reach "exact" value at $t=0.06$ as may be expected from analysis of the pure cell calculation. Note that in the IA-SSD closure model the rate of equilibration can be controlled. This is demonstrated in Section 8.1.4, however, in this test case the default parameter values are used and the constraints in the IA-SSD model are not active (the limiter is equal to unity at all times).

\subsubsection{Volume fraction sensitivity - importance of limiter}

In previous Sections, all problems were considered with equal volume fractions, $\alpha_{l}=\alpha_{r}=0.5$. In real calculations, situations may arise where the initial values of the volume fractions in the multimaterial cells can vary dramatically from very small to very large. The simplest example is when a material with a circular shape is "painted" over a square mesh. The closure model's treatment of such situations is therefore of significance.

To examine this scenario, the standard single material Sod problem (as previously described) is performed with the modification where the interface within the multimaterial cell is shifted to the right by $0.9 \mathrm{~h}$. The volume fractions of the left and right materials are then $\alpha_{l}=0.9$, and $\alpha_{r}=0.1$.

For this problem, profiles of the internal energy are presented along with the time history for pressure and internal energy. The results for Tipton's model are presented in Fig. 14, where the main feature that can be seen is a large 


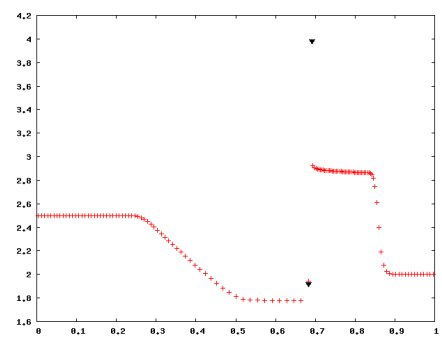

a)

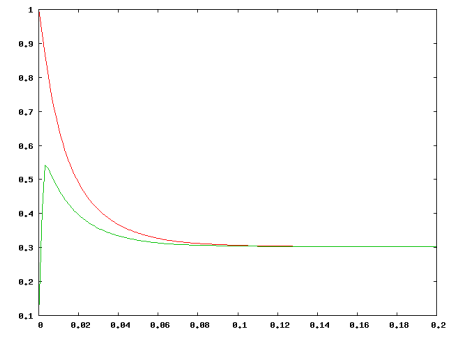

b)

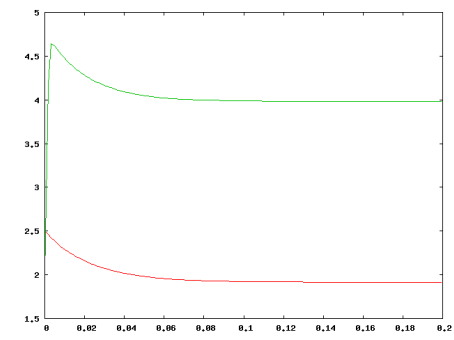

c)

Fig. 14. Pseudo two-material Sod problem with $\alpha_{L}=0.9$. Tipton's model, a) Profile of the internal energy; Time history: b) pressure; c) internal energy.

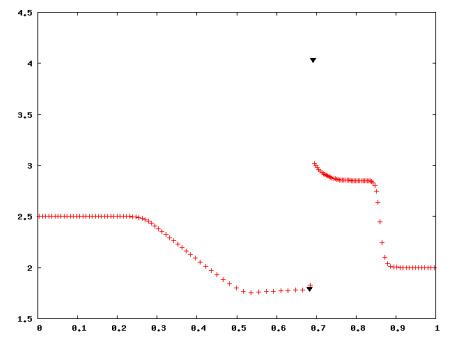

Fig. 15. Pseudo two-material Sod problem with $\alpha_{L}=0.9$. Tipton's model with zero characteristic length - internal energy profile.

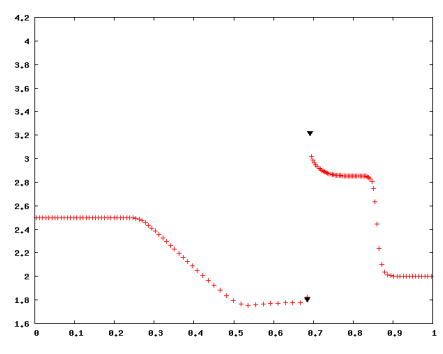

a)

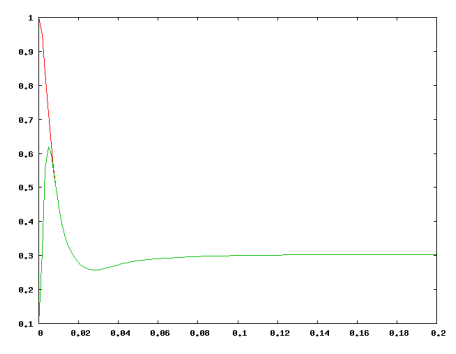

b)

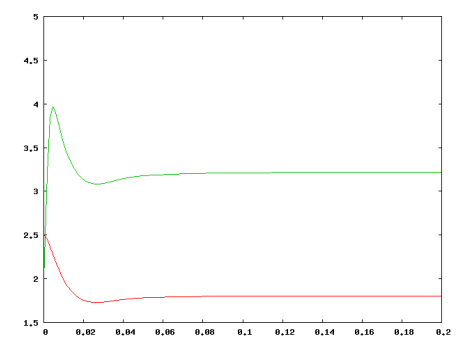

c)

Fig. 16. Pseudo two-material Sod problem with $\alpha_{L}=0.9$. IA-SSD model, a) internal energy profile; Time history: b) pressure; c) internal energy.

overshoot in the internal energy. Setting the characteristic length in Tipton's model to zero (instantaneous equilibration) does not fix this problem, but rather, exaggerates it, see Fig. 15.

The results for the IA-SSD model are presented in Fig. 16 with the overshoot in the internal energy for IA-SSD model being smaller than for Tipton's model. History plots are qualitatively similar to the case when the initial volume fractions were equal.

Note that in the previous multimaterial calculations where the initial volume fractions were equal, the constraints in the IA-SSD closure model were not active so that the limiter was equal to one. In the case of a small volume 


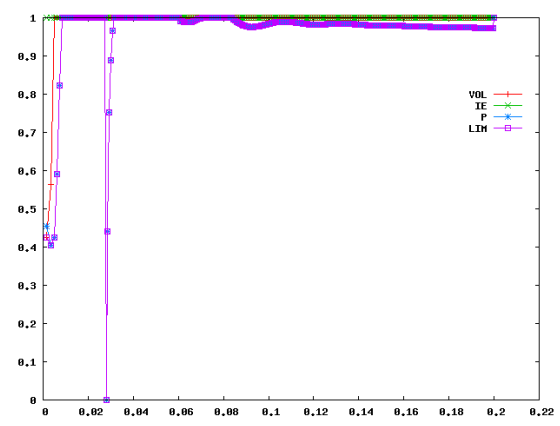

Fig. 17. Pseudo two-material Sod problem with $\alpha_{L}=0.9$. IA-SSD model. Time history for limiters: VOL - volume, IE - internal energy, P - pressure, LIM - overall limiter.

fraction for the right material, the constraints are active. The initial pressure of the left material is higher than the pressure of the right material, and on the sub-scale stage of IA-SSD, the interface between the materials may move significantly to the right which would result in a negative volume for the right material. Therefore, the change in the volume during the sub-scale stage needs to be limited. The time histories for limiters related to the volume, internal energy and pressure equilibration constraints in the IA-SSD model are presented in Fig. 17. It can be seen that with the exception of the first time step, when the overall limiter is defined by the volume constraint, the overall limiter coincides with the limiter determined by the pressure equilibration constraint. The limiter reaches a minimal value at a time around $t=0.025$, which is close to the moment when material pressures are equilibrated and are starting to grow before reaching a value close to the "exact" value. If in this particular calculation the limiter is set to equal one, the volume of the right material will become negative after the first time step.

\subsubsection{Two-material calculations}

\section{Two-material Sod problem}

A two material version of the Sod problem is performed. The computational domain is a segment with bounds $[0: 1]$. The materials are ideal gases: $p=$ $(\gamma-1) \rho \varepsilon$. The material on the left of $x=0.5$ uses $\gamma=2$, and on the right $\gamma=$ 1.4. The boundary conditions are $u(0)=u(1)=0$. The initial conditions are $u(x)=0, \rho(x)=1, \varepsilon(x)=2.5$ for $0 \geq x \geq 0.5$ and $\rho(x)=0.125, \varepsilon(x)=2.0$ for $0.5>x \geq 1$. The final time is $t=0.2$. The underlying mesh and location of the multimaterial cell are the same as for case of the single material Sod problem.

Figure 18 presents the internal energy profile and time histories of pressure and internal energy for the two material pure cell calculation. 


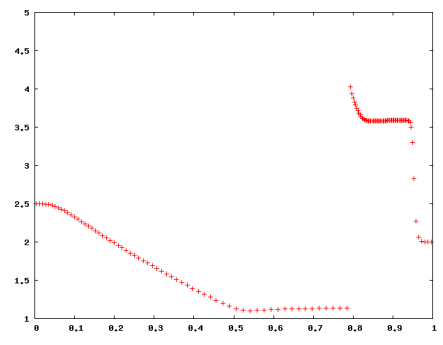

a)

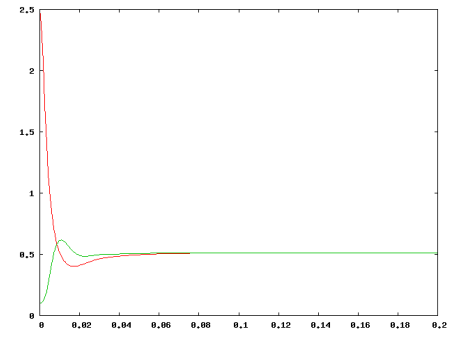

b)

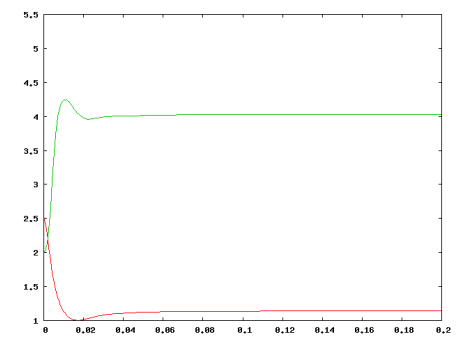

c)

Fig. 18. Two material Sod problem - pure cell calculation: a) Profile of internal energy; Time history: b) pressure, c) internal energy.

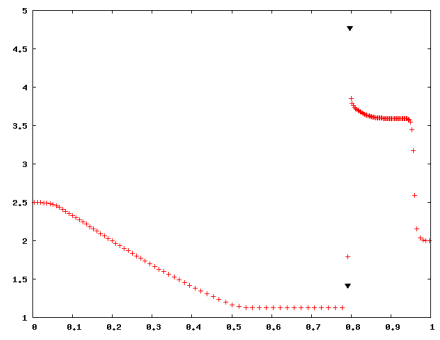

a)

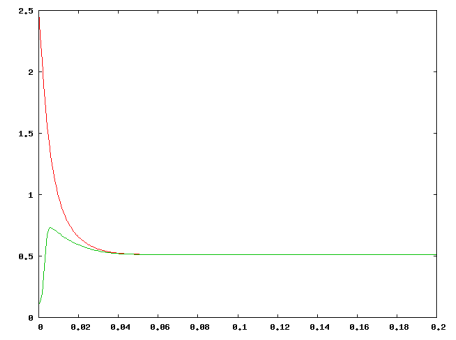

b)

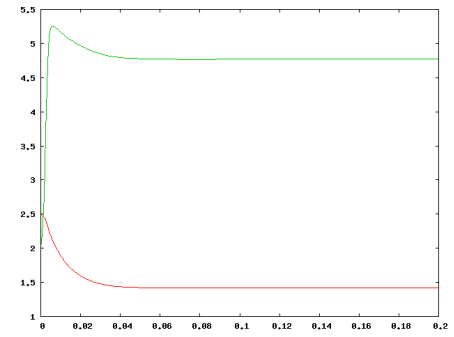

c)

Fig. 19. Two material Sod problem - Tipton's model: a) Profile of internal energy; Time history: b) pressure, c) internal energy.

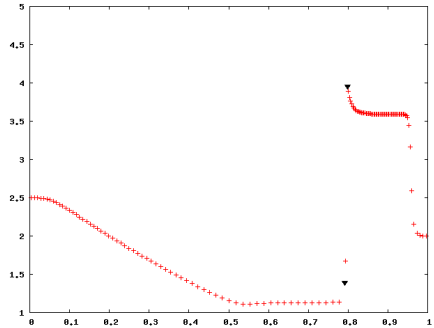

a)

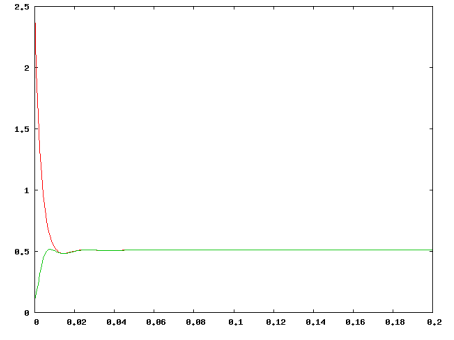

b)

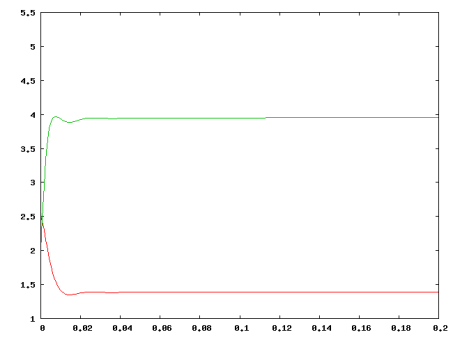

c)

Fig. 20. Two material Sod problem - IA-SSD model: a) Profile of internal energy; Time history: b) pressure, c) internal energy.

For multimaterial calculations, the interface is shifted by $0.5 h$ so that $\alpha_{l}=$ $\alpha_{r}=0.5$. Internal energy profiles and time histories for pressure and internal energy are shown in Figures 19 and 20 for the Tipton and IA-SSD models. The qualitative conclusions for the two-material Sod problem are the same as for the single material Sod problem - the IA-SSD closure model provides a smaller overshoot in internal energy compared to Tipton's closure model.

\section{Water-air shock tube problem}

Next, the "water-air" problem (see, for example, $[7,48]$ ) is considered. The 


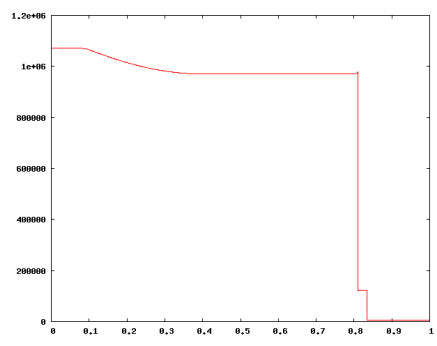

a)

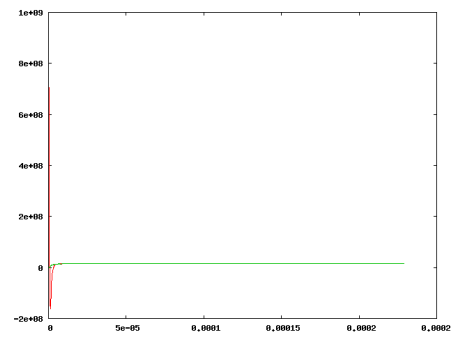

b)

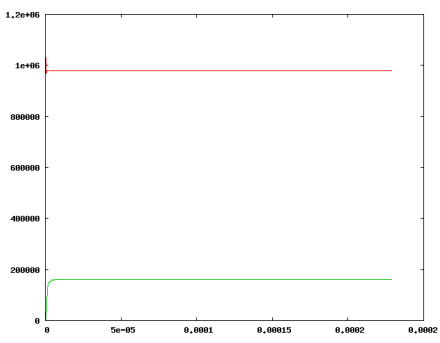

c)

Fig. 21. Water-air problem - pure cell computation. a) Profile of internal energy; Time history b) pressure; c) internal energy.

computational domain is $[0: 1]$, with both boundaries defined as stationary walls. The left material $(x<0.7)$ represents water, which is modeled using the stiffened-gas equation of state

$$
p=(\gamma-1) \rho \varepsilon-\gamma \pi ; \quad \gamma=4.4, \pi=6 \times 10^{8} .
$$

The initial state of the water is $p=10^{9}, \rho=10^{3}, u=0$. The right material $(x \geq 0.7)$ is air, which is an ideal gas: $p=(\gamma-1) \rho \varepsilon ; \gamma=1.4$. The initial state of the air is $p=10^{5}, \rho=50, u=0$. The final time is $t=2.29 \times 10^{-4}$. This test case represents a more severe problem compared to those previously considered, both in terms of the magnitude of the discontinuity and equation of state used for the water component.

To be consistent with results presented for this problem in other papers, an initially uniform mesh with 1000 cells is used such that mesh vertex \# 700 coincides with the discontinuity. For computations including a multimaterial cell, the multimaterial cell is located to the right of the interface at $x=0.7$ (cell \# 701).

Figure 21 presents a profile of internal energy and the time histories for pressure and internal energy for the pure cell water-air computation. The main feature in this case is that prior to equilibration, the pressure of the water attains negative values. Close examination of the time history of the pressure equilibration reveals additional features of note. Magnified views of the pressure history for different periods of time are presented in Fig. 22.

It can be seen in Fig. 22 that numerous oscillations exist. It is not expected that such oscillations will not also feature in the multimaterial computations.

Figure 23 presents the internal energy profile and the time histories for pressure and internal energy for the simulation using Tipton's model. It can be observed that the pressure remains positive during the transition time. Figure 24 presents magnified views of the pressure history for time periods corresponding to those shown for the pure cell calculation. The equilibration process is 


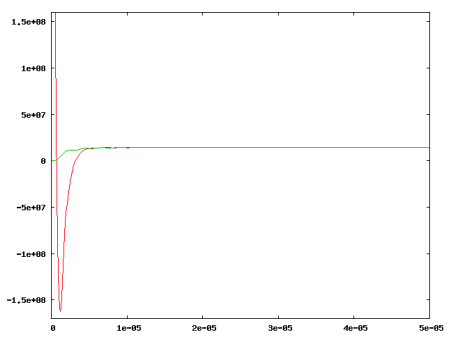

a)

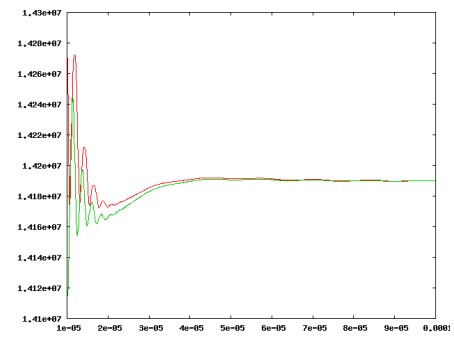

b)

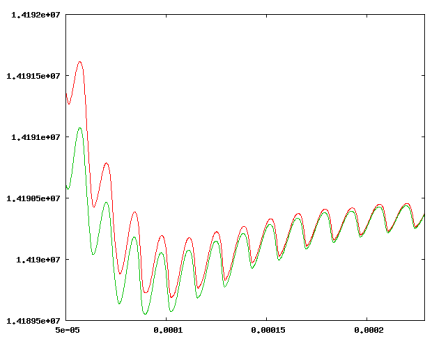

c)

Fig. 22. Water-air problem - pure cell computation. Fragments of pressure equilibration history: a) close to $t=0, \mathrm{~b}$ ) beginning of the tail, c) end of tail. Note that the scale differs for each plot.

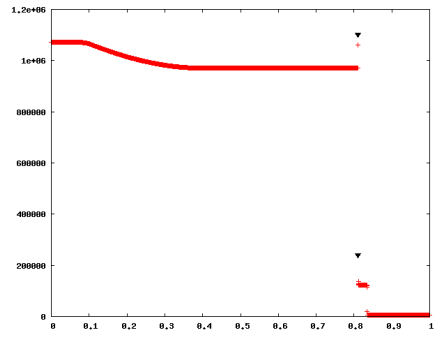

a)

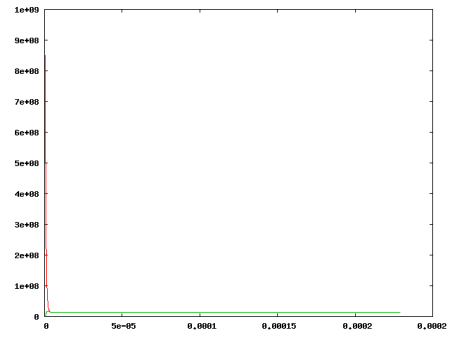

b)

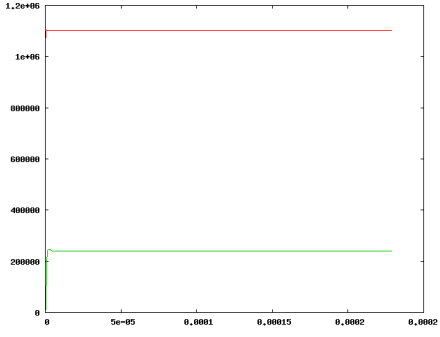

c)

Fig. 23. Water-air problem - multimaterial cell computation - Tipton's model. a) Profile of internal energy; Time history: b) pressure; c) internal energy.

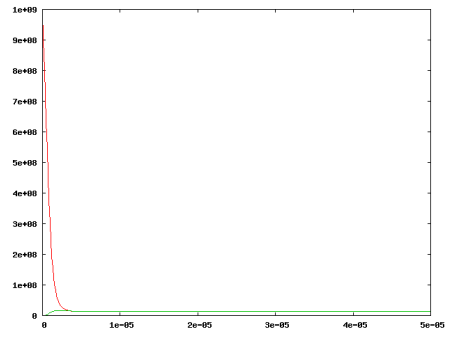

a)

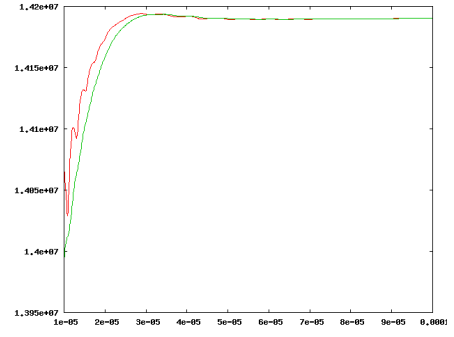

b)

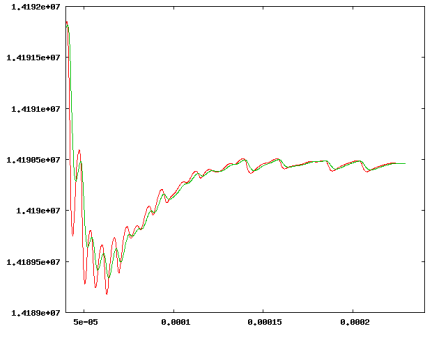

c)

Fig. 24. Water-air problem - multimaterial cell computation - Tipton's model. Fragments of pressure equilibration history: a) close to $t=0, \mathrm{~b}$ ) beginning of the tail, c) end of tail

smoother, and at the final time the pressures are less equilibrated than for the pure cell calculation.

Figure 25 presents a profile of internal energy and the time histories for pressure and internal energy for the IA-SSD calculation. It can be noted that in this simulation, negative values of water pressure are introduced during the equilibration process. These values are smaller in magnitude compared to the pure cell calculation. Also, the IA-SSD internal energy value for the air differs 


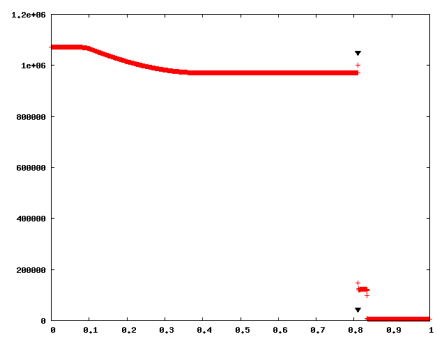

a)

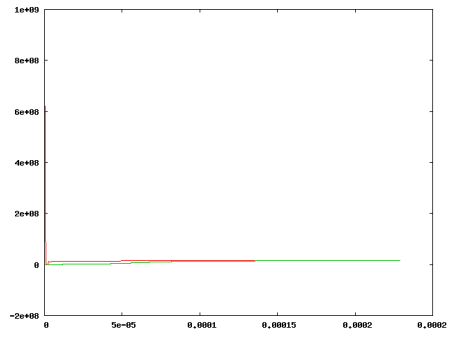

b)

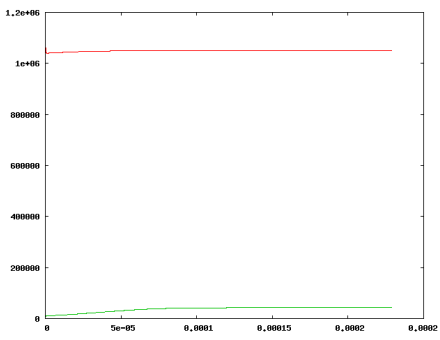

c)

Fig. 25. Water-air problem - multimaterial cell computation - IA-SSD model. a) Profile of internal energy; Time history: b) pressure; c) internal energy.

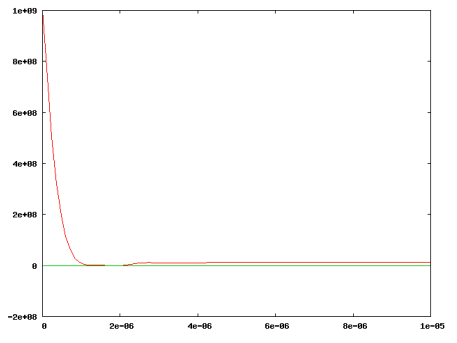

a)

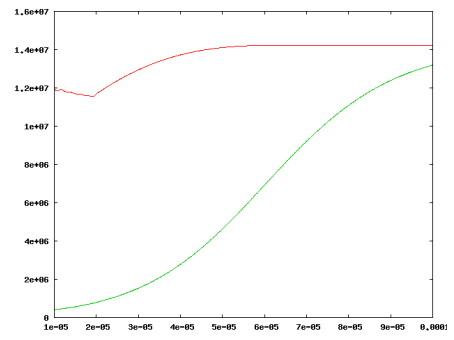

b)

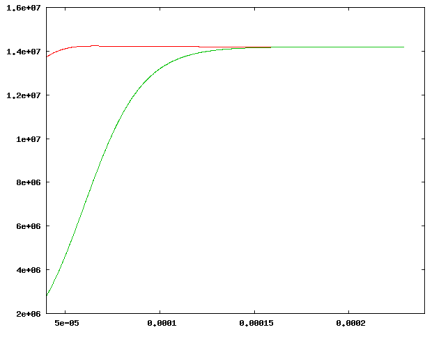

c)

Fig. 26. Water-air problem - multimaterial cell computation - IA-SSD model. Fragments of pressure equilibration history: a) close to $t=0, \mathrm{~b}$ ) beginning of the tail, c) end of tail

significantly from the value obtained by Tipton's model.

Figure 26 presents magnified views of the pressure history for time periods corresponding the pure and multimaterial Tipton calculations - it can be seen that the equilibration process is much smoother.

\subsubsection{Controlling equilibration in IA-SSD}

In the previous calculations using the IA-SSD model, the value $\kappa_{i}=0.99$ was used for the parameters in the pressure equilibration constraint, and a value $\kappa_{\text {bot }}=0.5$ for the parameter in volume constraints.

One reason why the IA-SSD model is presented as a "framework" is because the theory for optimal choice of parameters in the constraints remains to be developed. However, a demonstration of how the parameter in the pressure equilibration constraints affects the equilibration process is useful.

The two material Sod problem is presented for the case when the pressure equilibration parameter is reduced from $\kappa_{i}=0.99$ to $\kappa_{i}=0.5$. Interpreting the pressure constraints, it follows that the material pressures will be allowed 


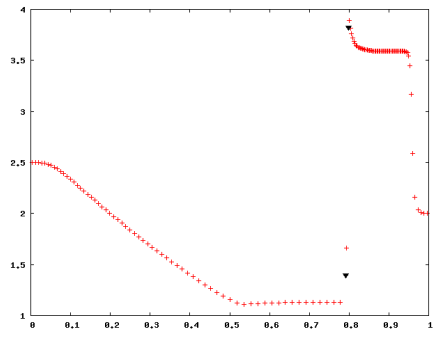

a)

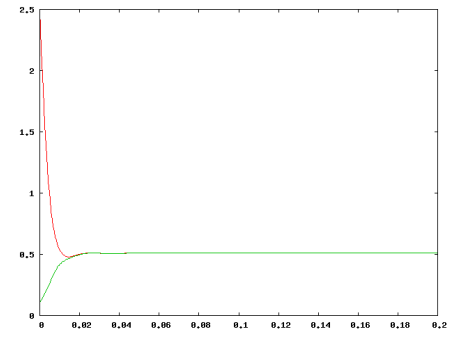

b)

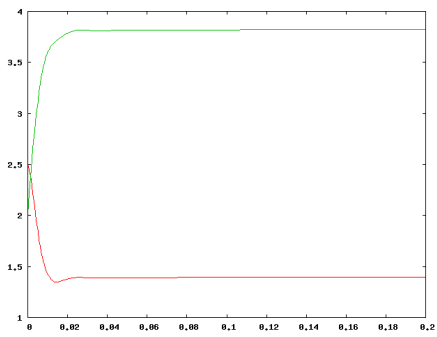

c)

Fig. 27. Two material Sod problem - IA-SSD model with $\kappa_{i}=0.5$ : a) Profile of internal energy; Time history: b) pressure, c) internal energy.

to approach each other at a lower rate in each time step as $\kappa_{i}$ goes to zero.

Figure 27 shows a profile of internal energy and the time histories for pressure and internal energy for this value of the parameter. From a comparison of Figures 20 and 27, it can be concluded that an increase in the pressure equilibration parameters leads to longer (as expected) pressure equilibration time. Also, the process of equilibration is smoother, and the overshoot in internal energy is reduced.

\subsubsection{One-dimensional problems with three materials}

\section{Two-material Sod problem with three-material cell}

The two material Sod shock problem is performed with parameters as previously described, however, the problem now features a three-material cell. Within this cell, two materials will have identical properties. Two cases, defined by the relative values of the material states, are considered - identified as 'High-High-Low' (HHL) and 'High-Low-Low' (HLL). In the HHL case, the left and center materials correspond to the initial left state in the Sod problem, and the material on the right corresponds to the right state. In the HLL case the left material corresponds to the left state and the center and right materials correspond to right state. In both cases, all materials have equal volume fractions $\alpha=1 / 3$.

\section{HHL case}

Figures 28 and 29 present profiles for the Tipton and IA-SSD models, with both methods performing similarly to the two-material cell simulation (including the overshoot in the Tipton case).

Figures 30 and 31 present time histories of variables. Qualitative differences between the Tipton and IA-SSD histories can be seen. In both methods, the pressures of all materials equilibrate. In the Tipton solution, the materials with 

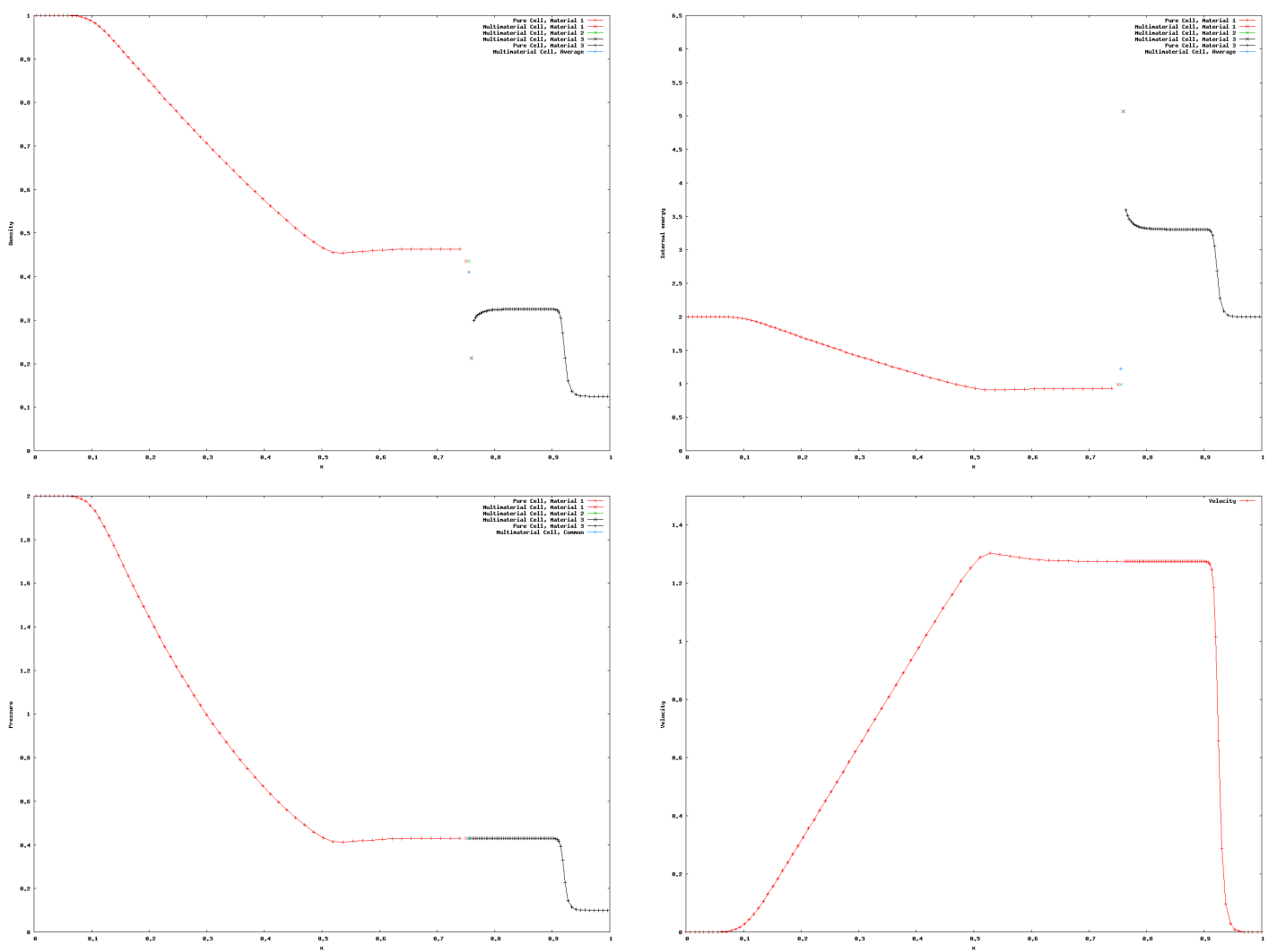

Fig. 28. Two material Sod problem with three material multimaterial cell - HHL case, Tipton's model. Top left: density, top right: internal energy, bottom left: pressure, bottom right: velocity.

initially identical values evolve identically so that the history plots for these parameters coincide. In the IA-SSD simulation however, the pressure of the middle material moves toward equilibrium first. This behavior is anticipated from the sub-scale dynamics because the interface between the middle and right materials will move first due to the difference in the pressure on the left and the right side of this interface. The interface between the left and center materials should begin to move at a later time once the pressure in the center material drops.

Figure 32 presents time histories of limiters for the HHL case. In this case there are two interfaces, with two corresponding limiters. It can be seen that only the limiter controlling the interaction between the middle and right materials is active, and the limiter corresponding to the interface between the left and center materials is not active (it is equal to one). This is explained because the main interactions occur between the materials which have initially different pressures.

$\underline{H L L}$ case

Profiles for the Tipton and IA-SSD solutions are shown in Figures 33 and 34. 

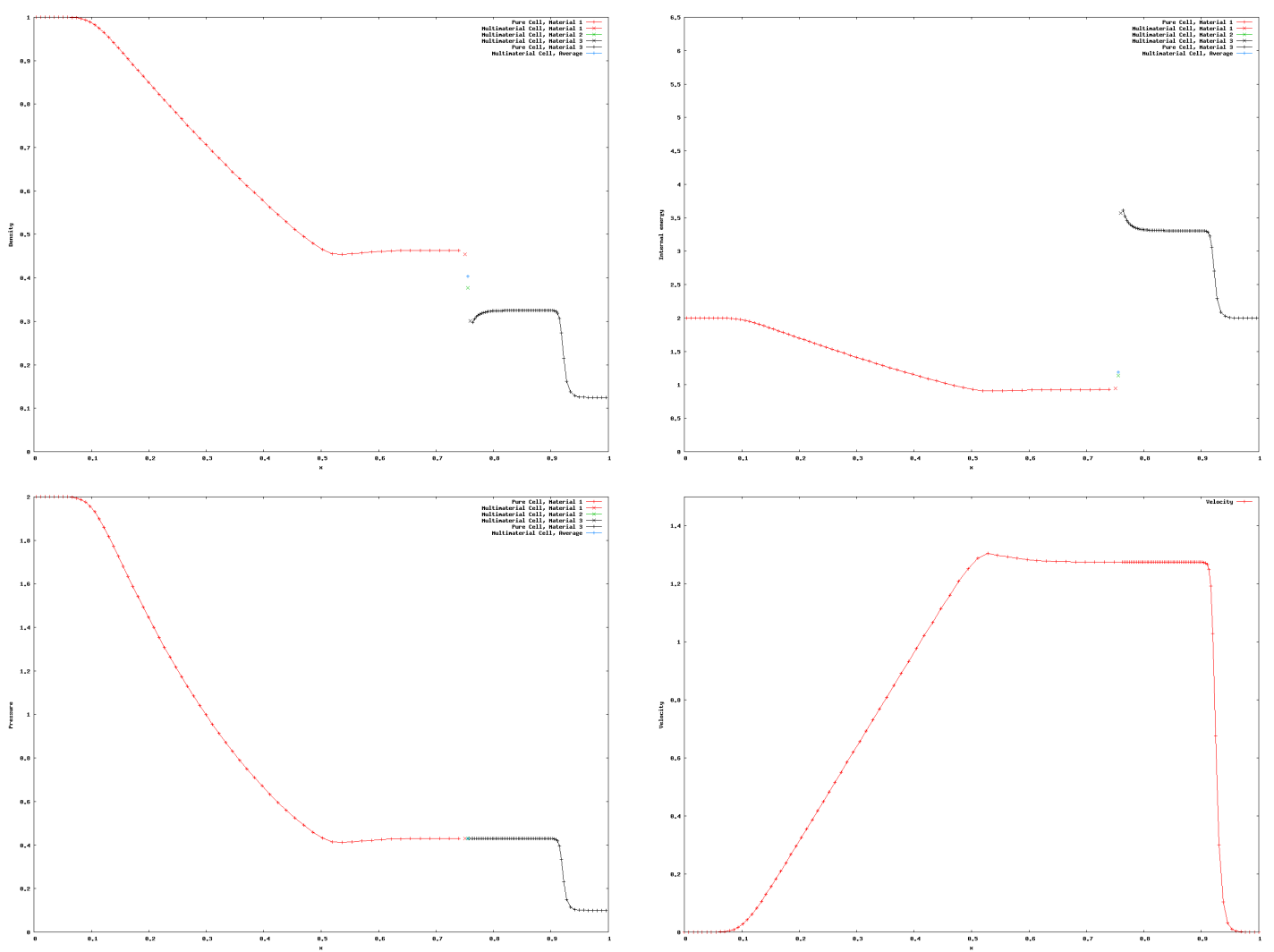

Fig. 29. Two material Sod problem with three material multimaterial cell- HHL case, IA-SSD model. Top left: density, top right: internal energy, bottom left: pressure, bottom right: velocity.
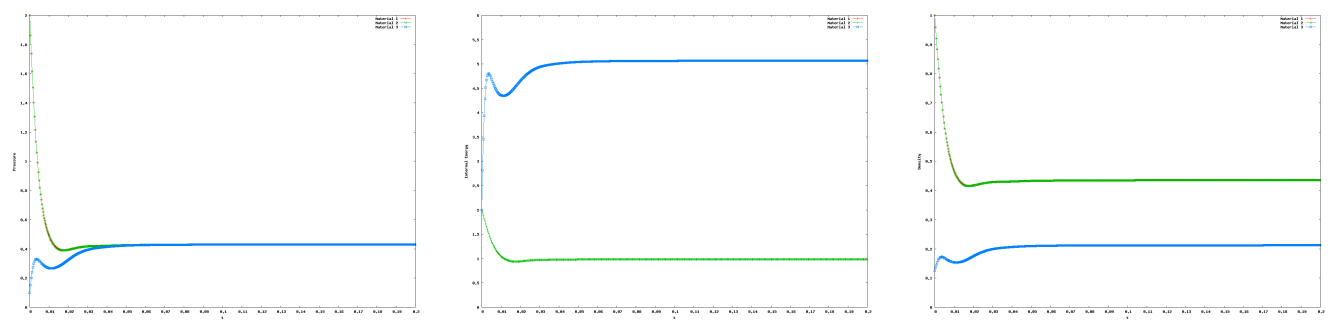

Fig. 30. Two material Sod problem with three material multimaterial cell - HHL case, Tipton's model. Time history: left - pressure, center - internal energy, right density.

Figures 35 and 36 show the time histories for the two methods. As in the HHL case for the IA-SSD method, it can be seen that the pressure equilibration first starts in the middle material for the same reason - the pressures either side of this interface are different.

Figure 37 presents a time history of IA-SSD limiters for the HLL case. The behavior of the limiters for the HLL case is quite different from the HHL case. This difference arises because in the HLL case, both the H-L and L-L interfaces within the cell are physically able to move to the right, therefore the limiter 

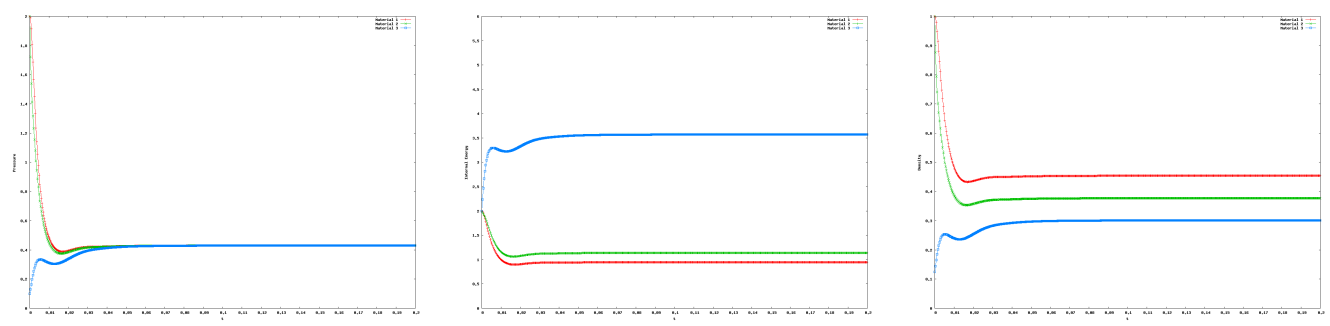

Fig. 31. Two material Sod problem with three material multimaterial cell - HHL case, IA-SSD model. Time history: left - pressure, center - internal energy, right density.

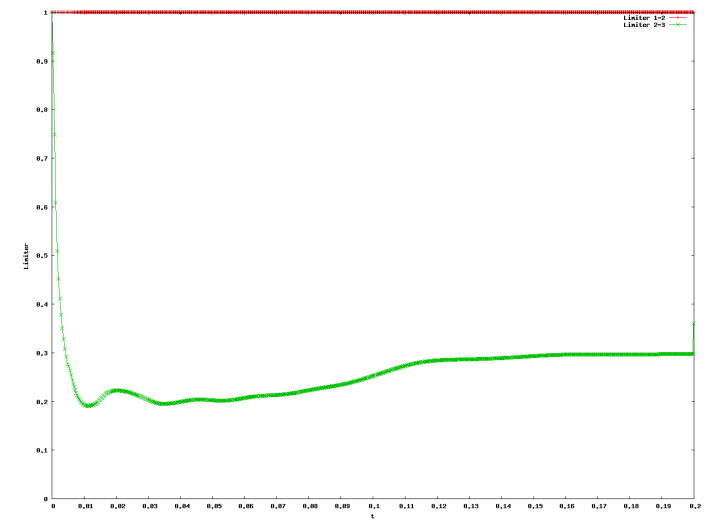

Fig. 32. Two material Sod problem with three material multimaterial cell - HHL case, IA-SSD model. Time histories of limiters.

may remain at unity. However in the HHL case the H-L interface is driven closer to the cell boundary on the right which cannot move. Therefore the limiter corresponding to this interface must restrict the movement. It can also be mentioned that in the HLL case this limiter is active in several short time periods when the pressures are close to equilibrium; in the HHL the limiter is active at all times.

\section{Single isolated three-material cell}

The pressure equilibrium behavior is now examined in a single cell. The cell has solid boundaries so that the effects of sub-scale interactions are isolated. A number of combinations of material configuration are considered depending upon whether materials have either high-, medium- or low-value properties. The material properties allow 27 possible configurations, but with symmetry and similarities, there are only 5 distinct cases. In all calculations, the computational domain is one cell defined over $[0,0.1]$. The initial volume fractions for all cases are $\alpha=1 / 3$, and the final time is $t=0.05$. The 'high' valued material has properties $\rho_{H}=1.0, p_{H}=2.0, \gamma_{H}=2.0$. The 'medium' valued material has properties $\rho_{M}=0.5625, p_{M}=1.05, \gamma_{M}=1.9333$. The 'low' valued material has properties $\rho_{L}=0.125, p_{L}=0.1, \gamma_{L}=1.4$. 

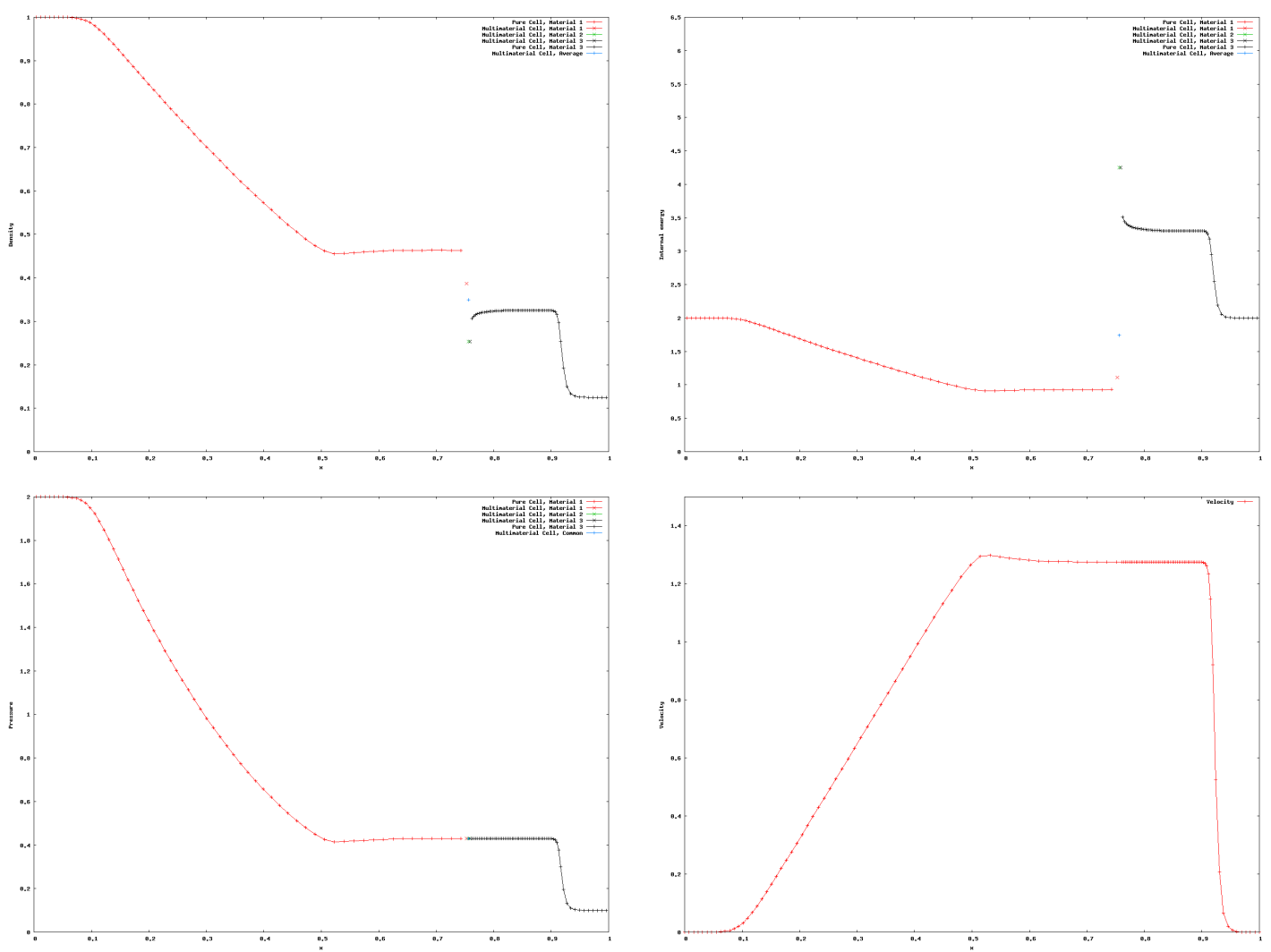

Fig. 33. Two material Sod problem with three material multimaterial cell - HLL case, Tipton's model. Top left: density, top right: internal energy, bottom left: pressure, bottom right: velocity.

Note that the cell is isolated and the end points do not move. For this reason, the momentum equation plays no role in the equilibration process. The pressure equilibration process for the Tipton and IA-SSD models are different, and therefore it cannot be expected that for the same initial conditions, the equilibrium state will be the same for both models. Also, it should be noted that in general there is an infinite number of stationary states for both models. Any state in which all three pressures are the same is a stationary state for both models.

\section{$\underline{H H L}$ case}

In this case the materials in the cell are configured as high-high-low.

The pressure evolutions of the three materials for the HHL case are shown in Figure 38 for the Tipton and IA-SSD models. These plots are similar to those shown for the HHL case of the Sod problem previously presented.

\section{$\underline{H L L}$ case}

In this case the materials in the cell are configured as high-low-low. 

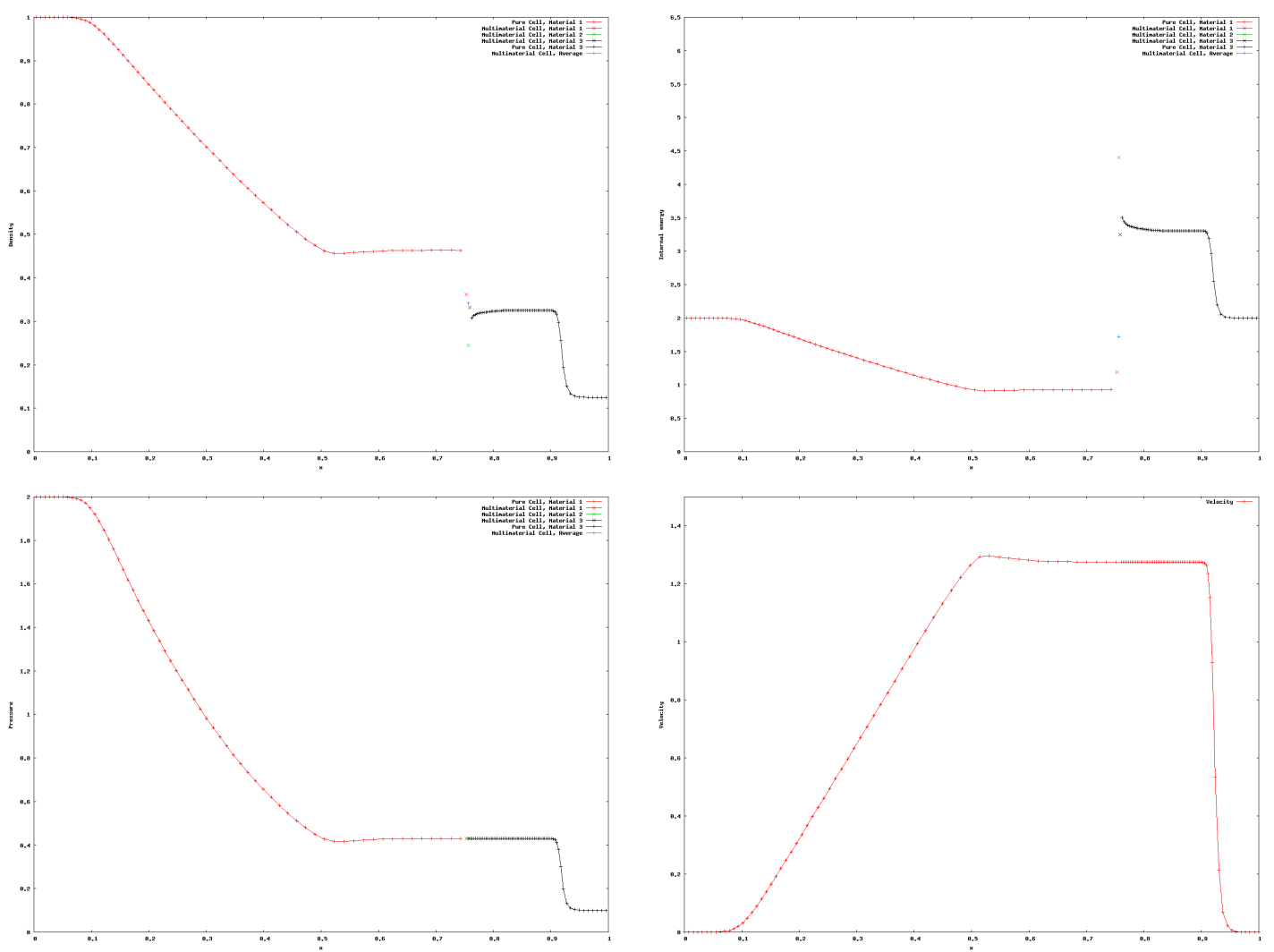

Fig. 34. Two material Sod problem with three material multimaterial cell - HLL case, IA-SSD model. Top left: density, top right: internal energy, bottom left: pressure, bottom right: velocity.
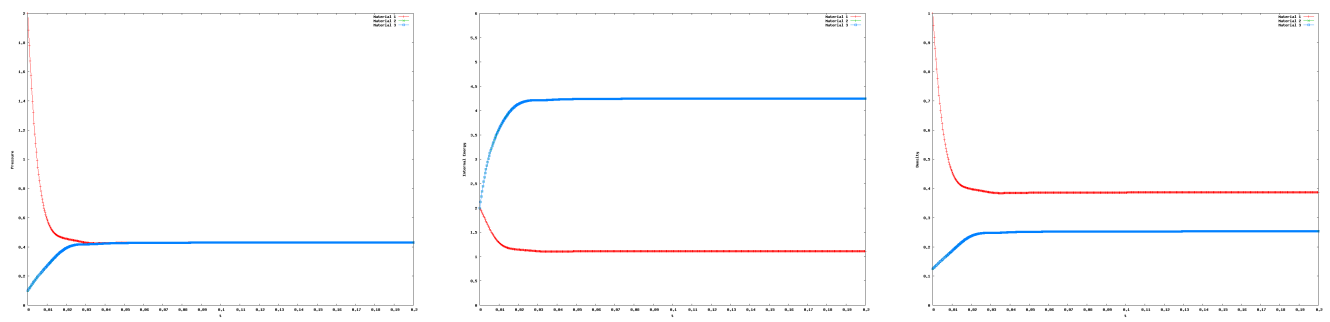

Fig. 35. Two material Sod problem with three material multimaterial cell - HLL case, Tipton's model. Time history: left - pressure, center - internal energy, right density.

The pressure evolutions of the three materials for the HLL case are shown in Figure 39 for the Tipton and IA-SSD models. Again, these plots are similar to those for the HLL case of the Sod problem presented before.

\section{$\underline{H L H \text { case }}$}

In this case the materials in the cell are configured as high-low-high.

The pressure evolutions of the three materials for the HLH case are shown in Figure 40 for the Tipton and IA-SSD models. Because of the symmetry in the 

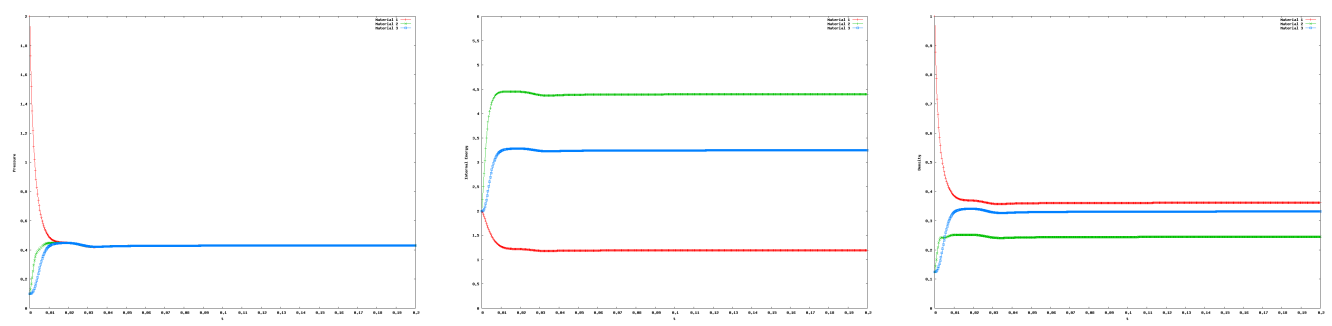

Fig. 36. Two material Sod problem with three material multimaterial cell - HLL case, IA-SSD model. Time history: left - pressure, center - internal energy, right density.

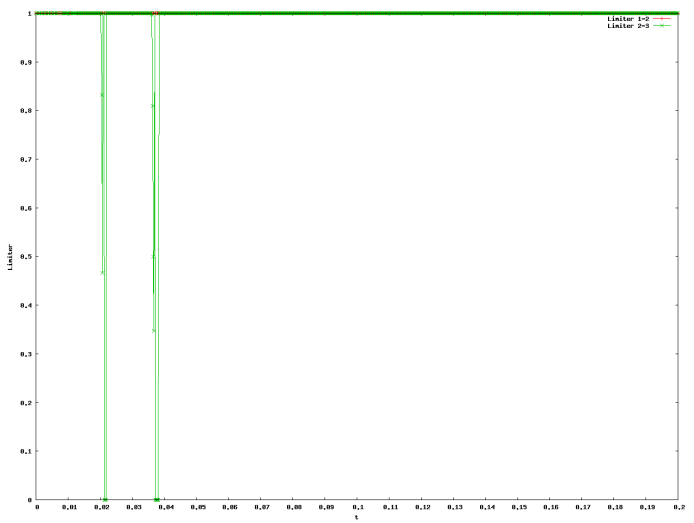

Fig. 37. Two material Sod problem with three material multimaterial cell - HLL case, IA-SSD model. Time histories of limiters.
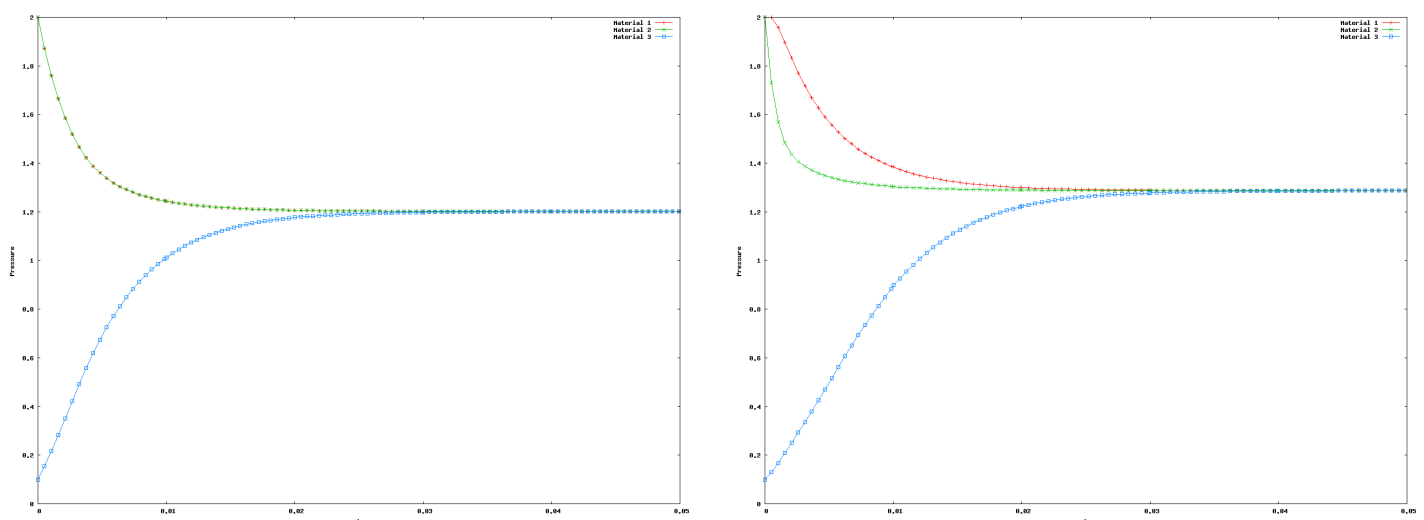

Fig. 38. Time histories of pressure, three-material one-cell - HHL. Left: Tipton, right: IA-SSD.

HLH case, the plots of the left and right materials coincide with each other for both models. It is interesting to note that the Tipton and IA-SSD models give visibly different equilibrated pressures.

\section{$\underline{\text { LHL case }}$}

In this case the materials in the cell are configured as low-high-low. 

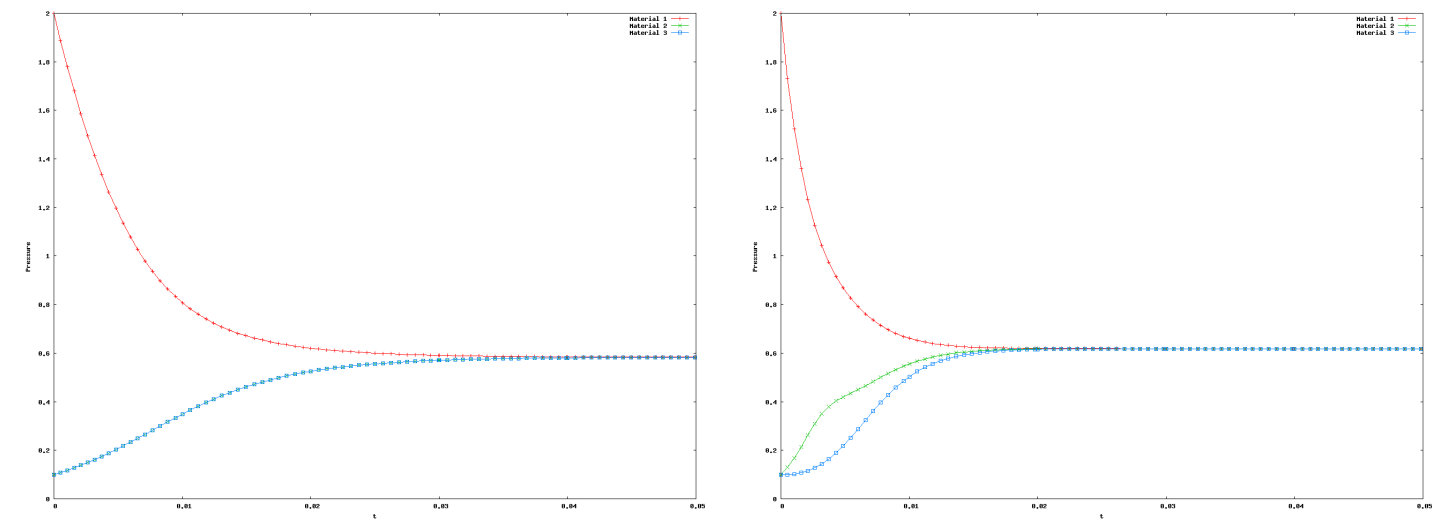

Fig. 39. Time histories of pressure, three-material one-cell - HLL. Left: Tipton, right: IA-SSD.
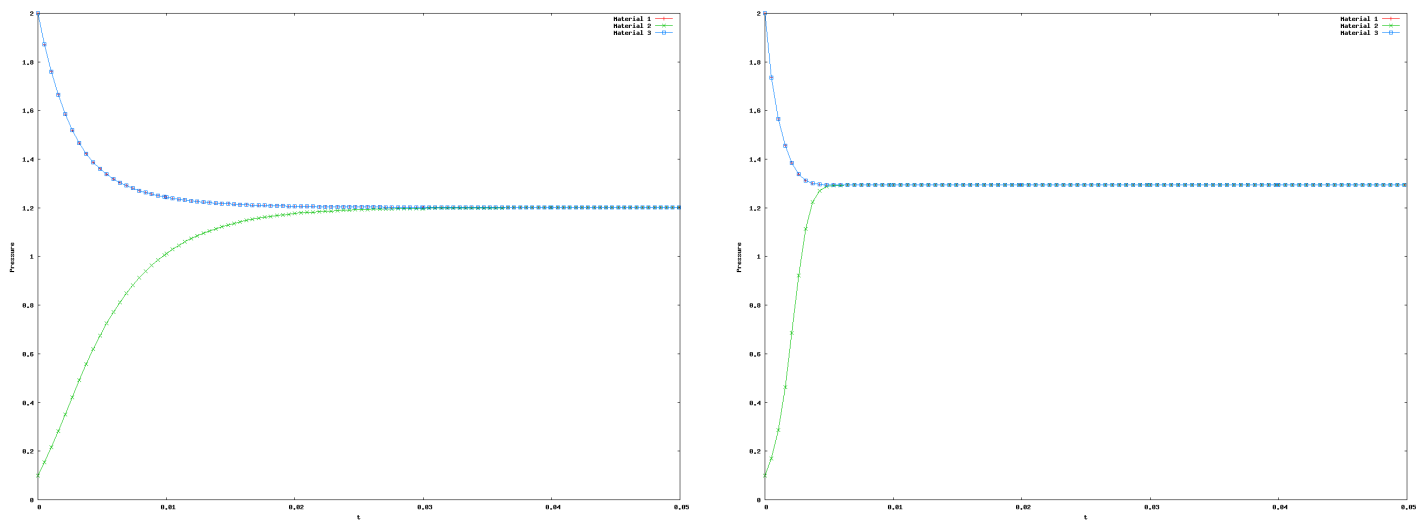

Fig. 40. Time histories of pressure, three-material one-cell - HLH. Left: Tipton, right: IA-SSD.
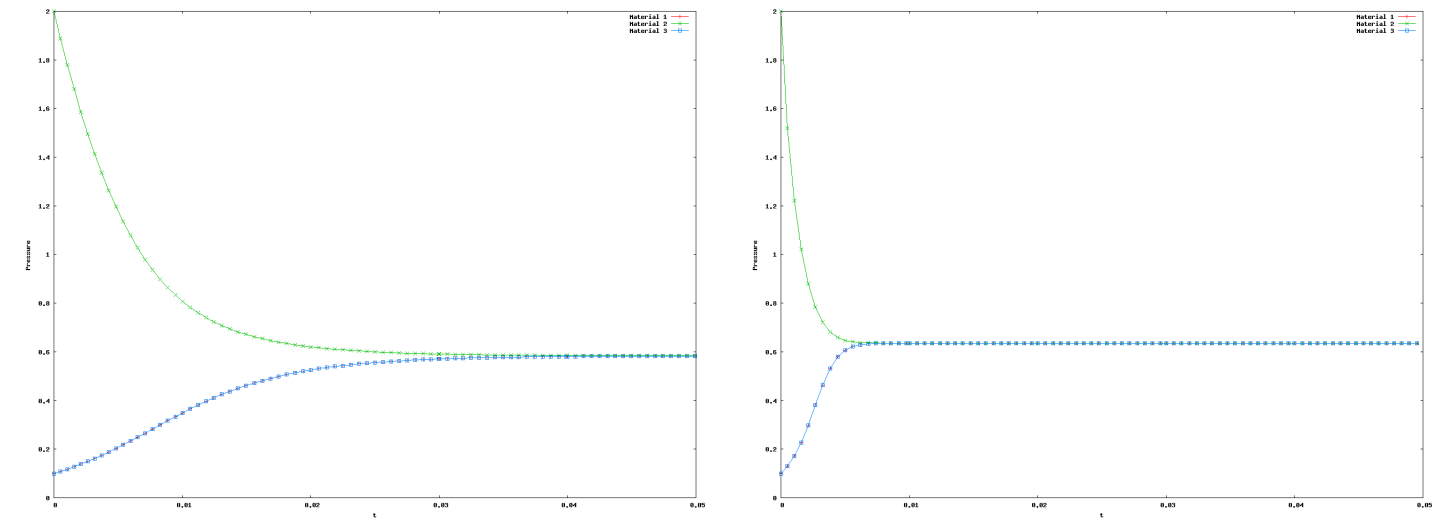

Fig. 41. Time histories of pressure, three-material one-cell - LHL. Left: Tipton, right: IA-SSD.

The pressure evolutions of the three materials for the LHL case are shown in Figure 41 for the Tipton and IA-SSD models. Because of the symmetry in the LHL case, the plots for the left and right materials coincide with each other for both models. 

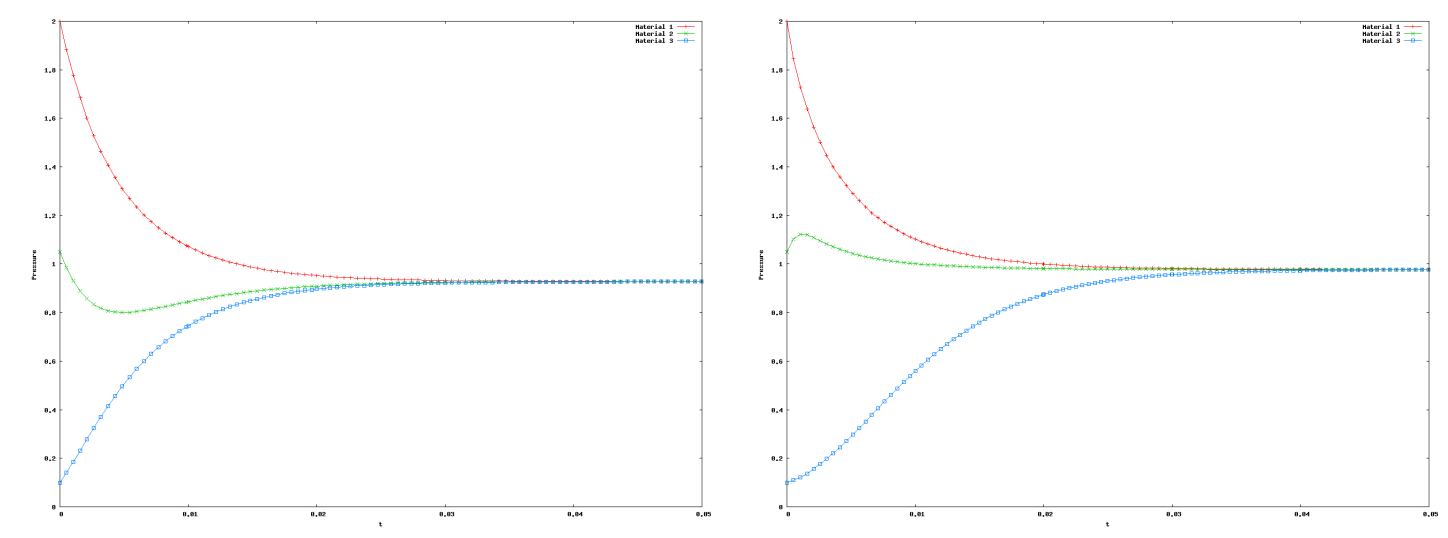

Fig. 42. Time histories of pressure, three-material one-cell - HML. Left: Tipton, right: IA-SSD.

$\underline{H M L}$ case

In this case the materials in the cell are configured as high-medium-low.

The pressure evolutions of the three materials for the HML case are shown in Figure 42 for the Tipton and IA-SSD models. In this case, the equilibration process for the IA-SSD model is more physical because the initial pressure in the middle material is close to the equilibrium pressure and the sub-scale dynamics allows both interfaces of the middle material to move simultaneously such that pressure in the middle cell changes only slightly. The reason for this is that in the IA-SSD model, the dynamics of a particular interface is driven by the difference of the material pressures related to the materials on both sides of the interface; in the Tipton model, the sub-scale dynamics of all interfaces is driven by the difference of the material pressure and the common pressure.

\subsection{Two-dimensional Lagrangian calculations}

This section presents results of two-dimensional Lagrangian calculations featuring multimaterial cells. In all calculations, the recently developed improved symmetric moment-of-fluid interface reconstruction method, [25], is used.

\subsubsection{Two-dimensional sanity checks}

\section{Two-material random volume fraction shock interaction}

A shock interaction test is performed over the computational domain $x=$ $[-1: 1], y=[0: 0.5]$. The left boundary is a piston with velocity $u=0.620174$ which generates a shock. The right boundary is a wall. The initial conditions are $\rho=1.0, p=1.0$ and velocity is zero for all internal nodes. Both materials 


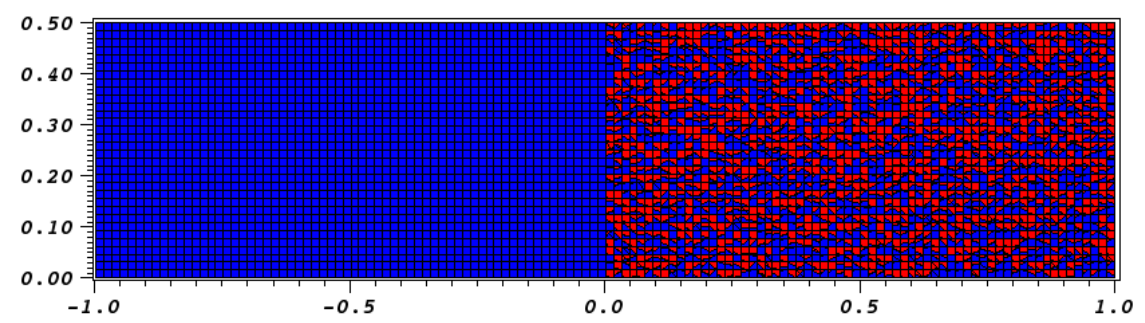

Fig. 43. Initial pseudo two-material random interface shock interaction mesh. The cells in the range $[-1: 0]$ are pure cells, the cells in the range $[0: 1]$ contain a fake contact, with material \# 1 shown in blue, and material \# 2 shown in red.
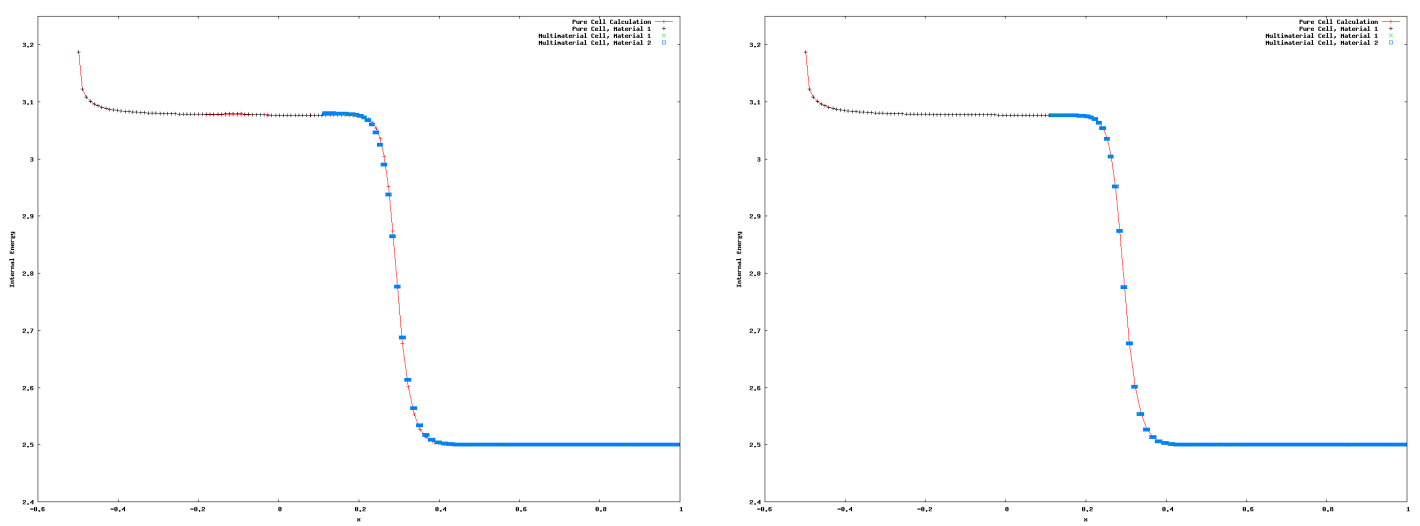

Fig. 44. Internal energy profiles for the pseudo two-material random volume fraction shock interaction. Left: Tipton, right: IA-SSD.

are the same ideal gas with $\gamma=1.4$. The initial mesh consists of $128 \times 32$ square cells. The simulation is run until $t=0.8$.

The shock wave forms in pure cells located in the range $[-1: 0]$, before interacting with multimaterial cells located in the range $[0: 1]$. The multimaterial cells contain two-materials which have identical properties with a randomly generated volume fraction. The interface reconstruction therefore produces randomly oriented interfaces. The initial material distribution is shown in Figure 43 with each cell constituent colored by the material.

The Tipton and IA-SSD multimaterial cell calculations are compared against the pure cell calculation. Figure 44 shows the internal energy profiles (all cells) obtained by each method at $t=0.8$. A magnified view of the internal energy profiles is shown in Figure 45. It can be seen that the Tipton solution introduces an error in both the pure and multimaterial cells. The IA-SSD calculation strongly matches the pure cell calculation in all cells.

This result is consistent with the corresponding one-dimensional calculations presented in Fig. 6. However, in contrast with the one-dimensional calculations where there is only one multimaterial cell, in two-dimensions there are many 

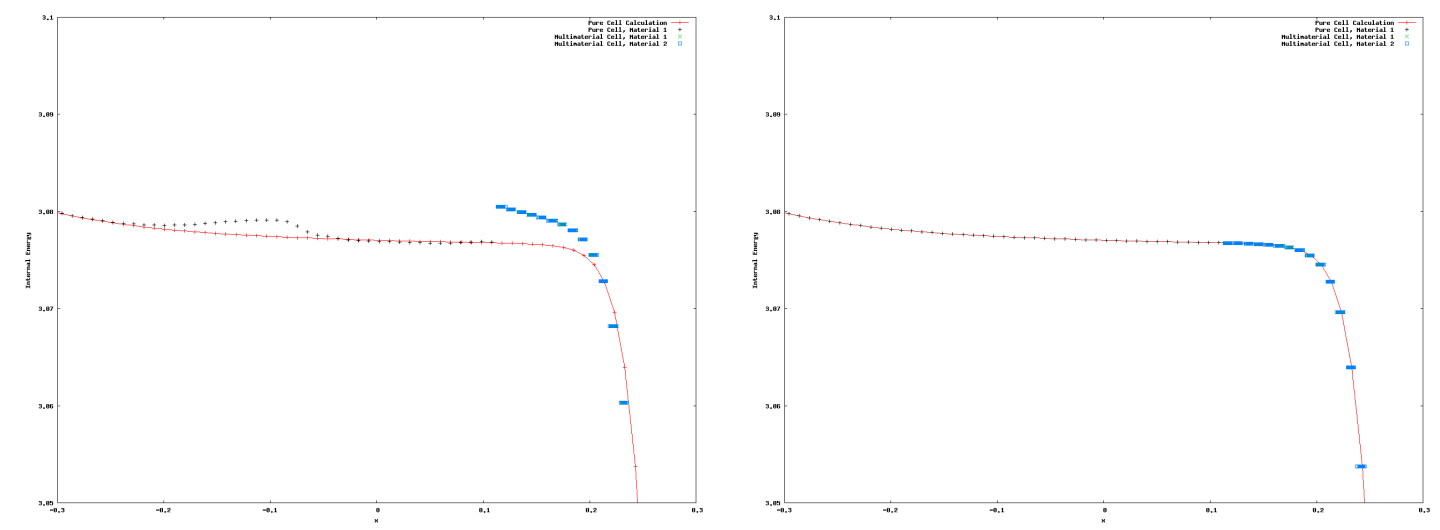

Fig. 45. Magnified views of the internal energy profiles for the pseudo two-material random volume fraction shock interaction. Left: Tipton, right: IA-SSD.
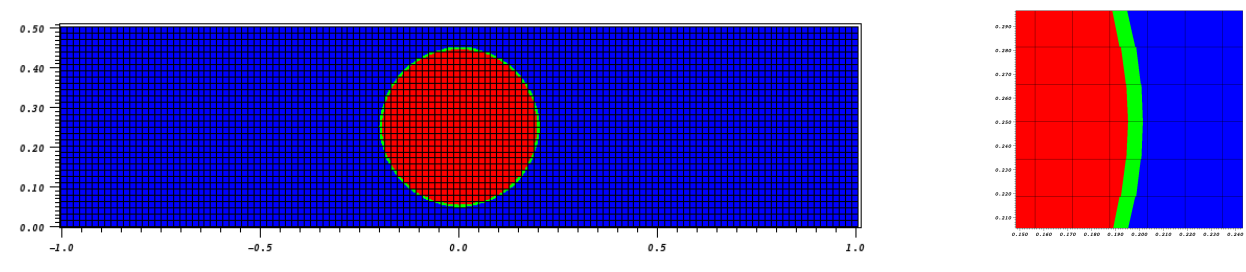

Fig. 46. Initial pseudo three-material ring shock interaction mesh - overview and magnified view of interface. Material \# 1 is shown in blue, material \# 2 in green, and material \# 3 in red.

multimaterial cells with different volume fractions and therefore the overshoots in internal energy have different magnitudes in the different multimaterial cells.

\section{Three-material ring shock interaction}

The shock interaction test is again performed featuring fake contacts between three materials defined by concentric rings enclosed by an additional material - all three materials have identical properties as in the previous example. The rings are centered at $x=0.0, y=0.25$, with radii $r=0.195$ and $r=0.2$. The initial material distribution is shown in Figure 46 with each cell constituent colored by the material number. Note that there are numerous formally three material cells.

Again, the Tipton and IA-SSD multimaterial cell calculations are compared against a pure cell calculation. Figure 47 shows the internal energy profiles (all cells) obtained by each method at $t=0.8$. A magnified view of the internal energy profiles is shown in Figure 48. It can be seen that for the Tipton model, values vary greatly in both the pure and two- and three-material cells defined by the ring. The IA-SSD calculation strongly matches the pure cell calculation in all cells. 

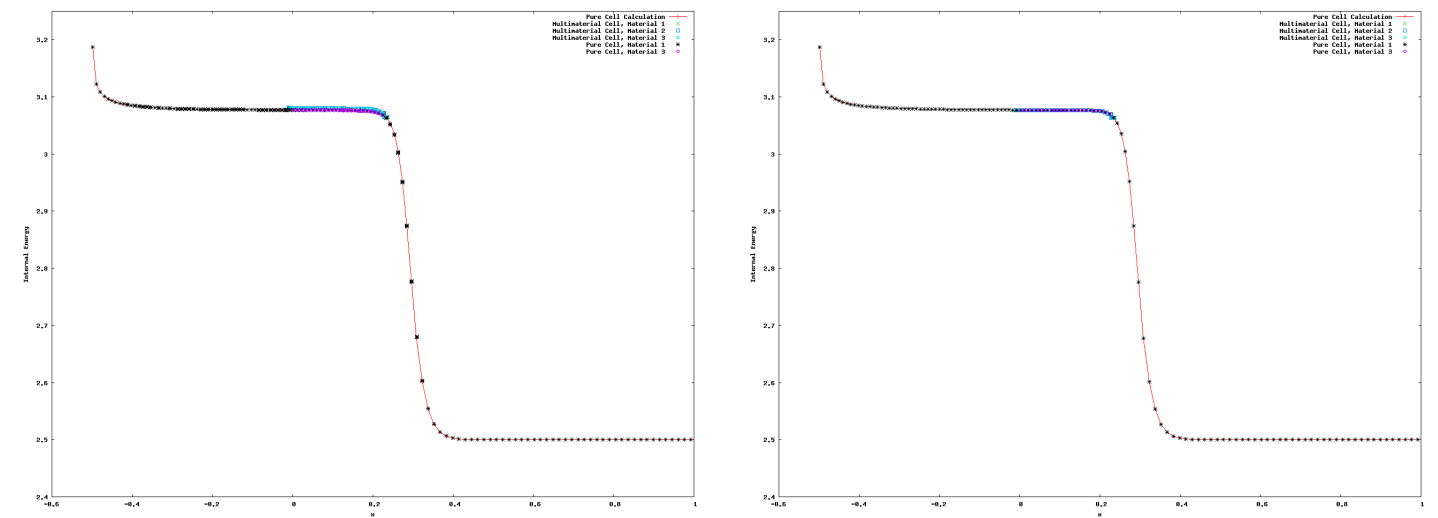

Fig. 47. Internal energy profiles for the pseudo three-material ring shock interaction. Left: Tipton, right: IA-SSD.
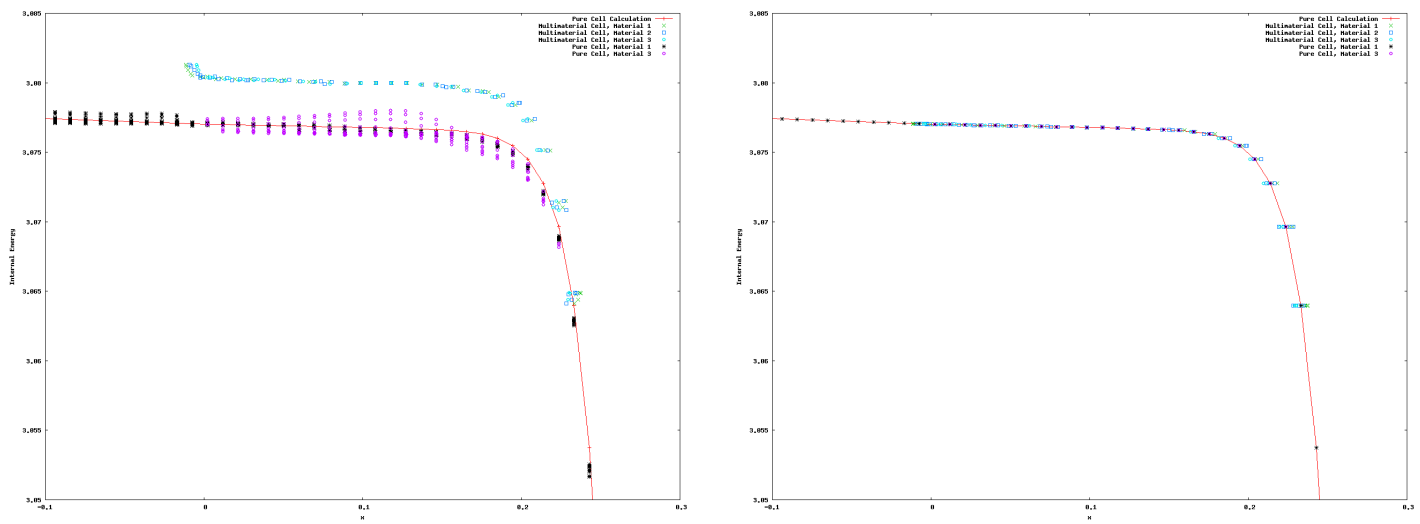

Fig. 48. Magnified views of the internal energy profiles for the pseudo three-material ring shock interaction. Left: Tipton, right: IA-SSD.

Figure 49 shows the computational meshes at $t=0.8$, colored by material internal energy for both the Tipton and IA-SSD calculations, with Figure 50 showing magnified views of the material interfaces. It can be seen that the material interfaces for both models are very similar for this problem.

The two-dimensional sanity checks support the conclusions of the one-dimensional calculations - Tipton's model creates artificial overshoots in the internal energy.

\subsubsection{Modified Sod problem. Three material cell with T-junction and symme- try preservation}

The two material modified Sod shock tube problem described in Section 8.1.3 is now cast as a formally three material problem. Initially the materials are arranged symmetrically, as shown in Fig. 51. The blue material on the left (material \# 1) corresponds to the left material in the statement of the two material Sod problem from Section 8.1.3. The green (material \# 2) and red 

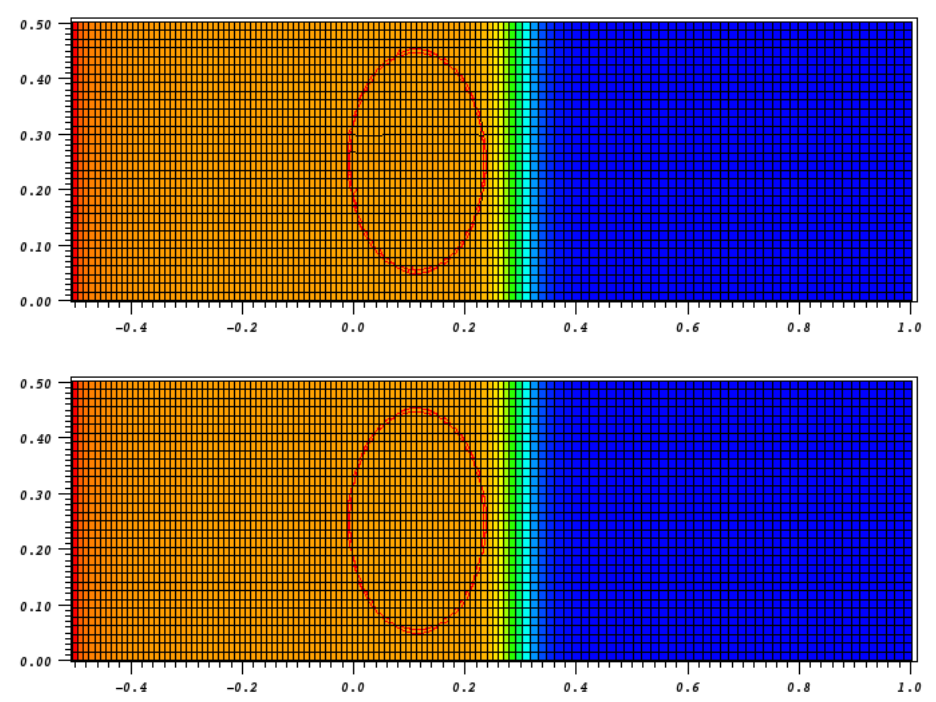

Fig. 49. Final pseudo three-material ring shock interaction mesh colored by material internal energy. The ring interfaces are shown in red. Top: Tipton, bottom: IA-SSD.
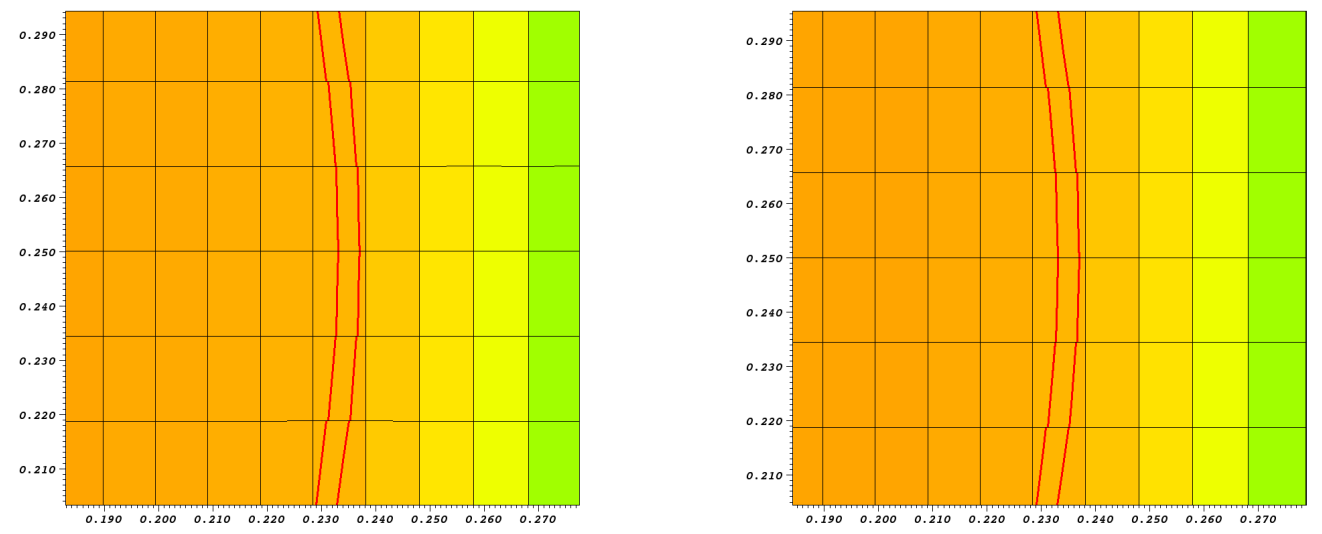

Fig. 50. Final pseudo three-material ring shock interaction mesh colored by material internal energy - magnified view of the interfaces, shown in red. Left: Tipton, right: IA-SSD.

(material \# 3) materials are formally different but have identical equations of state and identical initial parameters, with values corresponding to the state of the right material in Section 8.1.3.

Initial values of the parameters are as in the two material Sod problem from Section 8.1.3: $\rho_{1}=1.0, p_{1}=2.0, \gamma_{1}=2.0, \rho_{2}=\rho_{3}=0.125, p_{2}=p_{3}=0.1, \gamma_{2}=$ $\gamma_{3}=1.4$. The initial velocity is zero for all nodes. The final time is $t=0.2$. The computational domain is $(x, y) \in[0: 1] \times[0: 0.2]$. The initial mesh is defined by $100 \times 20$ square cells.

To obtain a reference solution, a pure cell calculation (using three materi- 


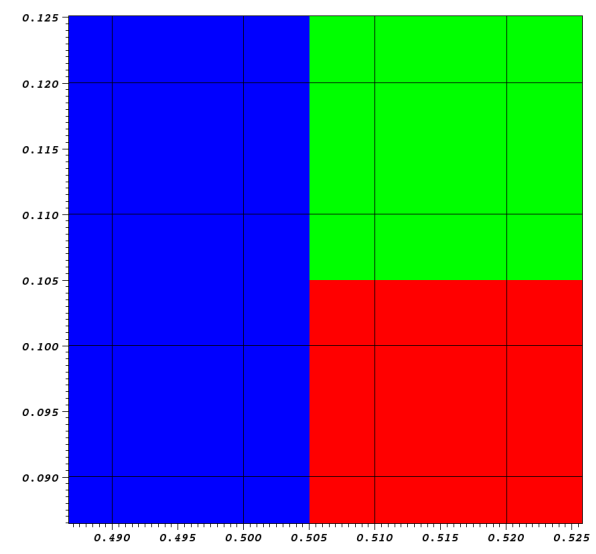

Fig. 51. Initial arrangements of the materials for modified Sod shock tube problem; symmetric ' $\mathrm{T}$ '-junction configuration - magnified view.

als) is also performed with $x^{\text {int }}=0.5, y^{\text {int }}=0.1(x>0.5)$. Multimaterial cell calculations are performed by shifting the interface positions to $x^{\text {int }}=$ $0.505, y^{i n t}=0.105(x>0.505)$. The shifted interface positions create a single three-material cell featuring a symmetric ' $\mathrm{T}$ ' junction topology with $\alpha_{1}=$ $0.5, \alpha_{2}=0.25, \alpha_{3}=0.25$, and additional multiple two-material cells along each interface with equal volume fractions.

The pure cell calculation reaches pressure equilibrium at the interface with $p_{1}=0.430363909693338$ and $p_{2}=0.430340672419380$. Additional information for the pure cell calculation is not presented due to similarities with the $1 \mathrm{D}$ calculation.

The Tipton and IA-SSD solutions both reach pressure equilibration in the multimaterial cells. When the Tipton closure model is used, the three-material cell equilibrates to $p=0.429935810731967$ with volume fraction $\alpha_{1}=0.840335797194088$, and the two-material neighbor located immediately above equilibrating to $p=0.430007706354197$ (coinciding with the three-material cell to 1 decimal place) with $\alpha_{1}=0.840277858149364$ (coinciding to 3 decimal places). When the IA-SSD closure model is used, the three-material cell equilibrates to $p=0.430160664438097$ with volume fraction $\alpha_{1}=0.865397666517836$, and the two-material neighbor above equilibrating to $p=0.430160664438673$ (coinciding to 12 decimal places) with $\alpha_{1}=0.865397666517698$ (coinciding to 12 decimal places).

Therefore although Tipton reaches equilibrium in each multimaterial cell, there is no consistency between cells, which results in a perturbation of the material interface in the cells around the ' $\mathrm{T}$ ' junction. Magnified views of the interface positions at $t=0.2$ are shown in Figure 52. The Tipton equilibrium pressures also show a larger discrepancy to the pure cell equilibrium pressures 

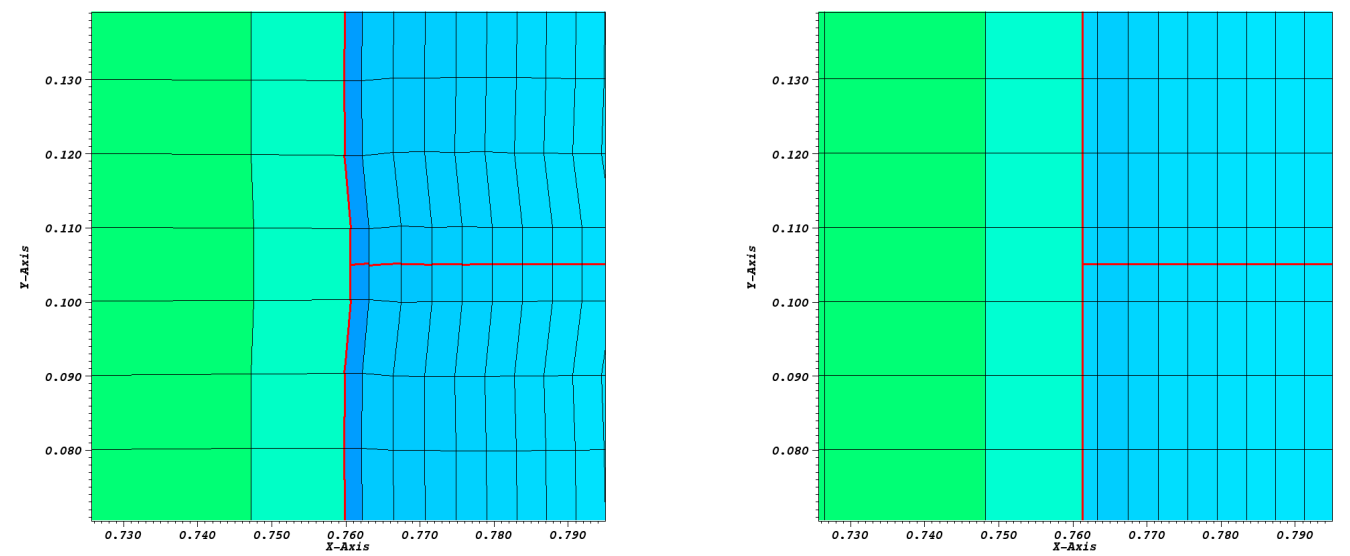

Fig. 52. Interface positions at $t=0.2$, modified Sod problem, 2D calculation, symmetric ' $\mathrm{T}$ '-junction case. Cells colored by material density, interface shown in red. Left: Tipton, right: IA-SSD.
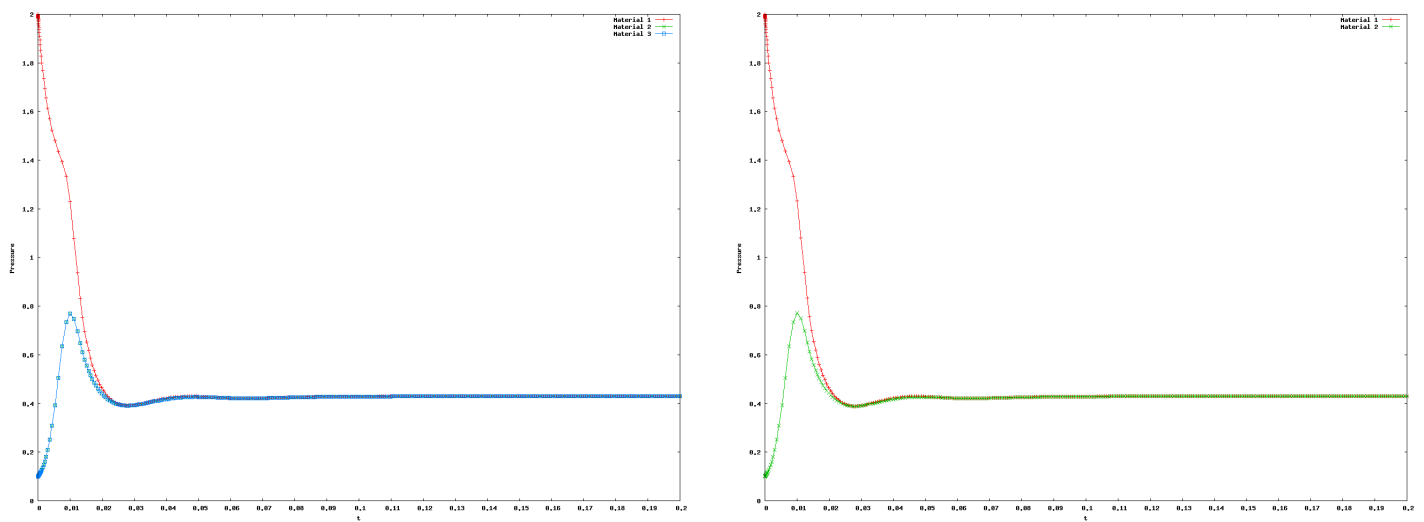

Fig. 53. Tipton time histories of pressures, three-material shock tube. Left: 3-material cell, right: 2-material cell.

compared to the IA-SSD-to-pure equilibrium pressures. It can be seen that the positions of interfaces for IA-SSD model remain symmetric.

Figures 53 and 54 show pressure evolutions for the Tipton and IA-SSD methods for the three material cell and the representative two material cell. The results presented in these figures are very similar to $1 \mathrm{D}$ case. Note that plots for materials \#2 and \#3 coincide.

In addition, a non-symmetric ' $\mathrm{T}$ ' junction case is considered. The interfaces are shifted to $x^{\text {int }}=0.5025, y^{\text {int }}=0.102(x>0.5025)$ so that a single threematerial cell featuring a non-symmetric ' $\mathrm{T}$ ' junction topology is created along with multiple non-symmetric two-material cells along each interface. The initial volume fractions in the three material cell are $\alpha_{1}=0.25, \alpha_{2}=0.6, \alpha_{3}=$ 0.15. A magnified view of the initial three-material cell is shown in Figure 55. 

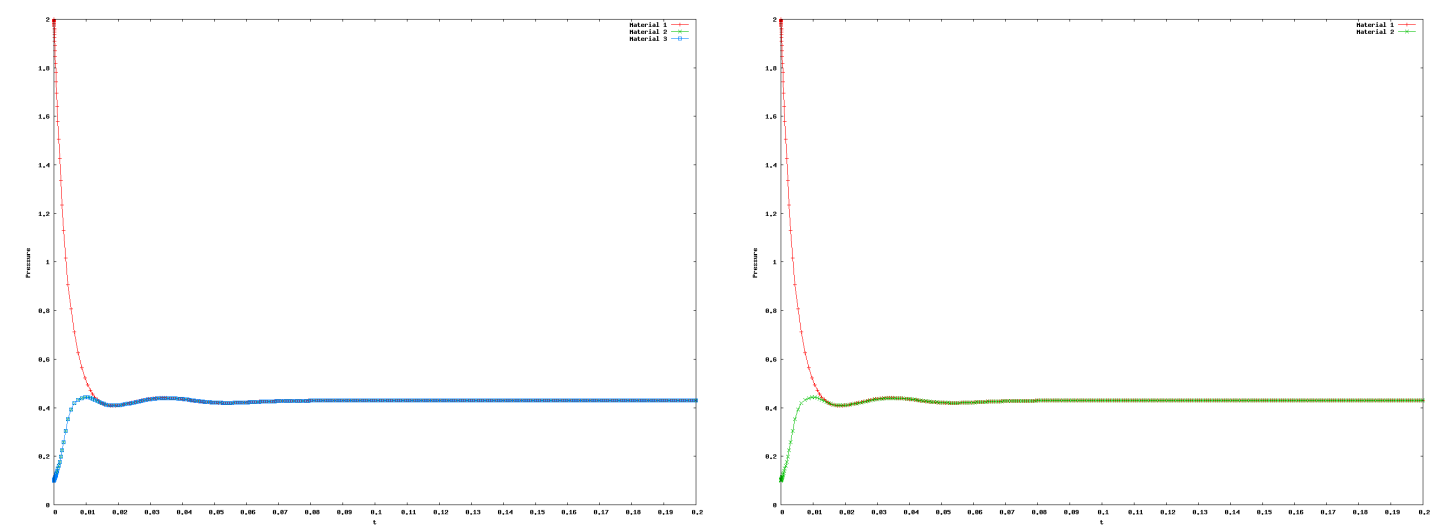

Fig. 54. IA-SSD time histories of pressures, three-material shock tube. Left: 3-material cell, right: 2-material cell.

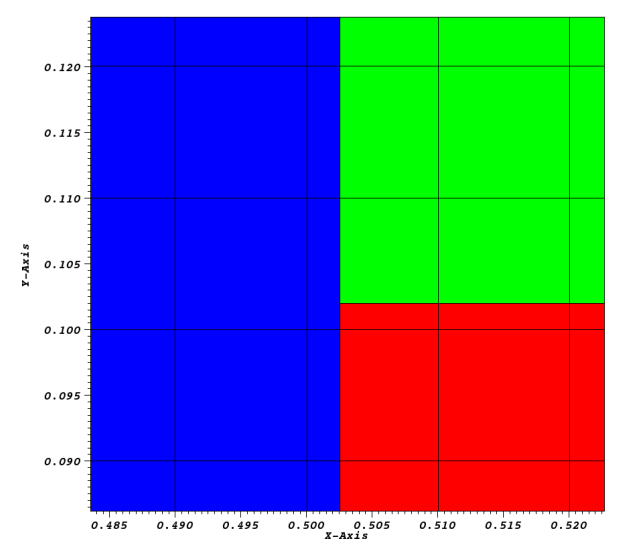

Fig. 55. Initial arrangement of the materials for the modified Sod shock tube problem; non-symmetric ' $T$ '-junction configuration - magnified view.

The pure cell calculation reaches pressure equilibrium at the interface with $p_{1}=0.430363909693338$ and $p_{2}=0.430340672419380$.

The Tipton and IA-SSD solutions again reach pressure equilibration in the multimaterial cells. When Tipton's closure model is used, the three-material cell equilibrates to $p=0.430218541175767$ with volume fraction $\alpha_{1}=0.688718110763616$, with the two-material neighbor above equilibrating to $p=0.430280668274608$ (coinciding with the three-material cell to 4 decimal place) with $\alpha_{1}=0.688593278552696$ (coinciding to 3 decimal places). When the IA-SSD closure model is used, the three-material cell equilibrates to $p=0.430326160653562$ with volume fraction $\alpha_{1}=0.712819633884100$, with the two-material neighbor above equilibrating to $p=0.430326160653568$ (coinciding to 14 decimal places) with $\alpha_{1}=0.712819633884125$ (coinciding to 13 decimal places). Again, although Tipton reaches equilibrium in each multimaterial cell, there is no consistency between cells. Also, we believe that the sub-scale dynamics for the three material cell for Tipton's model is not correct because it does not take into account 

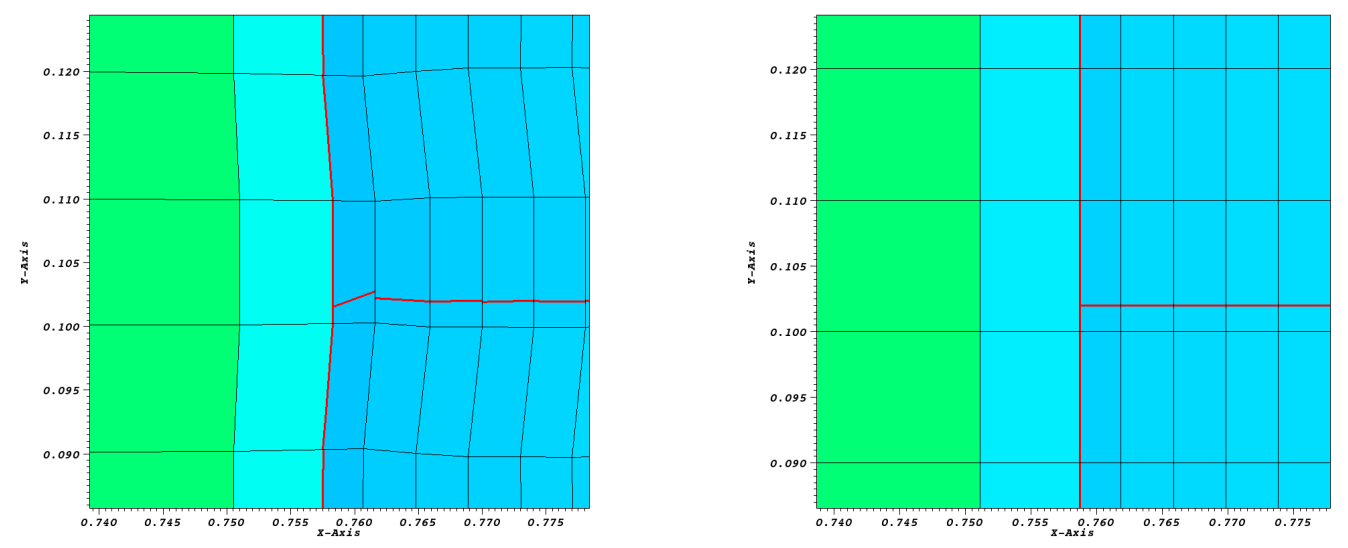

Fig. 56. Interface positions at $t=0.2$, modified Sod problem, 2D calculation, non-symmetric ' $\mathrm{T}$ '-junction case. Cells colored by material density, interface shown in red. Left: Tipton, right: IA-SSD.

the interface area between materials explicitly. The dynamics of each interface is also driven with respect to the common pressure, and not in a pair-wise fashion between materials. These factors result in the perturbation of the material interface in the two- and three-material cells around the ' $\mathrm{T}$ ' junction. Magnified views of the interface positions at $t=0.2$ are shown in Figure 56 . The interfaces for Tipton's model are visibly perturbed, whereas the positions of interfaces for the IA-SSD model remain correct.

\subsection{Two-dimensional arbitrary Lagrangian-Eulerian calculations}

In this Section results are presented for multimaterial ALE calculations. The remapping stage of the multimaterial ALE scheme is described in [49].

\subsubsection{Impact test}

Hydrocodes are often required to simulate high deformation and high strain rate problems involving high speed impacts between solid materials. In many situations an impactor is launched at high speed via explosives or a gas gun and driven into another target material to generate a strong shock in the target material. These problems lead to high material deformation and require the introduction of multimaterial cells and either Eulerian or ALE techniques to run to completion. The robustness and accuracy of these simulations will both be influenced by the quality of the closure model. A simplified purely hydrodynamic test problem has been developed using ideal gas equations of state, which is representative of such impact problems in an attempt to assess whether the new IA-SSD closure model will offer benefits for such challenging 


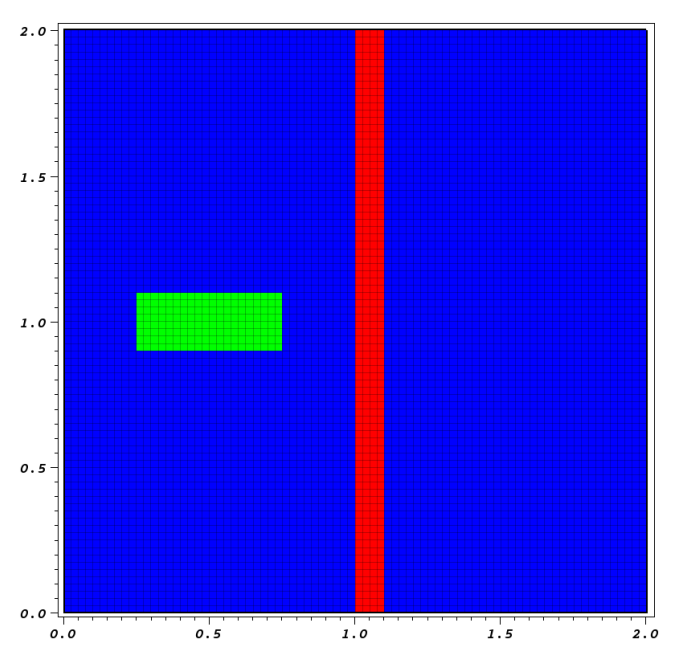

Fig. 57. Initial topology for the impact test, cells colored by material number.

simulations.

An impact test is performed consisting of a volume of high density, low compressibility gas traveling through air towards a thin layer of medium density gas. The high density gas volume collides with the medium gas resulting in a large amount of deformation of both materials and fragments (featuring very small volume fractions) breaking free.

The initial configuration of the materials is shown in Figure 57, colored by material number.

The test is performed in the domain $[0,2] \times[0,2]$, with the high density gas in the range $0.25 \leq x \leq 0.75,0.9 \leq y \leq 1.1$ (green material in Fig. 57, the medium density gas in the range $1 \leq x \leq 1.1$ (red material) and air elsewhere (blue material). The air has thermodynamic properties $\rho_{1}=1.0, p_{1}=1.0, \gamma_{1}=$ $1.4, u=0$ and $v=0$, the high density gas has properties $\rho_{2}=20.0, p_{2}=$ 2.0, $\gamma_{2}=50.0, u=0.2$ and $v=0$, and the medium density gas has properties $\rho_{3}=15.0, p_{3}=1.0, \gamma_{3}=5 / 3, u=0$ and $v=0$. The simulation is run until $t=$ 8.0 with $80 \times 80$ cells. All cells initially contain only one material, however due to the velocity of the high density gas (impactor) and resulting deformation, simulations must be performed in the ALE10 regime ${ }^{10}$ with a rezone to a relaxed mesh (defined by a single iteration of the Winslow algorithm to retain the Lagrangian mesh as much as possible) so that numerous multimaterial cells are created. This test allows an examination of the robustness of the IASSD and Tipton closure models for the case when materials have significantly different properties.

$\overline{10}$ Ten Lagrangian steps followed by one ALE step. 


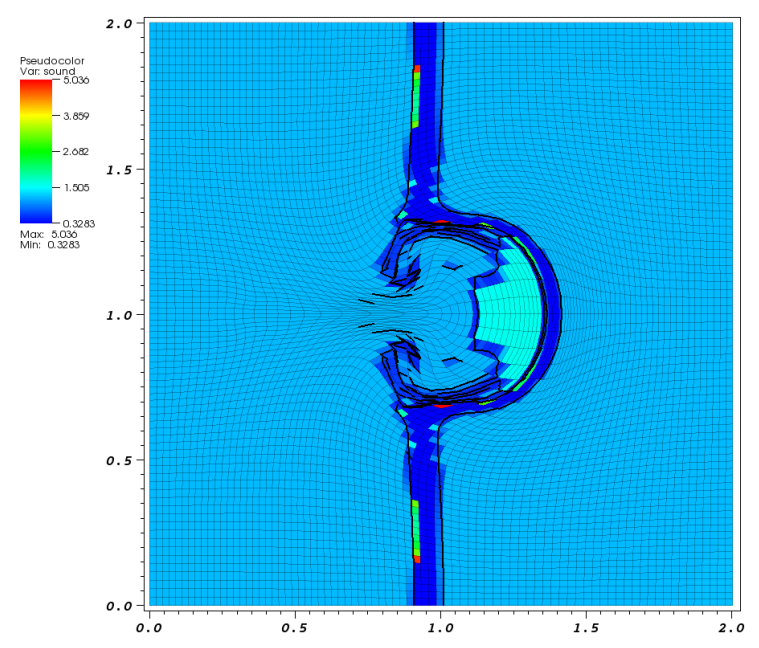

Fig. 58. Cell sound speed values for the Tipton simulation of the impact test case at $t=4.4705$ (just prior to simulation failure).

A significant result of this test case is the failure of the Tipton calculation at $t=4.4705$ due to degeneration of the time step. At this stage the mesh has undergone a reasonably large amount of deformation and fragments with small volume fractions have been ejected. The sound speed in all cells prior to the failure are shown in Figure 58, where isolated extreme values are visible (a maximum value $c=105.52$ is recorded at $t=3.996$ ).

Such values of the sound speed contribute to the degenerating time step and tangling of the mesh, and are not seen in the IA-SSD simulation. Figure 59 shows the values of sound speed at $t=3.0$ for both the Tipton and IA-SSD calculations. In this comparison it can be seen that the IA-SSD simulation does not introduce extreme values of cell sound speed in the multimaterial cells.

It may also be noted that while the general behavior of the two simulations tends to appear similar, significant differences between the two solutions are apparent. Figure 60 shows magnified views of the material pressure distributions around the collision zone at $t=4.25$. The pressure of the air compressed between the high- and medium-density materials differs greatly between methods. The IA-SSD approach achieves equilibrium in all materials, whereas the Tipton solution results in a low pressure for the air. Additionally, the Tipton pressure at the vortices following the impact also differs from the IA-SSD pressures in the same locations.

The improved robustness afforded by the IA-SSD approach allows the simulation to run until completion at $t=8$, as shown in Figure 61. 

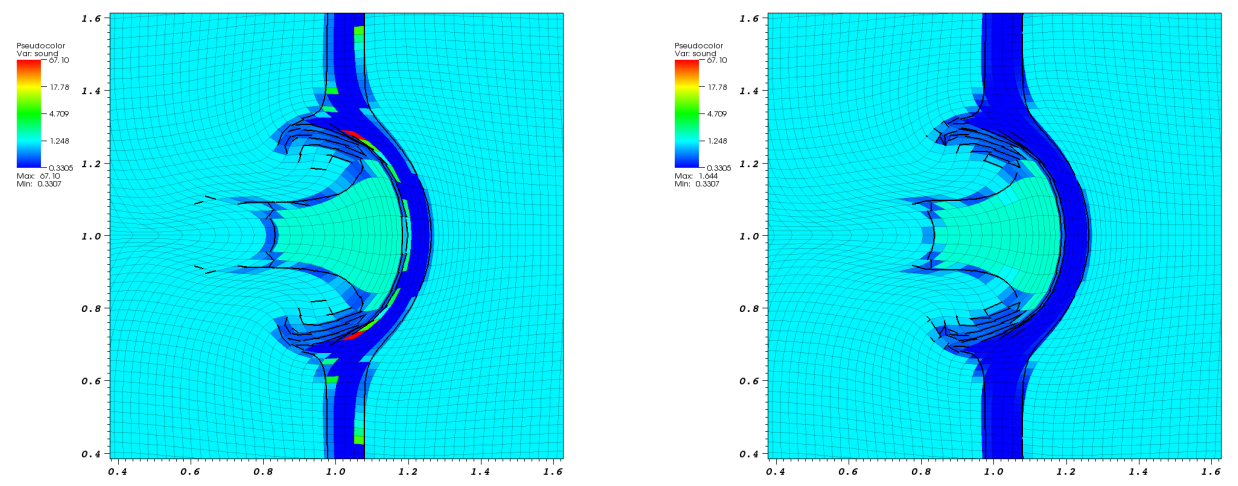

Fig. 59. Sound speed values (logarithm scale) for the impact test case at $t=3.0$. Left: Tipton cell values, right: IA-SSD cell values. Note also that the IA-SSD simulation shows less initial break up of material, possibly due to the improved material centroid update scheme.
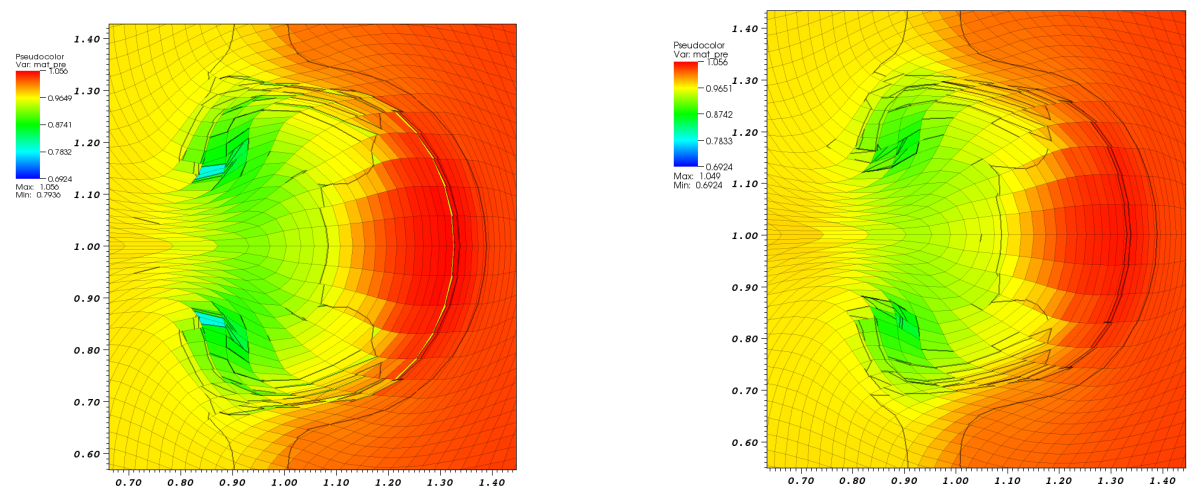

Fig. 60. Material pressure values for the impact test case at $t=4.25$. Left: Tipton, right: IA-SSD.

\section{Conclusions}

In this paper, a systematic description of the new interface-aware sub-scaledynamics (IA-SSD) closure model for the Lagrangian stage of multimaterial arbitrary Lagrangian-Eulerian methods has been presented. The IA-SSD closure model consists of two stages. During the first, bulk, stage, the well known equal compressibility model is used. During the second stage, sub-scale interactions of the materials inside the multimaterial cell are taken into account. At this point, information about the topology of the materials inside the multimaterial cell is utilized, which allows the inclusion of the orientations of internal interfaces in the model. Each material interacts in a pair-wise fashion with the materials with which it has a common boundary. The interactions are based on the solution of the acoustic Riemann problem between each pair 

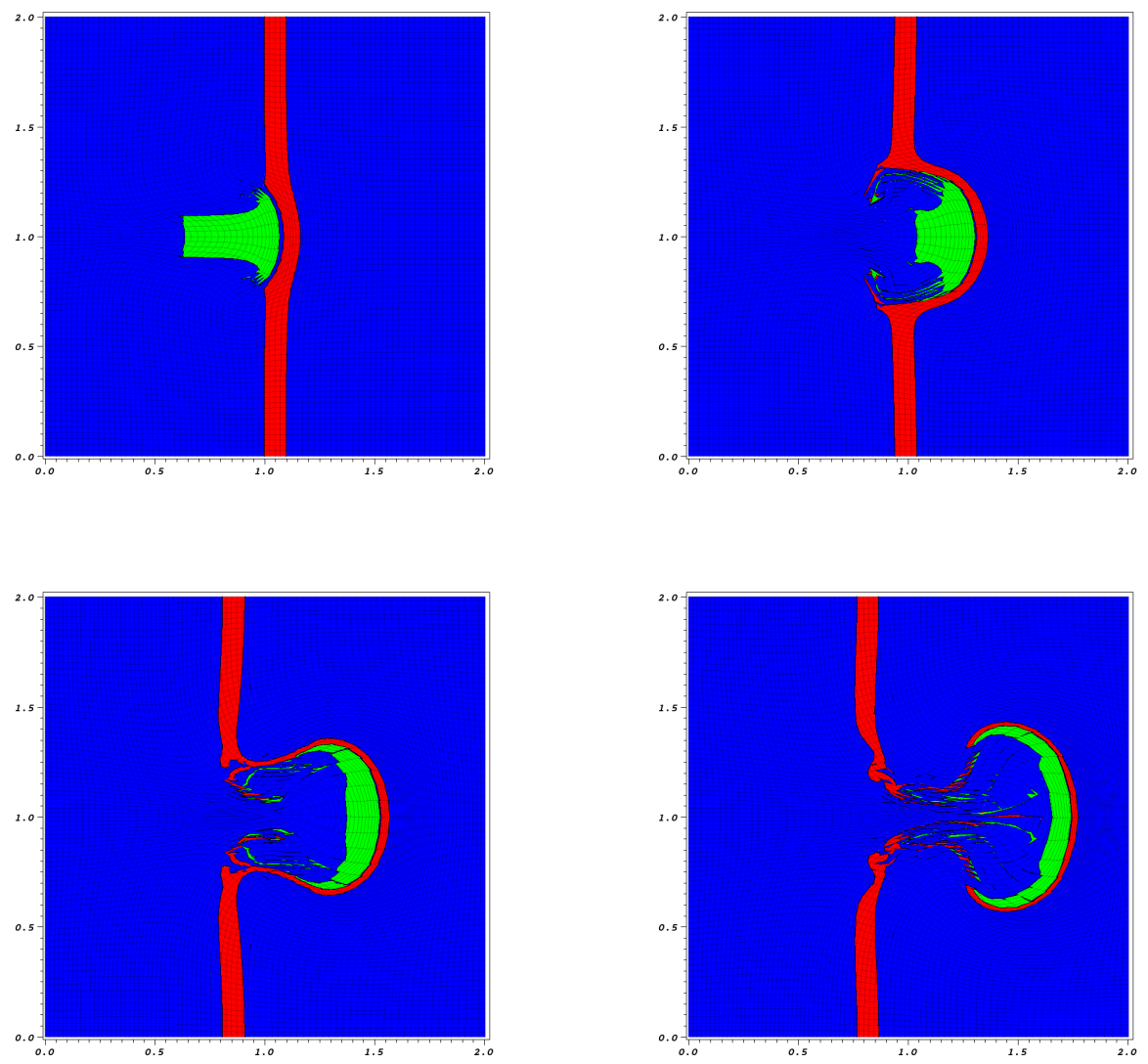

Fig. 61. Topology of the impact test at four stages of the IA-SSD simulation, cells colored by material number. Top left: simulation at $t=2.0$; top right, $t=4.0$; bottom left, $t=6.0$, bottom right, $t=8.0$.

of materials and is limited using physically justified constraints. In this paper, three types of constraints are used: positivity of volume, positivity of internal energy and controlling the rate of pressure relaxation.

To determine values for the limiters, a constrained-optimization framework is employed using a quadratic objective function with linear constraints. The optimization problem is solved for each multimaterial cell; in the case of two materials, this problem has an explicit solution. The new scheme is described as a "framework" because the theory for the optimal choice of parameters in the constraints is yet to be developed. However, the algorithm guarantees the positivity of the material volume and internal energy as well as the smooth relaxation of the pressure - this allows a significant increase in the robustness of the overall ALE algorithm.

Details of how to include the IA-SSD closure model into the staggered discretization of the Lagrangian stage of the ALE method have been provided. The overall algorithm is conservative, and in particular, momentum and total 
energy are preserved. One of the features of the discretization is the exchange of internal energies between materials in the multimaterial cell.

The results of comprehensive testing of the new model have been presented for one- and two-dimensional multimaterial Lagrangian hydrodynamics along with representative results for 2D multimaterial arbitrary Lagrangian-Eulerian calculations. The numerical tests have shown that in most cases the new IASSD closure model produces better results compared to the well known Tipton closure model. In particular, the IA-SSD model better preserves spatial symmetries by taking into account the orientation of the material interfaces, and performing sub-scale interactions on a pair-wise basis. The numerical results show that the use of limiters during the sub-scale stage allows the robustness of the overall ALE algorithm to be improved.

It is believed that the sub-scale dynamics approach will allow the inclusion of additional physics in the closure model. In the near future, further improvements to the bulk phase are planned, which will include development of the multiphase artificial viscosity, subzonal pressures and hourglass forces. We believe that it may be vitally important when there are large differences in material properties at an interface. The correct partitioning of the work done by such additional forces amongst the materials will put the subcell dynamics stage of our method in a much better starting position.

An extension to the IA-SSD framework is also planned for the case where a multimaterial cell may contain voids, and in particular, an algorithm which will allow void closure is considered. Also planned is the development of a sub-scale closure model which will include the treatment of solids and slide lines. Finally, the theory regarding the optimal choice of parameters used in the constraints will be developed along with the possibility of the introduction of other physically justified constraints.

\section{Acknowledgments}

This work was performed under the auspices of the National Nuclear Security Administration of the US Department of Energy at Los Alamos National Laboratory under Contract No. DE-AC52-06NA25396. The authors gratefully acknowledge the partial support of the US Department of Energy Office of Science Advanced Scientific Computing Research (ASCR) Program in Applied Mathematics Research and the partial support of the US Department of Energy National Nuclear Security Administration Advanced Simulation and Computing (ASC) Program.

The authors thank D. Burton. A. Harrison, J. Fung, T. Canfield, J. Kamm, M. 
François, D. Youngs, D. Benson, Y. Yanilkin, Y. Bondarenko, B. Wendroff, J. Grove, B. Després, R. Loubère, P. Vachal, M. Kuchařík, T. Masser, E. Dendy, B. Rider, D. Miller, R. Abgrall for many useful discussion over the years.

\section{References}

[1] C. Hirt, A. Amsden, J. J. L. Cook, An arbitrary Lagrangian- Eulerian computing method for all flow speeds, Journal of Computational Physics 14 (3) (1974) 227-253.

[2] J. Donea, S. Giuliani, J. P. Halleux, An arbitrary Lagrangian-Eulerian finite element methods for transient dynamic fluid structure interactions, Com. Meth. Appl. Mech. Eng., 33, (1982) 689-723.

[3] D. Benson, Computational methods in Lagrangian and Eulerian hydrocodes, Journal Computer Methods in Applied Mechanics and Engineering 99 (2-3) (1992) 235-394.

[4] R. Tipton, CALE mixed zone pressure relaxation, Tech. rep., Lawrence Livermore National Laboratory - Private communication (1989).

[5] D. Miller, G. Zimmerman, An algorithm for time evolving volume fractions in mixed zones in Lagrangian hydrodynamics calculations, Tech. rep., Lawrence Livermore National Laboratory, UCRL-PRES-223908 (2006).

[6] M. Baer, J. Nunziato, A two-phase mixture theory for the deflagration-todetonation transition (ddt) in reactive granular materials, Int. J. Multiphase Flow 12 (1986) 861-889.

[7] A. Murrone, H. Guillard, A five equation reduced model for compressible two phase flow problems, Journal of Computational Physics 202 (2005) 664-698.

[8] J. Kamm, M. Shashkov, J. Fung, A. Harrison, T. Canfield, A comparative study of various pressure relaxation closure models for one-dimensional twomaterial Lagrangian hydrodynamics, Int. J. Numer. Meth. Fluids 65 (11-12) (2010) 1311-1324.

[9] M. Shashkov, Closure models for multimaterial cells in arbitrary LagrangianEulerian hydrocodes, Int. J. Numer. Meth. Fluids 56 (2007) 1497-1504.

[10] M. François, M. Shashkov, E. Dendy, R. Lowrie, A comparative study of staggered and Lagrangian formulation for multimaterial hydrodynamics, 2010, proceedings of the Nuclear Explosives Code Developers' Conference (NECDC).

[11] M. François, M. Shashkov, E. Dendy, R. Lowrie, Mixture models for multimaterial Eulerian and Lagrangian hydrocodes, Tech. rep., presentation at 8th International Conference on New Models and Hydrocodes for Shock Wave Processes - Paris, France; Available as Los Alamos National Laboratory Report LAUR-10-03391 at http://cnls. lanl.gov/ shashkov (2010). 
[12] Y. Yanilkin, E. Goncharov, V. Kolobyanin, V. Sadchikov, J. Kamm, M. Shashkov, W. Rider, Multi-material pressure relaxation methods for Lagrangian hydrodynamics, Computers \& Fluids 83 (2013) 137-143.

[13] Y. Bondarenko, Y. Yanilkin, Computation of the thermodynamic parameters in the mixed cells in gas dynamics, Mathematical Modeling 14 (2002) 63-81.

[14] V. Delov, V. Sadchikov, Comparison of several models for computation of thermodynamics parameters in heterogeneous Lagrangian cells, VANT, Mathematical Modeling of Physical Processes 1 (2005) 57-70.

[15] E. Goncharov, Y. Yanilkin, New method for computation of thermodynamical states of the materials in the mixed cells, VANT, Mathematical Modeling of Physical Processes 3 (2004) 16-30.

[16] A. Barlow, A new Lagrangian scheme for multimaterial cells, in: Proceedings of European Congress on Computational Methods in Applied Sciences and Engineering. ECCOMAS Computational Fluid Dynamics Conference, 2001, pp. $235-294$.

[17] B. Després, F. Lagoutière, Numerical solution of two-component compressible fluid model with interfaces, Progress in Computational Fluid Dynamics 7 (2007) 295-310.

[18] R. N. Hill, A. Barlow, M. Shashkov, Interface-aware sub-scale dynamics closure model, Tech. Rep. LA-UR-12-21959, International Conference on Numerical Methods in Multiphase Flows ; 2012-06-12 - 2012-06-14 ; State College, Pennsylvania, United States and 3rd International EULAG Workshop on Eulerian/Lagrangian methods for fluids. 25-28 June, 2012, Loughborough UK.

[19] A. Barlow, R. Hill, M. Shashkov, Interface-aware sub-scale dynamics closure model for multimaterial cells in Lagrangian gas dynamics, Tech. rep., Los Alamos National Laboratory Report LA-UR -12-00980 (2012).

[20] R. N. Hill, A. Barlow, M. Shashkov, Interface-aware sub-scale dynamics closure model, Tech. Rep. LA-UR-12-24964, Nuclear Explosive Code Development Conference; Livermore, California, United States, 2012.

[21] J. W. Grove, Pressure-velocity equilibrium hydrodynamic models, Acta Mathematica Scientia 30B(2) (2010) 563-594.

[22] A. Harrison, M. Shashkov, J. Fung, J. Kamm, T. Canfield, Development of a sub-scale dynamics model for pressure relaxation of multi-material cells in Lagrangian hydrodynamics, Tech. rep., Proceedings of the conference "New Models and Hydrocodes for Shock Wave Processes in Condensed Matter", Paris, France, May 24-28,2010, EPJ Web of Conferences, available as Los Alamos National Laboratory Report LAUR-10-06964 at http://cnls.lanl. gov/ shashkov (2010).

[23] V. Dyadechko, M. Shashkov, Reconstruction of mutli-material interfaces from moment data, J. Comput. Phys. 227 (2008) 5361-5384. 
[24] H. Ahn, M. Shashkov, Multi-material interface reconstruction on generalized polyhedral meshes, J. Comput. Phys. 226 (2007) 2096-2132.

[25] R. Hill, M. Shashkov, The symmetric moment-of-fluid interface reconstruction algorithm, Journal of Computational Physics 249 (2013) 180-184.

[26] E. Caramana, D. Burton, J. Shashkov, M, P. Whalen, The construction of compatible hydrodynamics algorithms utilizing conservation of total energy, J. Comput. Phys. 146 (1998) 227-262.

[27] H. Stewart, B. Wendroff, Two-phase flow: models and methods, Journal of Computational Physics 56 (1984) 363-409.

[28] A. Barlow, R. Hill, M. Shashkov, Constrained optimization framework for interface-aware sub-scale dynamics closure models for multimaterial cells in Lagrangian and arbitrary Lagrangian-Eulerian hydrodynamics, Tech. rep., Los Alamos National Laboratory, Los Alamos National Laboratory Report LAUR13-26180 (2013).

[29] D. Kuzmin, R. Löhner, S. Turek (Eds.), Flux-corrected Transport: Principles, Algorithms, and Applications, 2nd Edition, Springer, 2012.

[30] D. Burton, Multidimensional discretization of conservation laws for unstructured polyhedral grids, Tech. rep., Proceedings of the Second International Workshop on Analytical Methods and Process Optimization in Fluid and Gas Mechanics (VNIIEF, Sarov, Russia, 1994), available as UCRLJC-118306; CONF-9409314-1 at http://www.osti.gov (1994).

[31] J. Nocedal, S. Wright, Numerical Optimization, Springer, 1999.

[32] K. Schittowski, Q1: A Fortran code for convex quadratic programming - user's guide, Tech. rep., University of Bayreuth, Dept. of Computer Science, www. klaus-schittkowski.de/software.htm (2011).

[33] J. Zukas, Introduction to Hydrocodes, Studies in Applied Mechanics - 49, Elsevier, 2004.

[34] C. Anderson, J. Padraic, E. O'Donoghue, D. Skerhut, A mixture theory approach for the shock response of composite materials, Journal of Composite Materials 24 (1990) 1159-1178.

[35] R. Abgrall, S. Karni, Computations of compressible multifluids, J. Comput. Phys. 169 (2001) 594-623.

[36] M. Sun, A thermodynamic and dynamic subgrid closure model for two-material cells, Int. J. Numer. Meth. Fluids 73 (2) (2013) 130-151.

[37] S. T. Munkejord, M. Papin, The effect of the interfacial pressure in the discreteequation multiphase model, Computers \& Fluids 36 (2007) 742-757.

[38] S.-P. Wang, M. Anderson, J. Oakley, M. Corradini, R. Bonazza, A thermodynamically consistent and fully conservative treatment of contact discontinuities for compressible multicomponent flows, J. Comput. Phys. 195 (2004) 528-559. 
[39] R. Abgrall, R. Saurel, Discrete equations for physical and numerical compressible multiphase flows, J. Comput. Phys. 186 (2003) 361-396.

[40] R. Saurel, R. Abgrall, A multiphase Godunov methods for compressible multifluid and multiphase flows, J. Comput. Phys. 150 (1999) 425-467.

[41] D. Igra, M. Sun, Shock-water column interaction, from initial impact to fragmentation onset, AIAA, Journal 48 (2010) 2763-2771.

[42] A. Morin, T. Flatten, S. Numkerjord, Roe scheme for compressible six-equations two-fluid model, Int. J. Numer. Meth. Fluids 72 (4) (2012) 478-504.

[43] X. Hu, B. Khoo, An interface interaction method for compressible multifluids, J. Comput. Phys. 198 (2004) 35-64.

[44] W. Rider, E. Love, M. Wong, O. Strack, S. Petney, D. Labreche, Adaptive methods for multi-material ALE hydrodynamics, Int. J. Math. Fluids 65 (1112) (2011) 1325-1337.

[45] E. Caramana, M. Shashkov, P. Whalen, Formulations of artificial viscosity for multi-dimensional shock wave computations, J. Comput. Phys. 144 (1998) 7097.

[46] K. Lipnikov, G. Manzini, M. Shashkov, Mimetic finite difference method, J. Comput. Phys. 257 (Part-B) (2014) 1163-1228.

[47] M. Kuchařík, R. Garimella, S. Schofield, M. Shashkov, A comparative study of interface reconstruction methods for multi-material ALE simulations, J. Comput. Phys. 229 (2010) 2432-2452.

[48] J. Kamm, M. Shashkov, A pressure relaxation closure model for onedimensional, two-material Lagrangian hydrodynamics based on the Riemann problem, Commun. Comput. Phys. 7 (5) (2010) 927-976.

[49] M. Kuchařík, M. Shashkov, Conservative multi-material remap for staggered multi-material arbitrary Lagrangian-Eulerian methods, J. Comput. Phys. 258 (2014) 268-304.

[50] Ya.B. Zeldovich and Yu.P. Raizer, Physics of shock waves and high-temperature hydrodynamic phenomena, Dover Publications, INC. Mineola, New York, 2002. 